UNITED STATES DEPARTMENT OF THE INTERIOR

GEOLOGICAL SURVEY

\title{
ANALYTICAL DATA ON THE PHOSPHORIA FORMATION WESTERN UNITED STATES
}

By

Robert A. Gulbrandsen

Open-file report $75-554$

1975

This report is preliminary and has not been edited or. reviewed for conformity with Geological Survey standards and nomenclature 
Analytical Data on the Phosphoria Formation

by R. A. Gulbrandsen

Contents

Page

Introduction

1

Tables of Analytical Data

Table 1. Chemical composition of the quartz-silicate rocks of the Phosphoria Formation

2. Chemical composition of the carbonate rocks of the Phosphoria Formation

3. Mercury, thallium, and total nitrogen in samples of

the Meade Peak Member of the Phosphoria Formation

at Conda (lot 1200), Idaho

4. Mercury, thallium, and total nitrogen in samples of

the Meade Peak Member of the Phosphoria Formation at

Trail Canyon (lot 1206), Idaho

5. Chemical composition of samples from the vanadiferous zone, Bloomington Canyon (lot 1272), Idaho

6. Analyses of two samples of the vanadiferous zone at Coal

Canyon (1ot 1201), Wyoming

7. Vanadium analyses of the Phosphoria Formation at Bear

Creek (lot 1353), Idaho

8. Vanadium analyses of the Phosphoria Formation at Gravel Creek Divide (lot 1308), Idaho 


$$
\text { Tables of Analytical Data - Continued }
$$

9. Selenium analyses of parts of the Phosphoria Formation at 13 localities

10. Analytical data on Meade Peak Member of Phosphoria Formation at Snowdrift Mountain (lot 1372), Idaho

Figures

Figure 1. Location of analyzed samples of quartz-silicate rocks of table 1. Numbers are the analysis numbers - -2

2. Location of analyzed samples of carbonate rocks of table 2. Numbers are the analysis numbers

3. Location of analyzed samples of tables 3 to 10. Numbers are the lot numbers of the localities 


\section{Introduction}

The stratigraphy and nomenclature of the Phosphoria, Park City, and Shedhorn Formations in the western phosphate field have been described by McKelvey and others (1959). Analytical data obtained by the Geological Survey in the course of its study of the phosphate deposits in the Phosphoria Formation have been included in many publications. The chief sources of these data are included in the references at the end of this report.

The analytical data on the Phosphoria Formation that are presented here are those of the Geological Survey that so far have not been incorporated in published reports. They include complete rock analyses and analyses of many rare elements. The samples analyzed are identified by geographic location, by rock type, and by stratigraphic position in published stratigraphic sections. The purpose of the report is to make the data avallable for public utilization. 


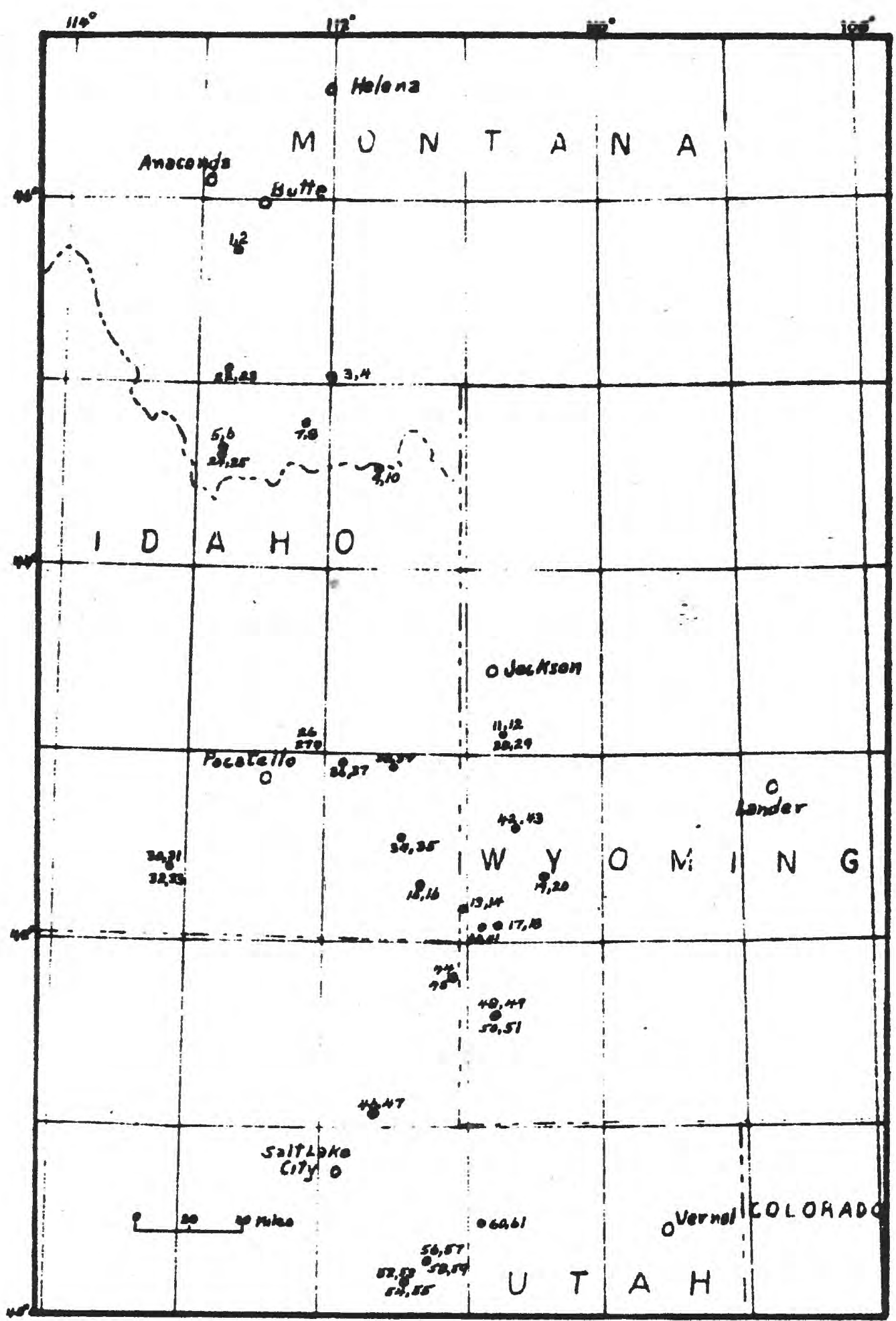

Figure 1.--Location of analyzed samples of quartz-s1licate rocks of table 1. Numbers are the analysis numbers. 


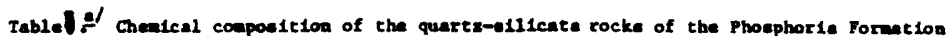

RTG 5580 RTC 5584 CWr 700 RSJ 717 WRL $57 \quad$ WRL 66

66

TSH 425

2

\begin{tabular}{|c|c|c|c|c|c|c|c|c|c|c|c|c|c|}
\hline $\mathrm{S1O}_{2}$ & 71.3 & 62.5 & 69.7 & 61.2 & 63.4 & 58.5 & 78.4 & 77.0 & 58.6 & 75.7 & 59.6 & 65.1 & 68.4 \\
\hline $\mathrm{Al}_{2} \mathrm{O}_{3}$ & 13.8 & 10.0 & 2.1 & 11.9 & 12.0 & 11.5 & 8.9 & 1.0 & 11.7 & 9.2 & 11.9 & 8.9 & 1.7 \\
\hline $\mathrm{Fe}_{20} \mathrm{O}_{3}$ sf & 1.6 & 4.0 & .6 & 5.4 & 4.9 & 5.4 & 3.9 & 3.1 & 4.4 & 4.3 & 4.4 & 3.9 & 3.5 \\
\hline $\mathbf{1}=0$ & .23 & .20 & 1.6 & 1.4 & 1.1 & 2.1 & .90 & 1.9 & 2.6 & 1.2 & .48 & .38 & 2.0 \\
\hline $\mathrm{H}_{80} \mathrm{O}$ & 1.6 & 1.5 & .26 & 1.7 & .83 & .93 & .53 & .06 & .79 & .72 & 1.3 & 1.1 & .42 \\
\hline $\mathrm{CaO}$ & 1.7 & 6.6 & 12.5 & 3.7 & 2.6 & 4.7 & .43 & 9.5 & 7.0 & 1.4 & 6.8 & 7.2 & 12.5 \\
\hline $\operatorname{Ma} 20$ & .11 & .14 & .22 & .16 & .51 & .66 & .14 & .24 & .24 & .18 & .24 & .20 & .14 \\
\hline $\mathrm{K}_{2} \mathrm{O}$ & 3.6 & 2.6 & .26 & 3.9 & 3.3 & 3.2 & 2.1 & .13 & 4.4 & 2.7 & 3.7 & 2.6 & .42 \\
\hline $\mathrm{H}_{2} \mathrm{O}$ tot & 3.7 & 5.5 & 4.4 & 4.8 & 6.2 & 7.5 & 3.9 & 1.0 & 3.6 & 4.1 & 5.2 & 3.7 & 1.0 \\
\hline $\mathrm{H}_{2} \mathrm{O}-$ & .5 & 1.8 & 0.1 & 0.8 & 1.2 & .9 & .4 & .05 & .7 & 0.6 & 1.2 & 0.8 & 0.1 \\
\hline $\mathrm{TLO}_{2}$ & .90 & .70 & .14 & .70 & .79 & .77 & .58 & .04 & .74 & .58 & .70 & .42 & .10 \\
\hline $\mathrm{I}_{2} \mathrm{O}_{5}$ & .68 & 3.7 & 6.6 & .48 & 1.7 & 3.0 & .37 & 7.2 & 4.9 & .95 & 4.3 & 3.8 & 4.8 \\
\hline $\mathrm{CO}_{2}$ & .65 & 1.23 & 3.22 & 2.81 & .26 & .29 & .04 & .35 & .33 & .23 & .42 & 1.44 & 5.21 \\
\hline $\mathrm{SO}_{3}$ & $\longrightarrow$ & - & - & - & - & - & .33 & - & - & .28 & - & - & - \\
\hline C1 & - & - & $\longrightarrow$ & - & - & - & - & - & - & - & - & - & - \\
\hline $\mathbf{F}$ & .28 & .50 & 0.75 & .13 & .23 & .38 &.$\infty$ & .74 & .60 & .11 & .58 & .42 & .50 \\
\hline $\begin{array}{l}\text { Ortanle sf } \\
\text { Matter }\end{array}$ & 2.0 & 2.6 & 1.2 & 4.0 & 5.4 & 6.3 & 2.8 & .5 & 4.3 & 2.4 & 3.2 & 2.1 & .5 \\
\hline $011 d$ & .10 & .13 & .28 & .20 & .50 & .84 & .25 & .02 & .10 & .20 & .15 & .10 & .20 \\
\hline v & .001 & .002 & .004 & .001 & .001 & .002 & .001 & .002 & .003 & .001 & .001 & .001 & .001 \\
\hline$\bullet 0$ & .001 & .001 & .004 & .004 & .003 & - & .002 & - & .005 & .001 & .002 & .002 & .001 \\
\hline 18 & .001 & .001 & .0001 & .0003 & .00003 & .00003 & .0002 & .0003 & .0003 & .00003 & .0001 & .0001 & .00003 \\
\hline as & .001 & .005 & .003 & .005 & .004 & .003 & & .002 & .002 & .003 & .006 & .003 & .006 \\
\hline B & .03 & .03 & $<.005$ & .03 & .0 .3 & .03 & .01 & $<.005$ & .03 & .01 & .01 & .03 & .01 \\
\hline he & .03 & .03 & .01 & פט. & .03 & .03 & .03 & .003 & .03 & .03 & .01 & .03 & .01 \\
\hline
\end{tabular}

Be

ca

.03

.01

.01
.003

.01

.01

.03

$\begin{array}{rrr} & <.0001 & .0001 \\ .001 & .0005 & .001\end{array}$

$\begin{array}{lll}.001 & .0005 & .001 \\ & .001 & .005\end{array}$

$\begin{array}{lll} & .001 & .005 \\ .03 & .01 & .01\end{array}$

$\begin{array}{lll}.03 & .01 & .01 \\ .01 & .02 & .01\end{array}$

$\begin{array}{lll}.003 & .02 & .03\end{array}$

$\begin{array}{lll}.0003 & .001 & .002\end{array}$

$\begin{array}{lll}.03 & .03 & .01\end{array}$

$\begin{array}{lll}.01 & .003 & .03\end{array}$ 
Tablelo/ Chesical composition of the quartz-silicste rocks of the Phosphoria Pormation--Continued

\begin{tabular}{|c|c|c|c|c|c|c|c|c|c|c|c|c|c|}
\hline & $\begin{array}{c}\text { VEM } 90 \\
14\end{array}$ & $\begin{array}{c}\text { RAH } 252 \\
15\end{array}$ & $\begin{array}{c}\text { RAS } 246 \\
16\end{array}$ & $\begin{array}{c}\text { LES } 27 \\
17\end{array}$ & $\begin{array}{c}\text { LES } 31 \\
18\end{array}$ & $\begin{array}{c}\text { RAS } 7204 \\
19\end{array}$ & $\begin{array}{c}\text { RAS } 7208 \\
20\end{array}$ & $\begin{array}{l}\text { Ave. } \\
\text { Retort } \\
21\end{array}$ & $\begin{array}{l}\text { WRL } 264 \\
22\end{array}$ & $\begin{array}{l}\text { WRL } 265 \\
23\end{array}$ & $\begin{array}{l}\text { WRL } 153 \\
24\end{array}$ & $\begin{array}{l}\text { WRL } 156 \\
25\end{array}$ & $\begin{array}{c}\text { HON } 2716 \\
26\end{array}$ \\
\hline $\mathrm{S1O}_{2}$ & 61.8 & 77.3 & 66.2 & 65.2 & 62.0 & 75.8 & 75.3 & 76.50 & 63.4 & 67.5 & 67.7 & 81.4 & 63.5 \\
\hline $\mathbf{A}_{2} \mathrm{O}_{3}$ & 9.7 & 4.6 & 9.4 & 2.5 & 5.8 & 7.6 & 6.6 & 8.04 & 10.1 & 9.8 & 3.0 & 3.9 & 11.2 \\
\hline $\mathrm{Fe}_{2} \mathrm{O}_{3} \stackrel{e}{=}$ & 4.8 & 4.3 & 3.8 & 3.2 & 2.7 & 2.9 & 2.9 & 3.70 & 5.8 & 1.9 & 3.5 & 3.6 & 3.7 \\
\hline Feo & .86 & 2.9 & .71 & 1.9 & .48 & .23 & .56 & 1.19 & 1.0 & .24 & 2.3 & 2.3 & .27 \\
\hline $\mathrm{MgO}$ & 1.1 & .86 & 1.9 & 1.8 & .81 & .92 & 1.0 & 1.01 & .57 & .60 & 2.6 & 1.0 & .35 \\
\hline $\mathrm{CaO}$ & 7.1 & 4.4 & 5.3 & 12.8 & 13,0 & 3.3 & 3.8 & 6.32 & 3.9 & 7.7 & 9.7 & 3.5 & 6.2 \\
\hline $\mathrm{Na}_{2} \mathrm{O}$ & .29 & .86 & .51 & .17 & .16 & .23 & .17 & .27 & .30 & .16 & .18 & .10 & 1.4 \\
\hline $\mathrm{R}_{2} \mathrm{O}$ & 2.9 & .80 & 2.4 & .57 & 1.6 & 2.3 & 2.0 & 2.27 & 1.8 & 1.2 & .70 & .94 & 2.7 \\
\hline $\mathrm{H}_{2} \mathrm{O}=\mathrm{LOt}$ & 4.4 & 1.5 & 4.3 & 1.1 & 2.1 & 1.5 & 3.2 & 3.64 & 5.9 & 3.8 & 1.5 & 1.7 & 3.8 \\
\hline $\mathrm{H}_{2} \mathrm{O}-$ & 1.0 & .2 & 1.0 & .05 & .2 & .7 & 0.8 & .66 & 2.3 & .7 & 0.4 & 0.2 & .4 \\
\hline $\mathrm{THO}_{2}$ & .54 & .31 & .54 & .12 & .32 & .36 & .33 & .48 & .73 & .68 & .20 & .26 & .72 \\
\hline $\mathrm{P}_{2} \mathrm{O}_{5}$ & 4.3 & 1.4 & 1.5 & 3.6 & 2.8 & 2.3 & 2.6 & 3.05 & 5.8 & 5.7 & 3.0 & 1.1 & 4.4 \\
\hline $\mathrm{CO}_{2}$ & .45 & 2.51 & 3.21 & 7.76 & 7.56 & .20 & 0.22 & 1.91 & .23 & .18 & 6.95 & 2.36 & .15 \\
\hline $\mathrm{SO}_{3}$ & - & .20 & - & - & .23 & - & - & .26 & - & .20 & .63 & .30 & .46 \\
\hline Cl & - & - & - & - & - & .02 & - & .02 & - & - & - & - & - \\
\hline$P$ & .58 & .17 & .33 & .43 & .38 & .30 & .37 & .39 & .43 & .58 & .32 & .11 & .45 \\
\hline $\begin{array}{l}\text { Organic sf } \\
\text { Mateer }\end{array}$ & 3.0 & 1.1 & 3.0 & 1.0 & 1.5 & 2.0 & 2.6 & 2.57 & 2.4 & 1.7 & 1.1 & 1.6 & 2.2 \\
\hline oul $\stackrel{d}{ }$ & .20 & .10 & .22 & .10 & .10 & .20 & .05 & .197 & .12 & .20 & .32 & .04 & .15 \\
\hline $\mathbf{v}$ & .001 & .001 & .001 & .001 & .001 & .001 & .001 & .0014 & .002 & .002 & .002 & .001 & .001 \\
\hline ev & - & .001 & .002 & .001 & - & .002 & .003 & .001 & .004 & .003 & - & - & .002 \\
\hline As & .00001 & $<.00001$ & .00001 & $<.00001$ & $<.00001$ & .00003 & .0001 & $\begin{array}{l}.00003- \\
.0001\end{array}$ & .001 & .0003 & .0003 & .00003 & .0003 \\
\hline As & .003 & .004 & .001 & .006 & .004 & .006 & .006 & .0038 & .008 & .008 & .023 & .001 & .002 \\
\hline B & .03 & .01 & .03 & .01 & .01 & .01 & .01 & $.03-.01$ & .01 & .01 & .01 & .01 & .01 \\
\hline Ba & .01 & .03 & .03 & .01 & .03 & .03 & .01 & .03 & .01 & .01 & .01 & .03 & .03 \\
\hline Be & $<.00005$ & $<.00005$ & $<.00005$ & $<.00005$ & $<.00005$ & $<.00005$ & $<.00005$ & $<.00005$ & $<.00005$ & $<.00005$ & $<.00005$ & $<.00005$ & .400005 \\
\hline cd & $<.005$ & $<.005$ & $<.005$ & $<.005$ & $<.005$ & $<.005$ & $<.005$ & $<.005$ & $<.005$ & $<.005$ & $<.005$ & $<.005$ & $<.005$ \\
\hline co & $<.001$ & $<.001$ & $<.001$ & $<.001$ & $<.001$ & $<.001$ & $<.001$ & $<.001$ & $<.001$ & $<.001$ & $<.001$ & $<.001$ & $<.001$ \\
\hline $\mathrm{Cr}$ & .1 & .01 & .1 & .03 & .03 & .1 & .1 & .1 & .1 & .03 & .01 & .01 & .1 \\
\hline $\mathrm{Cu}$ & .01 & .01 & .003 & .01 & .003 & .01 & .003 & .003 & .03 & .01 & .01 & .01 & .003 \\
\hline $\mathrm{Ga}$ & .001 & $<.001$ & .001 & $<.002$ & $<.001$ & .001 & $<.001$ & .001 & .001 & .001 & $<.001$ & $<.001$ & .001 \\
\hline La & .01 & $<.003$ & .003 & $<.003$ & $<.003$ & .003 & $<.003$ & $<.003-.01$ & .003 & .003 & $<.003$ & $<.003$ & .01 \\
\hline 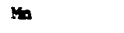 & .03 & .03 & .01 & .03 & .03 & .01 & .003 & $.03-.01$ & .003 & .001 & .03 & .01 & .001 \\
\hline to & .001 & $<.0005$ & $<.0005$ & $<.0005$ & $<.0005$ & .003 & .01 & $.003-.01$ & .003 & $<.0005$ & .003 & .003 & .01 \\
\hline N1 & .01 & .003 & .01 & .003 & .003 & .03 & .003 & .003 & .001 & .0003 & .003 & .01 & .01 \\
\hline $\mathbf{P b}$ & .03 & $<.001$ & $<.001$ & .003 & $<.01$ & .001 & .003 & $<.001-.001$ & 1.01 & .01 & .001 & $<.001$ & .001 \\
\hline $\mathbf{s b}$ & .0005 & .0002 & $<0001$ & $<.0001$ & $<.0001$ & $<.0001$ & $<.0001$ & .0002 & .001 & .0003 & .0002 & .0003 & .0005 \\
\hline Sc & .001 & $<.0005$ & .001 & $<.0005$ & .001 & .001 & .001 & .001 & .001 & .001 & .001 & .001 & .001 \\
\hline se & .002 & .001 & .0003 & .001 & .0003 & .0005 & .001 & .001 & .0003 & .0001 & .003 & .001 & .003 \\
\hline Sr & .01 & .01 & .01 & .01 & .01 & .03 & .01 & .01 & .01 & .01 & .01 & .01 & .03 \\
\hline v & .003 & .001 & .01 & .001 & .001 & .01 & .01 & .01 & .01 & .01 & .003 & .001 & .01 \\
\hline$x$ & .01 & .003 & .01 & .003 & .003 & .01 & .003 & .01 & .003 & .003 & .003 & .003 & .01 \\
\hline Yb & .001 & .0003 & .0003 & .0001 & .0003 & .001 & .0003 & .0003 & .0003 & .0003 & .0003 & .0003 & .001 \\
\hline $2 n$ & .1 & $<.008$ & $<.008$ & .01 & .01 & .01 & .03 & .01 & $<.008$ & $<.008$ & $<.008$ & .01 & .03 \\
\hline $2 r$ & .01 & .01 & .01 & .001 & .003 & .01 & .01 & .01 & .01 & .03 & .01 & .01 & .03 \\
\hline
\end{tabular}


RAS 6336 RAS 6455 MAN 6487 MAH 6425 RAS 6426 RAS 6432 RGN 6452 WOM 2974 WOM 2981 HWP 4340 RSJ 4331 MET 4300 RAS 4273

$\begin{array}{llllllllllll}27 & 28 & 29 & 30 & 31 & 32 & 33 & 34 & 35 & 36 & 37 & 38\end{array}$

\begin{tabular}{|c|c|c|c|c|c|c|c|c|c|c|c|c|c|}
\hline $\mathrm{SiO}_{2}$ & 62.7 & 61.1 & 63.1 & 72.4 & 65.6 & 58.6 & 67.0 & 72.3 & .71 .8 & 63.5 & 70.5 & 56.6 & 75.0 \\
\hline $\mathrm{Al}_{2} \mathrm{O}_{3}$ & 10.5 & 7.5 & 9.5 & 13.1 & 8.0 & 7.9 & 9.9 & 11.7 & 11.2 & 11.2 & 12.5 & 10.6 & 9.4 \\
\hline $\mathrm{Fe}_{2} \mathrm{O}_{3}$ ef & 2.6 & 2.6 & 4.1 & 2.7 & 3.3 & 2.5 & 3.8 & 4.0 & 4.0 & 3.9 & 5.0 & 3.9 & 2.9 \\
\hline Peo & .21 & .89 & 1.4 & .21 & .39 & .08 & .47 & .33 & .61 & .31 & .48 & .32 & .35 \\
\hline $\mathrm{MgO}$ & .83 & 1.7 & .59 & .88 & .61 & .78 & .84 & .60 & .40 & .52 & .56 & .69 & .64 \\
\hline $\mathrm{CaO}$ & 7.7 & 10.1 & .21 & .76 & 8.8 & 12.9 & 4.3 & .54 & 1.7 & 5.5 & .49 & 8.0 & 2.6 \\
\hline $\mathrm{Na}_{2} \mathrm{O}$ & .16 & .25 & .23 & .21 & .30 & 1.8 & .50 & 1.0 & 1.5 & 1.1 & 1.0 & 1.2 & 1.5 \\
\hline $\mathrm{K}_{2} \mathrm{O}$ & 2.3 & 2.5 & 3.0 & 3.6 & 2.1 & 1.3 & 2.1 & 3.0 & 2.7 & 2.8 & 3.2 & 2.6 & 1.7 \\
\hline $\mathrm{H}_{2} \mathrm{O}$ tot & 4.5 & 2.6 & 5.6 & 5.1 & $4.1^{\circ}$ & 3.1 & 5.7 & 4.8 & 3.8 & 4.1 & 2.2 & 1.7 & 3.7 \\
\hline $\mathrm{H}_{2} \mathrm{O}^{-}$ & 0.8 & 0.5 & 1.7 & .8 & .8 & .7 & 1.4 & 1.5 & .7 & 1.4 & 1.0 & 1.3 & .4 \\
\hline $\mathrm{THO}_{2}$ & .62 & .54 & 1.0 & .74 & .39 & .48 & .62 & .78 & .76 & .74 & .81 & .82 & .60 \\
\hline $\mathrm{P}_{2} \mathrm{O}_{5}$ & 4.9 & 8.4 & .76 & .64 & 6.8 & 9.6 & 3.5 & .19 & 1.3 & 3.7 & .27 & 4.7 & 1.7 \\
\hline $\mathrm{CO}_{2}$ & 0.61 & 0.53 & .03 & .05 & .19 & .39 & .28 & .02 & .07 & .18 & .16 & .34 & .13 \\
\hline $\mathrm{SO}_{3}$ & - & - & - & - & - & - & - & .21 & .32 & .46 & - & - & - \\
\hline CI & - & - & - & - & - & - & - & - & - & .04 & - & - & - \\
\hline $\mathbf{F}$ & .50 & 1.15 & .11 & .20 & .68 & .93 & .33 & .13 & .15 & .45 & .07 & .50 & .28 \\
\hline $\begin{array}{l}\text { orgenic } s / \\
\text { metcer }\end{array}$ & 1.0 & 1.8 & 11.6 & 2.1 & 1.5 & 1.1 & .8 & 1.4 & 1.7 & 2.0 & 1.7 & 5.0 & 1.9 \\
\hline $011 \stackrel{d \prime}{\prime}$ & .10 & .15 & .22 & .10 & .13 & .20 & .25 & .02 & .38 & .20 & .06 & .18 & .15 \\
\hline $\mathbf{u}$ & .003 & .005 & .003 & .001 & .002 & .003 & .002 & .001 & .001 & .001 & .001 & .002 & .001 \\
\hline eu & .001 & .00 .5 & - & .003 & .002 & .005 & - & .002 & .002 & .001 & .001 & .004 & - \\
\hline As & .001 & .001 & .001 & .001 & .001 & .0003 & .0003 & .0001 & .0001 & .0001 & .00001 & .001 & .00003 \\
\hline As & .003 & .02 & .005 & .003 & .002 & .002 & .002 & .003 & .002 & .003 & .002 & .005 & .004 \\
\hline B & .01 & $\$ .005$ & .01 & .03 & .01 & .003 & .01 & .01 & .01 & .01 & .01 & .01 & .03 \\
\hline Be & .03 & .01 & .01 & .03 & .03 & .01 & .3 & .03 & .03 & .03 & .03 & .03 & .03 \\
\hline Be & $<.00005$ & $<.00005$ & $<.00005$ & $<.00005$ & $<.00005$ & $<.00005$ & $<.00005$ & $<.00005$ & $\therefore<.00005$ & $<.00005$ & $<.00005$ & .0001 & $<.00005$ \\
\hline$c d$ & $<.005$ & .01 & $<.005$ & $<.005$ & $<.005$ & $<.005$ & $<.005$ & $<.005$ & $<.005$ & $<.005$ & $<.005$ & $<.005$ & $<.005$ \\
\hline Co & $<.001$ & $<.001$ & $<.001$ & $<.001$ & $<.001$ & .001 & $<.001$ & $<.001$ & $<.001$ & $<.001$ & $<.001$ & $<.001$ & .001 \\
\hline $\mathrm{Cr}$ & .3 & .03 & .1 & .3 & .1 & .03 & .1 & .03 & .1 & .1 & .03 & .3 & .03 \\
\hline $\mathrm{Cu}$ & .01 & .01 & .003 & .01 & .01 & .01 & .01 & .001 & .003 & .003 & .003 & .01 & .003 \\
\hline $\mathrm{Ga}$ & .001 & .001 & .001 & .001 & .001 & $<.001$ & .001 & $<.001$ & .001 & .001 & .001 & .001 & .001 \\
\hline La & .003 & .01 & .003 & .01 & $<.003$ & .003 & .003 & $<.003$ & .003 & .003 & $<.003$ & .003 & .003 \\
\hline Mn & .03 & .003 & .001 & .003 & .001 & .03 & .001 & .003 & .01 & .03 & .01 & .003 & .03 \\
\hline$M$ & .003 & .03 & .1 & .003 & $<.0005$ & .003 & .003 & .003 & .003 & .003 & .003 & .01 & .01 \\
\hline M1 & .01 & .03 & .003 & .03 & .01 & .1 & .03 & .01 & .01 & .03 & .01 & .01 & .03 \\
\hline $\mathrm{Pb}$ & .001 & $<.001$ & .001 & .001 & .001 & $<.001$ & $<.001$ & .001 & .001 & $<.001$ & $<.001$ & .001 & $<.001$ \\
\hline Sb & .001 & .001 & .001 & .0003 & .0001 & .0005 & .0002 & .0003 & .0003 & .0001 & .0005 & .0005 & .0001 \\
\hline Sc & .001 & .001 & .001 & .003 & .001 & .001 & .001 & .001 & .001 & .001 & .001 & .001 & .001 \\
\hline Se & .001 & .003 & .002 & .0005 & .0005 & .0003 & .0003 & .01 & .0005 & .0007 & .0005 & .005 & .0007 \\
\hline $\mathbf{S x}$ & .01 & .03 & .01 & .03 & .01 & .01 & .03 & .01 & .01 & .01 & .01 & .03 & .01 \\
\hline$v$ & .01 & .01 & .1 & .01 & .01 & .01 & .01 & .003 & .003 & .01 & .003 & .01 & .01 \\
\hline$y$ & .01 & .03 & .003 & .01 & .003 & .01 & .003 & .003 & .003 & .01 & .003 & .01 & .01 \\
\hline$Y_{b}$ & .001 & .001 & .0003 & .001 & .0003 & .0003 & .0003 & .0003 & .0003 & .001 & .0003 & .001 & .001 \\
\hline $2 n$ & .03 & .1 & $<.008$ & .01 & .03 & .03 & .03 & .03 & .03 & .01 & $<.008$ & .03 & .01 \\
\hline $2 r$ & .03 & .03 & .03 & .03 & .003 & .01 & .01 & .03 & .03 & .03 & .03 & .01 & .03 \\
\hline
\end{tabular}




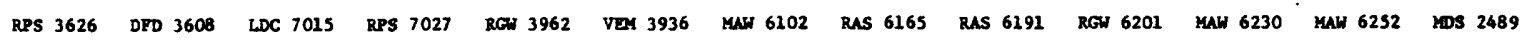

$\begin{array}{llllllllllllll}40 & 41 & 42 & 43 & 44 & 45 & 46 & 47 & 48 & 49 & 50 & 51 & 52\end{array}$

\begin{tabular}{|c|c|c|c|c|c|c|c|c|c|c|c|c|c|}
\hline $\mathrm{SIO}_{2}$ & 59.0 & 63.4 & 42.6 & 66.7 & 64.4 & 68.6 & 66.5 & 66.3 & $\$ 1.3$ & 52.4 & 60.2 & 74.4 & 70.2 \\
\hline $\mathrm{Al}_{2} \mathrm{O}_{3}$ & 13.2 & 9.2 & 6.9 & 11.8 & 3.6 & 13.3 & 10.1 & 6.5 & 11.8 & 12.0 & 12.3 & 8.2 & 5.0 \\
\hline 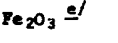 & 4.6 & 3.7 & 2.0 & 3.4 & 1.2 & 4.2 & 3.6 & 2.8 & 2.8 & 1.2 & 4.2 & 2.4 & 2.9 \\
\hline Peo & 1.8 & 1.0 & .24 & 1.4 & .40 & 1.0 & .75 & .84 & .37 & .45 & 1.2 & .32 & 2.6 \\
\hline $\mathrm{MgO}$ & 1.1 & 3.3 & 8.4 & .84 & 2.4 & 1.2 & 2.4 & .76 & 1.7 & 1.1 & 1.1 & .80 & 1.9 \\
\hline $\mathrm{CaO}$ & 3.3 & 6.4 & 13.1 & 1.5 & 12.1 & .62 & 4.8 & 9.6 & 12.2 & 12.4 & 1.1 & 4.6 & 8.2 \\
\hline $\operatorname{Na} 20$ & .54 & 1.2 & .24 & .23 & .21 & .22 & 2.6 & .29 & .58 & .66 & .72 & .36 & .22 \\
\hline $\mathrm{x}_{2} \mathrm{O}$ & 3.6 & 2.2 & 1.8 & 3.1 & 1.0 & 3.9 & 2.2 & 1.7 & 3.5 & 3.7 & 2.9 & 1.6 & 1.4 \\
\hline $\mathrm{H}_{2} \mathrm{O}$ & 6.7 & 2.4 & 2.9 & 5.8 & 1.4 & 5.3 & 2.0 & 3.1 & 3.8 & 4.6 & 7.2 & 3.9 & 2.7 \\
\hline $\mathrm{H}_{2} \mathrm{O}^{-}$ & 1.8 & .3 & .6 & 1.7 & .2 & 1.2 & .1 & 1.0 & 0.8 & 1.0 & 2.0 & 1.0 & .5 \\
\hline $\mathrm{THO}_{2}$ & 1.0 & .60 & .47 & 1.0 & .20 & .90 & .64 &. .38 & .71 & .66 & 1.1 & .37 & .22 \\
\hline $\mathrm{P}_{2} \mathrm{O}_{5}$ & .39 & .49 & .77 & .63 & 4.3 & .19 & .72 & 5.8 & 7.4 & 8.4 & 1.1 & 2.4 & 1.7 \\
\hline $\mathrm{CO}_{2}$ & 1.54 & 7.32 & .83 & .29 & 6.92 & .08 & 4.92 & 1.39 & 2.26 & 0.83 & .13 & .98 & 6.18 \\
\hline $\mathrm{SO}_{3}$ & 1.02 & .15 & - & - & .38 & - & - & - & - & - & - & - & - \\
\hline Cl & - & - & - & - & - & - & - & - & .07 & - & - & .15 & - \\
\hline $\mathbf{F}$ & .23 & .18 & .13 & .20 & .40 & .18 & .10 & .56 & 0.88 & 0.82 & .08 & .27 & .18 \\
\hline $\begin{array}{l}\text { Organse } \frac{c}{} \\
\text { Matter }\end{array}$ & 5.8 & 1.5 & 2.6 & 3.9 & 1.3 & 9.1 & 1.1 & 1.6 & 1.4 & 1.2 & 8.4 & 1.8 & 2.1 \\
\hline $011 d$ & .15 & .10 & .10 & .15 & .05 & .10 & .08 & .25 & .20 & .12 & .44 & .14 & .11 \\
\hline $\mathbf{u}$ & .002 & .001 & .001 & .002 & .001 & .001 & .001 & .001 & .001 & .003 & .002 & .001 & .001 \\
\hline ell & .003 & .002 & - & .005 & .001 & - & .001 & .002 & .003 & - & - & .001 & - \\
\hline 18 & .002 & .0003 & .0003 & .001 & .001 & .001 & .0001 & .0003 & .0001 & .001 & .001 & .001 & .00001 \\
\hline As & .02 & .005 & .003 & .002 & .001 & .003 & .002 & .002 & $<.002$ & .001 & .01 & .002 & .001 \\
\hline 8 . & .03 & .03 & $<.005$ & .01 & .01 & .03 & .01 & .01 & .03 & .03 & .03 & .01 & .01 \\
\hline Ba & .03 & .01 & .003 & .03 & .01 & .03 & $.0 s$ & .01 & .us & .01 & .03 & .01 & .01 \\
\hline Be & .0001 & $<.00005$ & $<.00005$ & .0001 & $<.00005$ & .0001 & $<.0000 s$ & $<.00005$ & .0001 & .0001 & $<.00005$ & $<.00005$ & $<.0000 s$ \\
\hline cd & $<.005$ & $<.005$ & $<.005$ & $<.005$ & $<.005$ & $<.005$ & $<-005$ & $<.005$ & $<.005$ & $<.003$ & $<.005$ & $<.005$ & $<.005$ \\
\hline co & .003 & $<.001$ & $<.001$ & $<.001$ & $<.001$ & $<.001$ & .001 & $<.001$ & $<.001$ & $<.001$ & $<.001$ & $<.001$ & $<.001$ \\
\hline Cr & .1 & .03 & .03 & .1 & .03 & .1 & .03 & .1 & .1 & .1 & .1 & .1 & .03 \\
\hline Cu & .01 & .003 & .003 & .003 & .003 & .003 & .003 & .003 & .003 & .003 & .01 & .003 & .003 \\
\hline Ga & .001 & $<.001$ & $<.001$ & .001 & $<.001$ & .001 & .001 & $<.001$ & .001 & .001 & .001 & .001 & $<.001$ \\
\hline La & $<.003$ & $<.003$ & $<.003$ & .003 & $-<.003$ & .003 & $<.003$ & $<.003$ & .003 & .003 & .003 & $<.003$ & $<.003$ \\
\hline Mn & .01 & .03 & .003 & .001 & .003 & .001 & .02 & .003 & .02 & .001 & .003 & .01 & .01 \\
\hline Ho & .03 & .003 & .003 & .03 & $<.0005$ & .03 & .003 & $<.0005$ & $<.0005$ & $<.0005$ & .03 & $<.0005$ & $<.0005$ \\
\hline $\mathbf{M 1}$ & .03 & .01 & .003 & .01 & .01 & .01 & .02 & .001 & .01 & .01 & .01 & .01 & .003 \\
\hline $\mathbf{P b}$ & .003 & $<.001$ & $<.001$ & .001 & $<.001$ & .003 & $<.002$ & $<.001$ & .001 & $<.001$ & .001 & $<.001$ & $<.001$ \\
\hline $\mathbf{s b}$ & .0003 & .0005 & .0013 & .0015 & .0002 & .0007 & .0001 & .0001 & .0002 & .0002 & .006 & .0001 & $<.0001$ \\
\hline Sc & .003 & .001 & .002 & .003 & $<.0005$ & .003 & .001 & .001 & .001 & .01 & .003 & .001 & $<.000 s$ \\
\hline se & .007 & .001 & .002 & .0007 & .0003 & .003 & .0007 & .0001 & .0005 & .001 & .003 & .0007 & .0005 \\
\hline Sr & .01 & .01 & .003 & .01 & .01 & .01 & .01 & .01 & .01 & .03 & .01 & .01 & .01 \\
\hline $\mathbf{v}$ & .1 & .003 & .01 & .1 & .003 & .1 & .003 & .003 & .01 & .01 & .1 & .003 & .001 \\
\hline$Y$ & .01 & .003 & .003 & .003 & .003 & .003 & .003 & .003 & .01 & .01 & .01 & .003 & .003 \\
\hline $\mathbf{Y b}$ & .001 & .0003 & .0003 & .0003 & .0001 & .0003 & .0003 & .0003 & .0003 & .001 & .001 & .0003 & .0003 \\
\hline $2 n$ & .03 & .01 & .03 & .03 & .03 & .03 & $<.008$ & .03 & .01 & .03 & $<.008$ & .03 & $<.008$ \\
\hline $2 x$ & .03 & .01 & .01 & .03 & .003 & .03 & .03 & .01 & .01 & .01 & .03 & .003 & .002 \\
\hline
\end{tabular}




\begin{tabular}{|c|c|c|c|c|c|c|c|c|c|c|c|}
\hline & $\begin{array}{c}\text { DPS } 2455 \\
53\end{array}$ & $\begin{array}{c}\text { GrA } 2340 \\
54\end{array}$ & $\begin{array}{c}\text { GFA } 2570 \\
55\end{array}$ & $\begin{array}{c}\text { RSS } 2224 \\
56\end{array}$ & $\begin{array}{c}\text { DPS } 2381 \\
57\end{array}$ & $\begin{array}{c}\text { IDS } 2181 \\
58\end{array}$ & $\begin{array}{c}\text { LES } 2290 \\
59\end{array}$ & $\begin{array}{c}\text { Jง } 2015 \\
60\end{array}$ & $\begin{array}{c}J B C 2163 \\
62\end{array}$ & $\begin{array}{l}\text { Ave. Meade } b / \\
\text { Peak } \\
62\end{array}$ & $\begin{array}{c}\text { Axes b bor is } \\
63\end{array}$ \\
\hline $\mathrm{S1O}_{2}$ & 64.1 & 73.2 & 65.3 & 61.8 & 71.8 & 74.1 & 61.8 & 63.3 & 69.2 & 63.36 & 66.12 \\
\hline $\mathrm{AI}_{2} \mathrm{O}_{3}$ & 4.2 & 3.2 & 4.5 & 4.1 & 2.9 & 5.4 & 5.1 & 4.7 & 3.7 & 8.32 & 8.22 \\
\hline $\mathrm{Fe}_{2} \mathrm{O}_{3}$ el & 2.4 & 2.6 & 2.9 & 3.2 & 2.5 & 2.3 & 2.8 & 2.6 & 2.9 & 3.18 & 3.35 \\
\hline Feo & 1.0 & 1.2 & 1.8 & 1.6 & 1.6 & .72 & 1.4 & 1.3 & 1.5 & .89 & .99 \\
\hline $\mathrm{MgO}$ & 3.3 & .32 & .81 & 3.0 & .30 & .60 & 3.6 & 4.4 & 2.3 & 1.51 & 1.34 \\
\hline $\mathrm{CaO}$ & 10.6 & 10.3 & 12.3 & 11.8 & 11.0 & 6.7 & 10.5 & 8.7 & 9.7 & 6.9 & 6.71 \\
\hline $\mathrm{N}={ }_{2} \mathrm{O}$ & .19 & .22 & .34 & .31 & .20 & .32 & .22 & .18 & .23 & .60 & 4.77 \\
\hline $\mathrm{x}_{2} \mathrm{O}$ & 1.1 & .52 & 1.1 & 1.0 & .56 & 1.2 & 1.3 & 1.2 & .76 & 2.04 & 2.12 \\
\hline $\mathrm{H}_{2} \mathrm{O}$ tot & 2.1 & 1.8 & 2.9 & 2.0 & 1.6 & 2.6 & 2.2 & 2.6 & 1.3 & 3.46 & 3.52 \\
\hline $\mathrm{H}_{2} \mathrm{O}-$ & 0.3 & 0.3 & .4 & .4 & .1 & 0.8 & 0.6 & 0.3 & .05 & .81 & .76 \\
\hline $\mathrm{IHO}_{2}$ & .20 & .12 & .20 & .20 & .13 & .26 & .27 & .20 & .18 & .54 & .52 \\
\hline $\mathrm{P}_{2} \mathrm{O}_{5}$ & 1.8 & 5.0 & 6.7 & 2.3 & 2.3 & 4.3 & 1.7 & 1.6 & 2.7 & 3.23 & 3.17 \\
\hline $\mathrm{CO}_{2}$ & 9.43 & 2.84 & 3.04 & 9.70 & 6.35 & .92 & 9.35 & 9.04 & 6.86 & 2.60 & 2.37 \\
\hline $\mathrm{SO}_{3}$ & - & .41 & .64 & - & - & - & - & .49 & - & .44 & .39 \\
\hline C1 & - & - & .02 & - & - & - & .02 & - & - & .06 & .05 \\
\hline F & .23 & .55 & .68 & .23 & .23 & .37 & .20 & 0.20 & .30 & .36 & .37 \\
\hline $\begin{array}{l}\text { Organie } c / \\
\text { Kacter }\end{array}$ & 2.0 & .5 & 1.8 & 2.1 & 1.4 & 1.4 & 3.1 & 3.1 & 1.5 & 2.38 & 2.44 \\
\hline $011 \subseteq$ & .12 & .10 & .02 & .05 & .06 & .20 & .30 & .10 & .20 & .13 & .17 \\
\hline $\mathbf{v}$ & .001 & .001 & .001 & .001 & .001 & .001 & .001 & .001 & .001 & .0015 & .0015 \\
\hline at & - & - & - & - & - & - & - & .002 & - & .002 & .001 \\
\hline
\end{tabular}

\begin{tabular}{|c|c|c|c|c|c|c|c|c|c|c|c|}
\hline$A_{B}$ & .00003 & .00003 & .00003 & .00003 & $\cdot .00003$ & .0001 & $<.00001$ & .0003 & $<.00001$ & .002 & .001 \\
\hline As & $<.001$ & .002 & .001 & $<.001$ & $<.001$ & .002 & .002 & .002 & .002 & .006 & .004 \\
\hline B & .01 & .01 & .01 & .01 & .01 & .01 & .01 & .01 & .01 & .01 & .01 \\
\hline Bะ & .01 & .01 & .01 & .01 & .01 & .003 & .01 & .01 & .01 & .01 & .03 \\
\hline Be & $<.00003$ & $<.00005$ & $<.00005$ & $<.0000 s$ & $<.00005$ & $<.00005$ & $<.00005$ & $<.00005$ & $<.00005$ & $<.00005$ & $<.00005$ \\
\hline cd & $<.005$ & $<.005$ & $<.005$ & $<.005$ & $<.005$ & $<.005$ & $<.005$ & $<.005$ & $<.005$ & $<.005$ & $<.005$ \\
\hline$\omega_{0}$ & $<.001$ & $<.001$ & $<.001$ & $<.001$ & $<.001$ & $<.001$ & $<.001$ & $<.001$ & $<.001$ & $<.001$ & $<.001$ \\
\hline Cr & .03 & .03 & .1 & .03 & .03 & .1 & .03 & .1 & 03 & .1 & .1 \\
\hline $\mathrm{Cu}$ & .003 & .003 & .01 & .003 & .003 & .003 & .003 & .003 & .003 & .003 & .003 \\
\hline Ge & $<.001$ & $<.001$ & $<.001$ & $<.001$ & $<.001$ & $<.001$ & $<.001$ & $<.001$ & $<.001$ & .001 & .001 \\
\hline La & $<.003$ & $<.003$ & $<.003$ & $<.003$ & $<.003$ & $<.003$ & $<.003$ & $<.003$ & $<.003$ & $<.003$ & $<.003$ \\
\hline Mn & .01 & .01 & .01 & .01 & .01 & .001 & .01 & .01 & .01 & .01 & .01 \\
\hline 10 & $<.0005$ & $<.0005$ & $<.0005$ & $<.000 s$ & .003 & $<.000 s$ & $<.0005$ & $<.0005$ & $<.0005$ & $<.0005$ & .003 \\
\hline ME & .003 & .003 & .003 & .003 & .003 & .001 & .003 & .003 & .003 & .01 & .01 \\
\hline $\mathrm{Pb}$ & $<.001$ & $<.001$ & $<.001$ & $<.001$ & $<.001$ & $<.001$ & $<.001$ & $<.001$ & $<.001$ & $<.001$ & $<.001$ \\
\hline $\mathbf{s b}$ & .0001 & .0001 & .0001 & .0003 & .0005 & $<.0002$ & .0001 & $<.0001$ & .0001 & .0004 & .0004 \\
\hline Sc & $<.0005$ & $<.0005$ & .001 & .001 & .001 & .001 & $<.0005$ & .001 & $<.0005$ & .001 & .001 \\
\hline Se & .0003 & .0003 & .002 & .0003 & .0003 & .0001 & .0001 & .0001 & .0003 & .0014 & .0013 \\
\hline SE & .01 & .01 & .01 & .01 & .01 & .01 & .01 & .01 & .01 & .01 & .01 \\
\hline$v$ & .001 & .001 & .001 & .001 & $<.001$ & .003 & .001 & .001 & $<.001$ & .01 & .01 \\
\hline $\mathbf{Y}$ & .001 & .003 & .003 & .001 & .001 & .003 & .001 & .001 & .001 & .003 & .003 \\
\hline$y b_{b}$ & .0001 & .0003 & .0003 & .0001 & .0001 & .0003 & .0001 & .0001 & .0001 & .0003 & .0003 \\
\hline $2 n$ & $<.008$ & $<.008$ & $<.008$ & $<.008$ & $<.008$ & $<.008$ & $<.008$ & .01 & $<.008$ & .03 & .03 \\
\hline 25 & .001 & .001 & .003 & .001 & .001 & .003 & .003 & .001 & .003 & .03 & .01 \\
\hline
\end{tabular}


af Chesical determinatione of $\mathrm{SiO}_{2}, \mathrm{Al}_{2} \mathrm{O}_{3}, \mathrm{Fe}_{2} \mathrm{O}_{3}, \mathrm{FeO}, \mathrm{MgO}, \mathrm{CaO}, \mathrm{Na} \mathrm{O}_{2}, \mathrm{~K}_{2} \mathrm{O}, \mathrm{THO} \mathrm{O}_{2}$, and $\mathrm{P}_{2} \mathrm{O}_{5}$ by P.L.D. Elmore, S. D. Botts, P. W. Scott, K. E. White and S. Bethea; of U.F, $\mathrm{CO}_{2}, \mathrm{H}_{2} \mathrm{O}^{\text {(total) }}, \mathrm{H}_{2} \mathrm{O}^{-}$, Organic Matter, and 011 by G. Edg1ngton, A. Schrenk, W. Tucker, R. Moore and G. Daniels; and of $\mathrm{SO}_{3}$ and $\mathrm{Cl}$ by S. M. Berthold.

Radiometric determinatione of U (eU) by B. A. McCall.

Spectrographic deceraination of elements listed alphabetically (excluding As, Sb, and Se) by Charles Annell.

Elements looked for but not detected (sensitivity limit shown in parentheses): Au (.001), B1 (.005), Ce (.03), Cs(.8), Dy (.006), Er (.003), Eu (.003), Gd (.006), Ge (.001), He (.007), Hg (.08), Ho (.001), In (.0004), Ir (.03), L1 (.01), Le (.005), Nb (.001), Nd (.006), Os (.1), $\operatorname{Pd}(.003)$, Pr $(.01)$, Pe $(.003)$, Rb (T.), Re (.04), Rh $(.004)$, Ru $(.008)$, Sal $(.008)$, Ta $(.1)$, Tb $(.01)$, Te $(.08)$, Th $(.05)$, TI $(.04)$, Tm $(.001)$ and $w(.05)$.

Geochemical determinatione of As, Sb and Se by H. E. Crowe, J.z. Swick, and R. R. Beins.

b/ Model values used for alnor elements Ilsted alphabetically, except for average values of As, Sb and Se.

c/ Organic matter by partial conbustion

d' O1l by distillation

ef Tocal Iron as $\mathrm{Fe}_{2} \mathrm{O}_{3}$

1. Sample No. RFG 5580, Lab No. 81410, Retort Member, LaMarch Gulch, Beaverhead County, Montane (Creasaan and Swanson, 1964, P. 553.)

2. Sample No. RFG 5584, Lab No. D-54752, Retort Member, LaMarch Gulch, Beaverhead County, Montana (Cressman and Swanson, 1964, p. 553.)

2. Sample No. RFG 588, Lab No. D-5452, Retort Member, LaMarch Gulch, Beaverhead County, Montana (Cressman and Swanson, 1964 , P.

3. Sample No. CWT 700, Lab No. D-42500, Retort Member, Canyon Camp, Madison County, Montana (Cresgan and Swanson, 1964, p. 511.)
4. Sample No. RSJ 717, Lab No. D-42483, Retort Member, Canyon Camp, Madison County, Montana (Cressasn and Swanson, 1964, p. 510.)

4. Sample No. RSJ 717, Lab No. D-42483, Retort Meaber, Canyon Camp, Madison County, Montana (Cressaan and Swanson, 1964, P, 510.)
5. Sample No. WRL 57, Lab. No. 9612, Retort Meaber, Hidden Pasture Creek, Beaverhead County, Montana (Swanson and others, 1953, p. 27.)

6. Sample WRL 66, Lab. No. 9621, Recort Mener, Hidden Pasture Creek. Beaverheed County, Montana (Swanson and others, 1953, p. 27.)

7. Sample No. FSH 425, Lab No. 6750, Retort Meaber, Sawtooth Mountain, Beaverhead County, Hontana (Cresanan and Swaneon, ig64, p.445.)

8. Sample No. FSH 438, Lab No. 6763, Retort Meaber. Sawtooth Mountain, Beaverhead County, Montans (Cressuan and Swanson, 1964, p.445.)

9. Sample No. OAP 520, Lab. Ho. 19330. Retort Merber, Centennial No. 2, Clerk County, Idaho (Cressman and Swanson, 1964 , p. 469.)

10. Sample No. OAP 525, Lab. No. 19335, Retort Member, Cencennial to. 2, Clark County, Idaho (Cresaman and Swaneon, 1964,
11 . Sample No. RAS 6497, Lab No. D-52274, Retort Menber, Steer Creek, Lincoln County, Wyoming, (Shaldon, 1963, p. 217.)

12. Sample No. RAS 6497, Lab No. D-52274, Retort Menber, Steer Creek, Lincoln County, Wyouing, (Shaldon, 1963, P. 217.)

13. Sample No. VEM 72, Lab No. 6930, Retort Meabar, Coal Canyon, Linculn County, Wyoulng, (Mckalvey and others, 1953e, p. 11.)

14. Sample No. VFM 90, Lab No. 7375, Retore Member, Coal Canyon, Lincola County, Wyoning, (Mckelvey and others, 1953a, p, 11.)

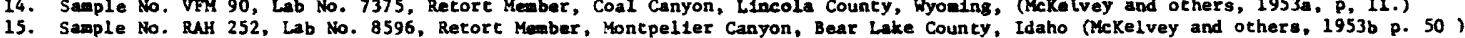

15. Sample No. RAH 252, Lab No. 8596, Retort Member, Montpeller Canyon, Bear Lake County, Idaho (McKelvey and othera, 1953b p. 50,
16. Sample No. RAH 246, Lab no. 8311, Retort Member, Hontpeller Canyon, Bear Lake County, Idaho (McKelvey and ochers, 1953b: p. S0.)

16. Sample No. RAH 246, Lab no. 8311, Retort Meaber, Hontpeller Canyon, Bear Lake County, Idaho (McKelvey and ochers, 1953b: p. S0.)
17. Sample No. LES 27, Lab No. 7260, Retort Member, Middle Fork P1ne Creek, LIncoln Councy, Wyoming (McKelvey and others, 1953a, p. 28.)

18. Sample No. LES 31, Lab No. 7264, Retort Member, Middle Fork PIne Creek, Lincoln County, Wyoming (Mckelvey and others, 1953a, p. 28)

19. Sample No. RAS 7204, Leb No. 90941, Recort Meaber, Deadilne RAdge, Lincoln Councy, Wyoulng (Sheldon, 1963, P. 253.)

20. Sample No. RAS 7208, Lab. No. 90945, Retort Meaber, Deadilne R1dge, Lincoln County, Wyoning (Sheldon, 1963, p. 253.)

21. Average quartz-ailicace of Retort Member of Phosphoria Formation.

22. Sample No. WRL 264, Leb No. 9579, Meade Peak Member, Daly Spur, Beaverhead County, Montana (Swanson and othera, 1953, P. 15.)

23. Sample WRL 265, Lab. No. 9580, Meade Peak Member, Daly Spur, Beaverhead County, Montana (Swanson and ochers, 1953, p. 15.)

24. Sample No. WRL 153, Lab. No. 9707, Meade Peak Member, B1g Sheep Canyon. Beaverheed County. Montana (Swanson and others, 1953, p. 29.)

25. Sample No. WRL 156, Lab. No. 9710, Meade Peak Member, B1g Sheep Canyon, Beaverhead County, Montana (Swanson and others, 1953, p. 29.)

26. Sample No. WOH 2716, Lab. No. 20174, Made Peak nember, Fort Hall, Bingnain County, Idano, (0' ralley and others, 1953, p. 5.)

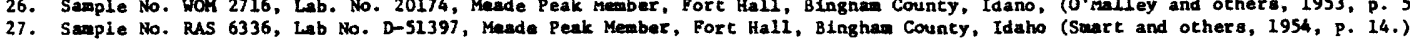

27. Sampie No. RAS 6336, Lab No. D-51397, Meade Peak Mewber, Fort Hall, Binghan County, Idaho (Smart and other s, 1954, P.
28. Sample No. RAS 6455, Lab No. D-52316, Maade Peak Meaber, Steer Creek, Lincoln County, Wyoming (Sheldon, 1963, p. 217.)

29. Sample MAW 6487, Lab. No. D-52284, Meade Peak Menber, Seeer Creek, Lincoln County, Wyouing (Sheldon, 1963, p. 217.)

30. Sample No. MAN 6425, Lab No. D-51964, Mande Peak Membar, Mud Springs, Cassia County, 'Idaho (Swart and others, 1954, P. 21.)

31. Sample No. RAS 6426, Lab. No. D-51963, Mende Peak Member, Mud Springs, Cassia County, Idato, (Smart and others, 1954. p. 21. )

32. Sample No. RAS 6432, Lab. No. D-51957, Meade Peak Member, Mud Springa, Casaia County, Idaho (Swart and others, 1954, p. 21.)

33. Sample No. RGW 6452, Lab No. D-51937. Mesde Perk Member, Hud Springs, Cassia County, Idaho (Smart and others, 1954, p. 20.)

34. Sample No. WOM 2974, Lab. Wo. 19814, Meade Penk Meaber, Swan Lake Gulch, Caribou County, Idaho (McKelvey and others, I953c., P. 44)

35. Sample No. WOM 2981, Lab. No. 19821, Meade Park Member, Swan Lake Gulch, Caribou County, Idaho (McKeivey and others, 1953e, p. 43.)

36. Salple No. HWP 4340, Lab No. 26612, Meade Peak Member, Rocky Canyon, Bannock County, Idaho (Dav1dson and others; 1953, p. I1.)

37. Sample No. RSJ 4331, Lab. No. 26669-F, Meade Peak Member, Rocky Canyon, Bannock County, Idaho (Dav1dson and others 1953, p. 10.)

38. Sample No. MET 4300, Lab. No. 45151, Meade Peak Meaber, Henry, Caribou County, Idaho (Davidson and others, 1953, p. 21.)

39. Sample No. RAS 4273, Lab. No. D-45128, Meade Peak Member, Henry, Caribou County, Idaho (Davidaon and others, 1953, p. 20.)

39. Sample No. RAS 4273, Lab. No. D-45128, Mesde Peak Member, Henry, Caribou County, Idaho (Davidaon and others, 1953, p. 20.)
40. Sample No. RPS 3626, Lab. No. 25057, Meade Peak Member, Cokevilie, Lincoln County, Wyoming (Sheldon and others, 1953, p. 43.)

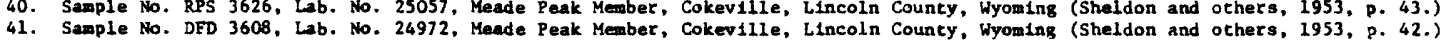

42. Sample No. LDC 7015, Lab. No. 88729, Meade Peak Member, M1ddle P1ney Lakes, Subletce County, Wyoming (Sheldon, 1963, p. 240.)

43. Sasple No. RPS 7027, Lab. No. 88704, Meade Peak Member, Middle PIney Lakes, Sublette County, Wyoming (Sheldon, 1963, p. 241.)

44. Sample No. RGW 3962, Lab. No. 25304, Meade Peak Member, North Crawford, Rich Councy, Utah, (Cheney and others, 1953, p. 9.)

45. Sample No. VEM 3936, Lab. No. 25260, Meade Peak Member, North Crawford, Rich County, Utah (Cheney and othere, 1953, p. 8.)

46. Semple No. MAW 6102, Lab. No. 51077, Maade Peak Member, Devils Slide, Morgan County, Uteh (Cheney end others, 1953, p. 14.)

47. Sample No. RAS 6165, Lab. No. D-51014, Meade Peak Memerer, Devils Slide, Morgan Councy, Utah (Cheney and othere, 1953, p. 12.)

48. Sample No. RAS 6191, Lab. No. D-51326, Meade Peak Meaber, Cumberland, Lineoln Councy, Wyoning (Sheidon, 1963, P. 267.)

49. Semple No. RGW 6201, Lab. No. D-51316, Made Peak Menber, Cumberland, Lincoln County, Wyouing, (Sheldon, 1963, p. 267.)

Sample No. MAW 6230, Lab. No. D-51287, Meade Peak Member, Cumberland, Lincoln County, Wyoming (Sheldon, 1963, P. 267.)

51. Sample No. MAN 6252, Lab. No. D-51265, Made Peak Member, CumberIand, Lincoln County, Wyon1ng (Sheldon, 1963, P. 267.)

52. Sample to. $\cos 2489$, Lab. No. 20921, Meade Peak Member, Wanrhodes Canyon, Utah County, Utah (Santh and others, 1952, p. 47.)

53. Sample No. DPS 2455, Lab. No. 20802, Meade Peak Member, Wanrhodes Canyon, Utah County, Uteh (Salth and othere, 1952, p. 46:)

54. Sample No. Gri 2340, Lab. No. 20823, Maade Peak Member, Wanrhodes Canyon, Utah Councy, Utah (Smith and othere, 1952, p. 43.)

55. Sample No. GFH 2570, Lab. No. 20868, Meade Peak Member, Wanthodes Canyon, Utah Councy, Utah (Smith and othera, 1952, p. 43.)

56. Sample No. RSS 2224 Lab. No. 20341, Meade Peak Member, R1ght Fk. Hobble Creek, Utah County, Utah (Sm1th and others, 1952, p. 40.)

57. Sample No. DPS 2381, Lab. No. D-8437, Meade Peak Member, R1ght Fk. Hobble Creek, Utah Councy, Utah (SEIth and others, 1952, p. 39.)

58. Sample No. MDS 2181, Lab. No. 20307, Meade Peak Member, Right Fk Hobble Creek, Utah County, Uteh (Smith and others, 1952, p.38.)

59. Sample No. LIS 2290, Lab. No. 20292, Meade Peak Meaber, R1ght Fk Hobble Creek, Utah County, Utah (Sa1th and others, 1952,

61. Sample No. JBC 2163, Lab to. 5315, Meade Peak Member, Wolf Creek, Wesecch County, Jkah (Smith and others, 1952, p. 25.) 


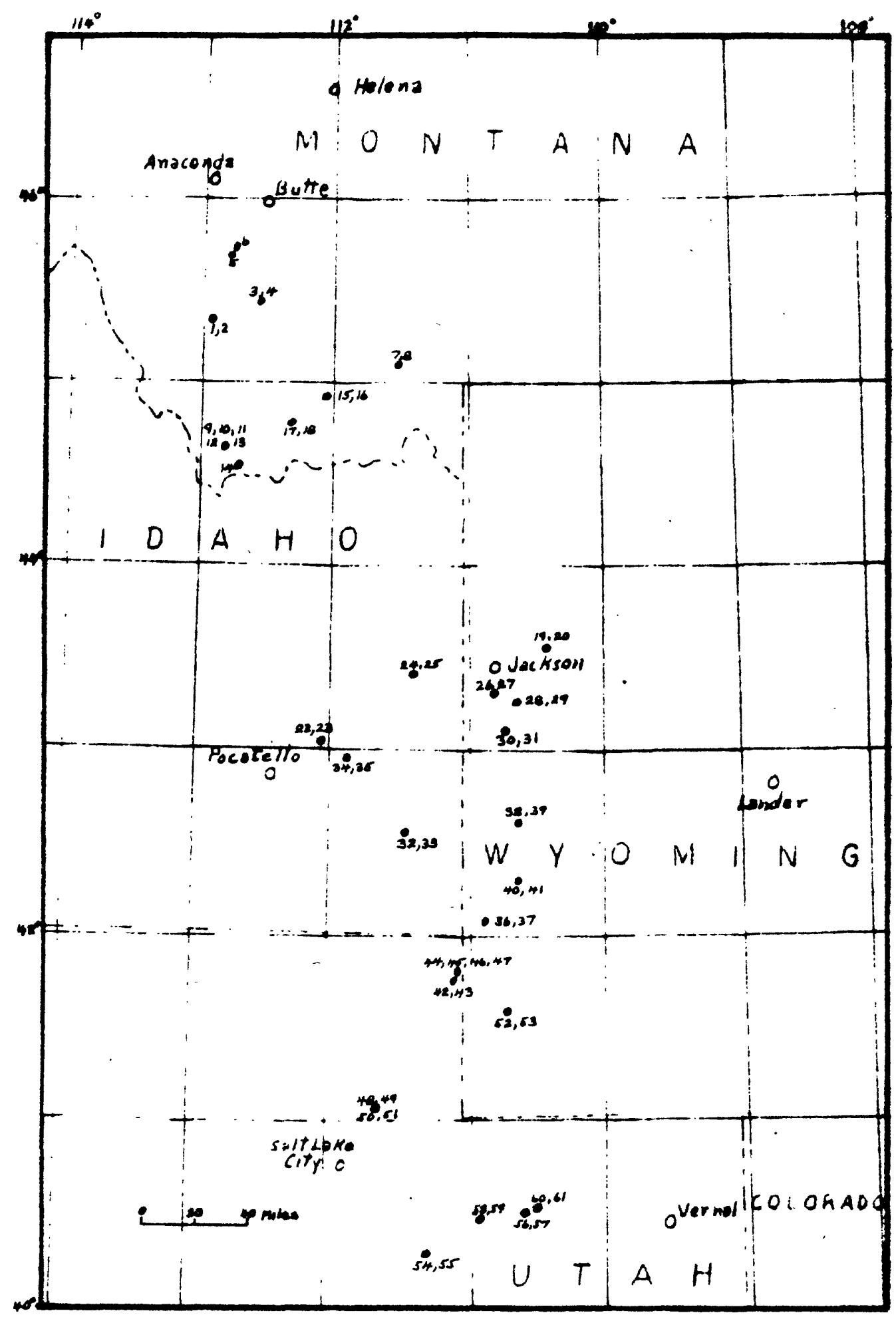

Figure 2.--Location of analyzed samples of carbonate rocks of table 2. Numbers are the analysis numbers. 
Table2 ${ }^{\prime}$.--Chemioal oomposition of the carbonate rocks of the Phosohoria Eormation

Retort Member

\begin{tabular}{|c|c|c|c|c|c|c|c|c|c|c|c|c|c|}
\hline & $\begin{array}{c}\text { RLP } 193 \\
1\end{array}$ & $\begin{array}{c}\text { DAB } 205 \\
2\end{array}$ & $\begin{array}{c}\text { JAP } 5430 \\
3\end{array}$ & $\begin{array}{c}\text { JAP } 5438 \\
4\end{array}$ & $\begin{array}{c}\text { JAP } 5423 \\
5\end{array}$ & $\begin{array}{c}\text { RFG } 5573 \\
6\end{array}$ & 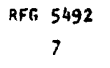 & $\begin{array}{c}\text { RFG } 5496 \\
8\end{array}$ & $\begin{array}{c}\text { WAL } 49 \\
9\end{array}$ & $\begin{array}{c}\text { WRL } 53 \\
10\end{array}$ & $\begin{array}{c}\text { WRL } 74 \\
11\end{array}$ & $\begin{array}{c}\text { WRL } 78 \\
12\end{array}$ & $\begin{array}{c}\text { WRL } 94 \\
13\end{array}$ \\
\hline $\mathrm{SiO}_{2}$ & 19.6 & 9.8 & 5.6 & 5.2 & 14.6 & 18.8 & 7.2 & 0.8 & 16.6 & 16.6 & 14.3 & 13.6 & 16.8 \\
\hline $\mathrm{Al}_{2} \mathrm{O}_{3}$ & 1.4 & 1.8 & 1.2 & 1.2 & 3.0 & 3.7 & 2.2 & 2.1 & 3.2 & 3.2 & 3.0 & 2.6 & 2.1 \\
\hline $\mathrm{Fe}_{2} \mathrm{O}_{3} \mathrm{~g}^{\prime}$ & .47 & .93 & .78 & .90 & 1.30 & 1.30 & 1.00 & 1.80 & $1.4 n$ & 1.70 & 1.50 & 1.40 & .96 \\
\hline Feo & .1 & .1 & .1 & .2 & .2 & .1 & .3 & .4 & .5 & .8 & .4 & .4 & .3 \\
\hline Mgo & 15.0 & 15.1 & 16.3 & 17.2 & 15.5 & 15.4 & 18.1 & 16.5 & 1.3 & 1.2 & 11.8 & 9.1 & 13.1 \\
\hline $\mathrm{CaO}$ & 24.7 & 29.4 & 30.6 & 30.0 & 25.7 & 23.0 & 26.6 & 26.7 & 38.9 & $39 \cdot 3$ & 28.3 & 31.6 & 27.1 \\
\hline $\mathrm{Na}_{2} \mathrm{O}$ & .09 & .16 & .12 & .12 & .37 & .11 & .10 & .17 & .12 & .12 & .26 & .32 & .17 \\
\hline $\mathrm{x}_{2} \mathrm{O}$ & .35 & .50 & .30 & .29 & .74 & .96 & .58 & .54 & .82 & .81 & .76 & .72 & .48 \\
\hline $\mathrm{H}_{2} \mathrm{O}^{\text {tot. }}$ & .50 & 2.00 & 1.22 & 1.04 & $\cdots$ & .80 & 1.214 & 2.00 & 2.625 & $2.60^{\mathrm{Cl}}$ & $2.86 \subseteq$ & 2.44 & $1.70^{\mathrm{c}}$ \\
\hline $\mathrm{H}_{2} \mathrm{O}^{-\mathrm{f}}-$ & .20 & $<.05$ & $<.05$ & $<.05$ & $\cdots$ & .20 & .10 & .30 & .3 & .3 & .1 & .2 & $\therefore .05$ \\
\hline $\mathrm{TiO}_{2}$ & .08 & .10 & .08 & .06 & .19 & .25 & .10 & .10 & .20 & .22 & .17 & .16 & .10 \\
\hline$P_{2} O_{5}$ & .1 .8 & 5.4 & 2.9 & 2.2 & .92 & .54 & .47 & 1.20 & .65 & .76 & 3.90 & $4.7 n$ & 1.20 \\
\hline Mno & .04 & .06 & .04 & .04 & .08 & .12 & .30 & .08 & .04 & .03 & .06 & .06 & .04 \\
\hline $\mathrm{CO}_{2}$ & 34.12 & 33.35 & 39.04 & $\cdots$ & 39.86 & 33.16 & 40.29 & 36.47 & 31.09 & 31.03 & 31.16 & 29.31 & 34.04 \\
\hline $\mathrm{SO}_{3}$ & $\cdots$ & $\cdots$ & $\cdots$ & $\cdots$ & $\cdots$ & $\cdots$ & $\cdots$ & $\cdots$ & $\cdots$ & $\ldots$ & .79 & $\cdots$ & .66 \\
\hline $\mathrm{Cl}$ & $-\cdots$ & $\cdots$ & $\ldots$ & $\ldots$ & $\cdots$ & $\cdots$ & $\ldots$ & $\cdots$ & $\cdots$ & $\cdots$ & $\cdots$ & $\cdots$ & $\ldots$ \\
\hline$F$ & .23 & .52 & .28 & .23 & $\cdots$ & .10 & .08 & .17 & .08 & .08 & .33 & .49 & .10 \\
\hline $\begin{array}{l}\text { Organigy } \\
\text { motter- }\end{array}$ & 1.0 & 1.3 & 1.5 & 1.3 & $\cdots$ & 1.3 & 2.4 & 3.7 & 3.4 & 2.9 & 3.5 & 3.6 & 3.1 \\
\hline 011 d & .20 & .10 & .10 & .13 & $\cdots$ & .18 & .12 & .23 & .59 & .50 & .42 & .50 & .40 \\
\hline$u$ & .001 & .001 & .002 & .002 & $\cdots$ & .001 & .001 & .001 & .001 & .001 & .001 & .002 & .001 \\
\hline U & $<.001$ & $<.001$ & .002 & $<.001$ & -- & .001 & $<. n 01$ & $<.001$ & .001 & .001 & $<.001$ & .002 & $<.001$ \\
\hline Ag & .0003 & .0003 & .0001 & .0001 & .0001 & .0003 & .0001 & .0001 & .0003 & .0001 & .0001 & .0001 & .0001 \\
\hline As & .006 & .002 & .004 & .003 & $\begin{array}{l}\text { wrong } \\
\text { sample }\end{array}$ & .001 & .005 & .003 & .001 & .002 & .002 & .004 & .003 \\
\hline$B$ & $<.005$ & $<.005$ & $<.005$ & $<.005$ & $<.005$ & $<.005$ & $<.005$ & $c .005$ & $<.005$ & $<.005$ & $<.005$ & $<.005$ & $<.005$ \\
\hline Qs & .003 & .003 & .001 & .001 & .01 & .01 & .003 & .003 & .01 & .01 & .01 & .01 & .003 \\
\hline $\mathrm{Cd}$ & $<.005$ & $<.005$ & $<.005$ & $<.005$ & $<.005$ & .03 & $<.005$ & $<.005$ & $<.005$ & $<.005$ & $<.005$ & $\because .005$ & $<.005$ \\
\hline $\mathrm{Cr}$ & .03 & .03 & .1 & .03 & .03 & .03 & .03 & .1 & .1 & .1 & .1 & .1 & .03 \\
\hline $\mathrm{Cu}$ & .003 & .003 & .003 & .003 & .003 & .003 & .001 & .003 & .003 & .203 & $.0 n 3$ &.$n 03$ & .003 \\
\hline Ga & $<.001$ & $<.001$ & $<.001$ & $<.001$ & .001 & $<.001$ & $<.001$ & $<.001$ & .001 & .001 & .001 & .001 & $<.001$ \\
\hline Mn & .01 & .01 & .01 & .01 & .03 & .03 & .1 & .03 & .01 & .01 & .03 &.$n 1$ & .01 \\
\hline Mo & $<.0005$ & .003 & .003 & .003 & .001 & .01 & .003 & .003 & .003 & .003 & .003 & .003 & $<.0005$ \\
\hline NI & .01 & .03 & .01 & .01 & .01 & .03 & .003 & .01 & .01 & .01 & .01 & .01 & .01 \\
\hline $\mathrm{Pb}$ & $<.001$ & $<.001$ & $<.001$ & $<.001$ & $<.001$ & .001 & .001 & .003 & $<.001$ & $<.001$ & $<.001$ & $<.001$ & $<.001$ \\
\hline sb & .0001 & .0002 & .0002 & .0002 & $\begin{array}{l}\text { wrong } \\
\text { samp le }\end{array}$ & $<.0001$ & .0001 & .0001 & .0001 & .0002 & .0001 & .0001 & .0002 \\
\hline Se & .0005 & $<.0005$ & $<.0005$ & $<.0005$ & $<.0005$ & $<.0005$ & $<.0005$ & $<.0005$ & $<.001$ & .001 & $<.0005$ & $<.0005$ & $\checkmark .0005$ \\
\hline Se & .0001 & .0001 & .003 & .001 & $\begin{array}{l}\text { wrong } \\
\text { sample }\end{array}$ & .003 & .001 & .0005 & .0005 & .0005 & .0003 & .0003 & .005 \\
\hline sr & .01 & .01 & .03 & .01 & .01 & .01 & .01 & .03 & .03 & .03 & .01 & .03 & .01 \\
\hline$v$ & .001 & $<.001$ & .003 & .003 & .003 & .001 & .oni & .003 & .003 & .003 & .001 & .001 & .003 \\
\hline $\mathbf{Y}$ & .003 & .01 & .001 & .003 & .001 & .001 & .001 & .003 & .003 & .003 & .01 & .01 & .001 \\
\hline ro & .0001 & .0003 & .0003 & .0001 & .0001 & .0001 & .0001 & .0003 & .0003 & .0003 & .0003 & .0003 & $<.00001$ \\
\hline In & .01 & .01 & .01 & $<.000$ & .01 & .03 & $<.008$ & $=.008$ &. .008 & $<.008$ & $<.008$ & $<.008$ & $<.208$ \\
\hline $2 r$ & $<.0008$ & .001 & .0008 & $<.0008$ & .001 & .003 & .0008 & .001 & .003 & .003 & .003 & .003 & .001 \\
\hline
\end{tabular}


Table 2'-.. Chemical composition of the arbonate rocks if the Phoaphoria Formation--Continued

Retort Herver--Continued

\begin{tabular}{|c|c|c|c|c|c|c|c|c|c|c|c|c|c|}
\hline & ERC 349 & ERC 683 & CWT 687 & CWT 673 & ERC 669 & MAW 4882 & RGW 4888 & Rve. $\frac{b f}{\text { Retort }}$ & wnM 2137 & HnP 2274 & RPS 5314 & RGW 5322 & RAS 4846 \\
\hline & 14 & 15 & 16 & 17 & 18 & 19 & 20 & 21 & 22 & 23 & $2 h$ & 25 & 26 \\
\hline $\mathrm{SiO}_{2}$ & 18.8 & 10.6 & 7.0 & 19.2 & 9.0 & 20.8 & 9.0 & 13.10 & 4.8 & 3.9 & 5.8 & 12.6 & 1.2 \\
\hline $\mathrm{Al}_{2} \mathrm{O}_{3}$ & 3.6 & 2.2 & 1.7 & 3.4 & 2.0 & 4.0 & 2.2 & 2.49 & .83 & 1.10 & 1.60 & 2.50 & .30 \\
\hline $\mathrm{Fe}_{2} \mathrm{O}_{3} \mathrm{I}^{\prime}$ & 2.20 & .98 & .78 & 1.40 & .76 & 2.30 & 1.40 & 1.26 & .30 & .41 & .53 & .82 & .17 \\
\hline Feo & .2 & .2 & .4 & .3 & .2 & .8 & .3 & .32 & .1 & .1 & .3 & .2 & .1 \\
\hline $\mathrm{MgO}$ & 5.8 & 16.2 & 15.0 & 13.3 & 14.9 & 12.8 & 16.8 & 13.02 & 17.0 & 16.0 & 14.8 & 14.0 & 18.1 \\
\hline coo & 32.0 & 27.7 & 30.8 & 22.9 & 28.0 & 21.8 & 27.6 & 28.64 & 30.8 & 32.4 & 30.5 & 29.3 & 31.1 \\
\hline $\mathrm{Na}_{2} \mathrm{O}$ & .20 & .11 & .17 & .12 & .14 & .16 & .23 & .17 & .12 & .11 & .48 & .72 & .08 \\
\hline $\mathrm{K}_{2} \mathrm{O}$ & .96 & .68 & .50 & .97 & .52 & 1.50 & .67 & .68 & .32 & .19 & .52 & .41 & .09 \\
\hline $\mathrm{H}_{2} \mathrm{O}^{\mathrm{tOt} .}$ & 1.72 & 1.20 & 1.12 & $2.72 \mathrm{~g}$ & $2.80^{\prime \prime}$ & 1.70 & 1.14 & 1.76 & .80 & -- & 1.92 & .84 & .90 \\
\hline $\mathrm{H}>\mathrm{O}^{-\mathrm{fl}}$ & .4 & .05 & .1 & .2 & .3 & $<.05$ & .1 & .15 & $<.05$ & $\cdots$ & .80 & .10 & .10 \\
\hline $\mathrm{riO}_{2}$ & .22 & .13 & .08 & .17 & .11 & .25 & .11 & .14 & .05 & .06 & $: 09$ & .15 & .03 \\
\hline$P . O_{5}$ & 2.40 & .59 & 460 & 1.00 & 1.50 & 1.20 & .39 & 1.91 & 2.2 & 3.6 & 5.2 & 2.5 & 2.3 \\
\hline MnO & .04 & .06 & .07 & .04 & .06 & .12 & .06 & .07 & .02 & .02 & .01 & .04 & .02 \\
\hline $\mathrm{CO}_{2}$ & 29.45 & 38.39 & 36.04 & 28.16 & 37.25 & 29.44 & 39.59 & 34.28 & 41.17 & -- & 34.18 & 35.45 & 42.09 \\
\hline $\mathrm{SO}_{3}$ & .32 & .34 & .62 & 1.08 & $\cdots$ & $\cdots$ & $\cdots$ & .635 & .31. & $\cdots$ & $\cdots$ & -- & -- \\
\hline $\mathrm{Cl}$ & $\cdots$ & $\cdots$ & $\cdots$ & $\cdots$ & $\cdots$ & --- & $-\cdot$ & $-\cdot$ & $\cdots$ & $\cdots$ & $\cdots$ & .03 & $\cdots$ \\
\hline$F$ & .27 & .08 & .42 & .15 & .15 & .15 & .08 & .21 & .18 & $\cdots$ & .52 & .23 & .24 \\
\hline organif/ & 1.4 & 1.8 & 1.9 & 4.0 & 3.2 & 3.1 & 1.9 & 2.4 & 1.7 & $\cdots$ & 3.5 & 1.9 & 2.7 \\
\hline 0 ild? & .15 & .23 & .10 & .75 & 1.00 & .45 & .05 & .33 & .14 & $\cdots$ & .10 & .20 & .12 \\
\hline$v$ & .001 & .001 & .002 & .001 & .001 & .001 & .001 & .0012 & .001 & $\cdots$ & .002 & .001 & .002 \\
\hline eU & .001 & $<.001$ & .002 & $<.001$ & .001 & .002 & $<.001$ & $\cdots$ & .001 & -- & .001 & .002 & .002 \\
\hline Ag & $<.00001$ & .0001 & .0001 & .0003 & .0003 & .00001 & .00001 & .0001 & .0001 & .2003 & .001 & .0003 & .0001 \\
\hline As & .003 & .006 & .003 & .002 & .004 & .002 & onl & .003 & .001 & & .004 & .004 & .008 \\
\hline$B$ & .005 & $<.005$ & $\because .005$ & $\because .005$ & $<.005$ & $\cdot .005$ & $<.005$ &. .05 & $<.005$ & $<.005$ & $c .005$ & $<.005$ & $<.005$ \\
\hline sa & .01 & .003 & .003 & .003 & .003 & .01 & .01 & $.003-.01$ & $.0 n 1$ & .001 & .001 & .001 & .201 \\
\hline $\mathrm{Cd}$ & $\because .005$ & $\because .005$ & $\therefore .005$ & $\cdots .005$ & .01 & $<.005$ & $<.005$ & $<.005$ & .01 & $<.005$ & $<.005$ & $<.005$ & .01 \\
\hline $\mathrm{Cr}$ & .1 & .03 & .03 & .1 & .03 & .1 & .01 & .03 & .03 & .03 & .03 & .03 & .03 \\
\hline Cu & .003 & .003 & .003 & .003 & .003 & .003 & .001 & .003 & .003 & .003 & .003 & .003 & .003 \\
\hline $\mathrm{Ga}$ & .001 & .001 & $\cdot .001$ & 4.001 & $<.001$ & .001 & $<.001$ & $<.001$ & $\therefore .001$ & .onl & .001 & $<.001$ & .001 \\
\hline Mn & .03 & .01 & 03 & .01 & .01 & .03 & .01 & .01 & .01 & .003 & .003 & .01 & .91 \\
\hline 40 & .003 & .003 & .001 & .003 & .003 & .01 & .003 & .003 & .0005 & $<.0005$ & .003 & .003 & .003 \\
\hline $\mathrm{Ni}$ & .03 & .01 & .01 & .01 & .003 & .01 & .003 & .01 & .01 & .01 & .03 & .01 & .03 \\
\hline $\mathrm{Pb}$ & $\cdot .001$ &. .001 & .001 & -.001 & $<.001$ & .001 & -.001 & .001 & 2.001 & $<.001$ & $<.001$ & $\cdot .001$ & -.001 \\
\hline Sb & .0002 & .0002 & .0002 & .0002 & .0001 & .0001 & $<.0001$ & .00015 & .0001 & & .0005 & .0005 & .001 \\
\hline Sc & .001 & .0005 & $<.0005$ & $<.0005$ & .0005 & .0005 & $<.0005$ & $<.0005$ & $<.0005$ & $<.0005$ & .0005 & $<.0003$ & .0005 \\
\hline Se & .001 & 005 & .0007 & .001 & .001 &.$\infty 07$ & .0003 & .00142 & .005 & & .003 & .005 & .0003 \\
\hline $\mathrm{sr}$ & .03 & .01 & .03 & .03 & .03 & .01 & .01 & .01 & .03 & .01 & .03 & .01 & .01 \\
\hline$v$ & .003 & .001 & .003 & .003 & .01 & .001 & $\ldots 001$ & .003 & .003 & .003 & .01 & .003 & .003 \\
\hline$r$ & .003 & .001 & .01 & .003 & .003 &.$\infty 03$ & $<.001$ & .003 & .003 & .003 & .003 & .201 & .001 \\
\hline Yb & .0003 & $<.00001$ & .0003 & .0001 & .0001 & .0001 & $<.00001$ & .0003 & .0001 & .0003 & .0001 & $<.00001$ & $<.00001$ \\
\hline $2 n$ & .01 & .01 & .01 & .01 & .01 & $\therefore .008$ & $<.008$ & $<.008$ & .01 & $<.008$ & .03 & .01 & .03 \\
\hline $2 r$ & .003 & .0003 & .0008 & .001 & .001 & .003 & $<.0008$ & $<.0008-.003$ & $<.0008$ & $<.0008$ & $<.0008$ & .001 & $<.0008$ \\
\hline
\end{tabular}




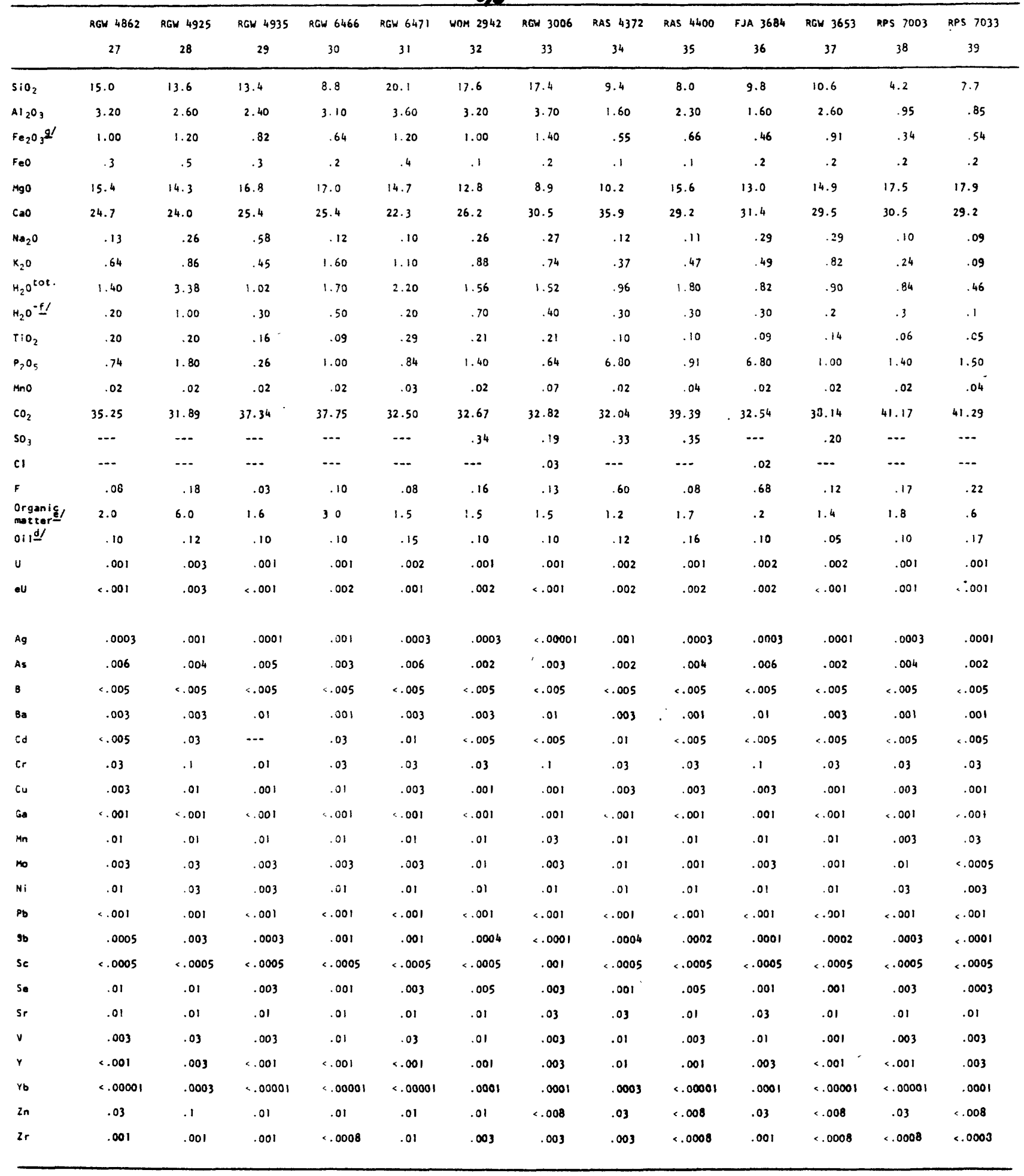


Table2'. - Chemical composition of the carbonate rocks of the Phosphoria Eormation--Conti it

Meade Peak Member--Cont inued

\begin{tabular}{|c|c|c|c|c|c|c|c|c|c|c|c|c|}
\hline & $\begin{array}{c}\text { RAS } 7095 \\
40\end{array}$ & $\begin{array}{c}\text { ERC } 7105 \\
41\end{array}$ & $\begin{array}{l}\text { VEH } 141 \\
42\end{array}$ & $\begin{array}{l}\text { VEM } 120 \\
43\end{array}$ & $\begin{array}{c}\text { RGW } 3953 \\
44\end{array}$ & $\begin{array}{c}\text { Rfit } 3976 \\
45\end{array}$ & $\begin{array}{c}\text { RA5 } 3899 \\
46\end{array}$ & $\begin{array}{c}\text { RAS } 3895 \\
47\end{array}$ & $\begin{array}{c}\text { HAW } 6096 \\
48\end{array}$ & $\begin{array}{c}\text { HAW } 6082 \\
49\end{array}$ & $\begin{array}{l}\text { RAS } 6160 \\
50\end{array}$ & $\begin{array}{c}\text { TMC } 6148 \\
51\end{array}$ \\
\hline $\mathrm{SlO}_{2}$ & 14.3 & 20.8 & 16.0 & 19.7 & 16.2 & 18.1 & 3.4 & 7.4 & 12.5 & 6.6 & 13.9 & 22.2 \\
\hline $\mathrm{Al}_{2} \mathrm{O}_{3}$ & 2.70 & 4.20 & 1.20 & 1.00 & .86 & 2.60 & 2.00 & 2.90 & 2.80 & 1.80 & 1.60 & 1.10 \\
\hline $\mathrm{Fe}_{2} \mathrm{O}_{3} \mathrm{~g}^{\prime}$ & .90 & 1.60 & .46 & .50 & .36 & .68 & 1.50 & .68 & 1.20 & .78 & .66 & .54 \\
\hline FEo & .3 & .3 & .1 & .2 & .2 & .3 & .1 & .1 & .1 & .2 & .2 & .2 \\
\hline H9O & 13.6 & 11.5 & 2.5 & 4.1 & .76 & .79 & .96 & .85 & 16.80 & 17.00 & 12.10 & 9.40 \\
\hline $\mathrm{CaO}$ & 27.2 & 23.3 & 42.8 & 37.8 & 44.3 & 41.4 & 46.5 & 46.4 & 25.4 & 30.0 & 31.4 & 30.6 \\
\hline Na, 0 & .12 & .15 & .09 & .10 & .08 & .18 & .12 & .13 & .28 & .10 & .14 & .10 \\
\hline$k_{2}=0$ & .62 & 1.20 & .28 & .22 & .14 & .55 & .11 & .60 & .70 & .39 & .30 & .19 \\
\hline$H: 0^{t 0 t .}$ & 2.20 & 2.50 & 94 & .60 & .60 & .96 & 1.16 & 1.24 & 1.26 & .82 & .66 & .80 \\
\hline $4,0^{-f}-$ & .7 & 8 & .7 & .1 & .1 & .2 & $<.05$ & .2 & $<.05$ & .05 & .4 & 2 \\
\hline $\mathrm{NO}_{2}$ & .20 & .28 & .06 & .06 & .04 & .16 & .11 & .08 & .14 & .10 & .08 & .06 \\
\hline$P, O_{5}$ & 2.10 & 1.60 & .94 & 1.00 & 1.00 & 2.40 & 2.60 & 2.40 & .72 & 2.20 & 1.00 & .26 \\
\hline $\mathrm{MnO}$ & .02 & .04 & .01 & .02 & .01 & .02 & .04 & .02 & .02 & .02 & .02 & .02 \\
\hline $\mathrm{CO}_{?}$ & 33.26 & 28.63 & 34.66 & 33.56 & 34.58 & 30.37 & 34.85 & 34.77 & 37.31. & 39.34 & 36.36 & 32.51 \\
\hline $\mathrm{SO}_{3}$ & -- & $--:$ & $\cdots$ & .31 & $\cdots$ & .32 & $\cdots$ & $\ldots$ & $\cdots$ & -.. & $\ldots$ & $\cdots$ \\
\hline $\mathrm{Cl}$ & --- & $\cdots$ & $\cdots$ & $\cdots$ & $\cdots$ & $\cdots$ & $\cdots$ & $\cdots$ & $\cdots$ & $\cdots$ & $\cdots$ & $\cdots$ \\
\hline$F$ & .23 & .18 & .11 & .12 & .11 & .32 & .25 & .24 & .10 & .24 & .12 & .11 \\
\hline Organic/ & 2.4 & 3.3 & 1.2 & 1.3 & .7 & 1.1 & 1.3 & 1.4 & 1.2 & 2.1 & 1.3 & 1.1 \\
\hline $0: 1 d$ & .13 & .16 & .14 & .12 & .13 & .12 & .13 & .12 & .10 & .10 & .12 & .13 \\
\hline $\mathrm{U}$ & .002 & .001 & .001 & .001 & .001 & .002 & .001 & .001 & .001 & .001 & .001 & .001 \\
\hline ev & .001 & .002 & -.001 & $<.001$ & $<.001$ & .001 & $<.001$ & $<.001$ & .001 & .001 &. .001 & .001 \\
\hline Ag & .0003 & .001 & .00003 & .0001 & .0001 & .0003 & .0003 & .0003 & .0001 & .0001 & .0001 & .0003 \\
\hline As & .002 & .001 & .002 & $<.001$ & .001 & .002 & .003 & .003 & $\cdot .001$ & .004 & $\therefore .001$ & .001 \\
\hline$B$ & $<.005^{\circ}$ & $\because .005$ & $<.005$ & $<.005$ & $<.005$ & $<.005$ & $<.005$ & $<.005$ & $=.005$ & .005 & .005 & .005 \\
\hline $8 a$ & .01 & .01 & .003 & .01 & .003 & .01 & .01 & .01 & .003 & .003 & .01 & .003 \\
\hline $\mathrm{Cd}$ & $<.005$ & .005 & $<.005$ & .005 & $<.005$ & .005 & $\therefore .005$ & $c .005$ & $=.005$ & $\cdot .005$ & .005 & .005 \\
\hline $\mathrm{Cr}$ & .1 & .1 & .03 & .03 & .03 & .1 & .1 & .1 & .03 & .1 & .1 & .03 \\
\hline Cu & .003 & .01 & .001 & .001 & .003 & .003 & .003 & .01 & .001 & .003 & .003 & .003 \\
\hline Ga & .201 & .001 & $<.001$ & $<.001$ & $<.001$ & .001 & $<.001$ & $<.001$ & $\because .001$ & .001 & $<.001$ & $\therefore .001^{\prime}$ \\
\hline Mn & .01 & .01 & .003 & .01 & .003 & .01 & .03 & .01 & .01 & .01 & .01 & .01 \\
\hline Ho & .003 & .01 & $<.0005$ & $<.0005$ & $<.0005$ & $<.0005$ & .003 & .003 & .0005 & .003 & $=0005$ & $\times .0005$ \\
\hline $\mathrm{Ni}$ & .03 & .01 & .003 & .003 & .003 & .01 & .03 & .01 & .003 & .01 & .003 & .003 \\
\hline $\mathrm{Pb}$ & $<.001$ & .001 & $<.001$ & $\therefore .001$ & $\times .001$ & $<.001$ & -.001 & $<.001$ & $\cdots .001$ & .001 & .001 &. .001 \\
\hline Sb & .0007 & .0005 & .0002 & .0001 & $<.0001$ & .0002 & .0003 & .0005 & .0001 & .0003 & .0001 & .0001 \\
\hline Sc & .0005 & .0005 & .0005 & $<.0005$ & .0005 & .001 & .0005 & .0005 & .0005 & .0005 & .0005 & .0005 \\
\hline S. & .001 & .003 & .0003 & .0003 & .0001 & .0003 & .003 & .0003 & .001 & .001 & .005 & .001 \\
\hline sr & .01 & .01 & .03 & .03 & .03 & .03 & .1 & .03 & .01 & .03 & .03 & .01 \\
\hline$v$ & .01 & .03 & $<.001$ & $<.001$ & .001 & .003 & .01 & .003 & $<.001$ & .001 & .001 & .001 \\
\hline$y$ & .001 & .003 & .003 & .001 & .001 & .01 & .01 & .003 & .001 & .003 & .001 & .001 \\
\hline$r_{b}$ & .0001 & .0003 & .0001 & $<.00001$ & .0001 & .0003 & .0003 & .0003 & $<.00001$ & .0001 & -.00001 & .00001 \\
\hline $2 n$ & .01 & .03 & $<.008$ & .008 & $<.008$ & .008 & .01 & .008 & $\therefore .008$ & $c .008$ & .008 & .008 \\
\hline $2 r$ & .003 & .01 & .001 & .001 & .001 & .003 & .001 & .0008 & $<.0008$ & .0008 & .001 & $\therefore .0008$ \\
\hline
\end{tabular}


Table2의... hemisal composition the sarbmats rocks of the Phosphoria Formation--Continued

Heade Peak Member--Continued

\begin{tabular}{|c|c|c|c|c|c|c|c|c|c|c|c|c|}
\hline & $\begin{array}{l}\text { RGW } 6195 \\
52\end{array}$ & $\begin{array}{c}\text { TMC } 62 i 1 \\
53\end{array}$ & $\begin{array}{c}\text { RSS } 2241 \\
54\end{array}$ & $\begin{array}{c}\text { GFH } 2313 \\
55\end{array}$ & $\begin{array}{l}\text { JWH } 2058 \\
56\end{array}$ & $\begin{array}{c}\text { JWH } 2063 \\
57\end{array}$ & $\begin{array}{c}\text { JWH } 2005 \\
58\end{array}$ & $\begin{array}{l}\text { JWH } 2008 \\
59\end{array}$ & $\begin{array}{l}\text { JWH } 2097 \\
60\end{array}$ & $\begin{array}{l}\text { JWH } 2109 \\
61\end{array}$ & $\begin{array}{l}\text { Ave. } 5 \text { I } \\
\text { Meade } \\
\text { Peak } \\
62\end{array}$ & $\begin{array}{l}\text { Ave. } \frac{b}{} \\
\text { Phosphoria } \\
63\end{array}$ \\
\hline $\mathrm{SiO}_{2}$ & 10.1 & 12.0 & 19.9 & 11.2 & 15.1 & 15.6 & 9.8 & 14.8 & 19.9 & 17.1 & 12.64 & 12.79 \\
\hline $\mathrm{Al}_{2} \mathrm{O}_{3}$ & 1.40 & 2.80 & 2.80 & 1.00 & 1.40 & 3.00 & .42 & 1.60 & 1.60 & 1.50 & 2.01 & 2.17. \\
\hline $\mathrm{Fe}_{2} \mathrm{O}_{3} \mathrm{~g}^{\prime}$ & 1.00 & 1.80 & $1.40^{\circ}$ & .86 & 1.10 & 1.00 & .38 & .97 & 1.20 & .78 & .83 & .98 \\
\hline FeO & .1 & .7 & $\cdot 3$ & .3 & .3 & .3 & .1 & .2 & .5 & .3 & .23 & .26 \\
\hline $\mathrm{MgO}$ & 15.70 & 12.00 & .71 & 1.00 & 16.00 & 15.50 & 15.4 & 15.9 & 15.0 & 16.2 & 12.07 & 12.38 \\
\hline $\mathrm{CaO}$ & 28.4 & 23.6 & 39.4 & 46.4 & 25.5 & 24.8 & 31.3 & 26.0 & 25.0 & 24.4 & 31.00 & 30.22 \\
\hline $\mathrm{Na}_{2} \mathrm{O}$ & .32 & .40 & .14 & .15 & .13 & .18 & .12 & .15 & .16 & .12 & .19 & .18 \\
\hline $\mathrm{K}_{2} \mathrm{O}$ & .43 & .68 & .74 & .19 & .37 & .76 & .09 & .37 & .49 & .40 & .50 & .56 \\
\hline$H_{2} \mathrm{O}^{\text {tot. }}$ & .68 & 4.50 & 1.76 & .56 & -.. & 1.36 & .50 & .70 & .92 & 1.48 & 1.30 & 1.45 \\
\hline $\mathrm{H}_{2} \mathrm{O}^{-\mathrm{f}} \mathrm{I}$ & .1 & 1.7 & .2 & -.05 & $\cdots$ & .3 & -05 & .05 & .2 & .2 & .32 & .26 \\
\hline $\mathrm{TiO}_{2}$ & .08 & .20 & .12 & .04 & .28 & .14 & .03 & .08 & .08 & .08 & .11 & .12 \\
\hline P.O. & 4.20 & 4.00 & 2.40 & 4.10 & 1.40 & 2.00 & .24 & $\therefore .60$ & 1.00 & .92 & 2.02 & 1.99 \\
\hline MnO & .04 & .04 & .02 & .00 & .02 & .01 & .02 & .02 & .02 & .01 & .02 & .04 \\
\hline $\mathrm{CO}_{2}$ & 35.13 & 27.25 & 28.59 & 34.10 & $\cdots$ & 33.52 & 41.30 & 36.11 & 33.68 & 36.13 & 35.10 & 34.83 \\
\hline $\mathrm{SO}_{3}$ & -.. & $\cdots$ & .54 & $\cdots$ & & $\cdots$ & .16 & $\cdots$ & .54 & .53 & .343 & .441 \\
\hline Cl & $\cdots$ & $\cdots$ & $\cdots$ & $\cdot-\cdot$ & $\cdots$ & .03 & -- & $\cdots$ & $\cdots$ & $\cdots$ & $\cdots$ & $\cdots$ \\
\hline$F$ & .38 & .35 & .23 & .34 & $\cdots$ & .22 & .04 & .18 & .13 & .12 & .21 & .21 \\
\hline $\begin{array}{l}\text { Organic, } \\
\text { malcere }\end{array}$ & 2.2 & 7.7 & 2.0 & .8 & $\cdots$ & 1.8 & .7 & 2.0 & 1.6 & 1.9 & 1.92 & 2.09 \\
\hline $0 \mathrm{il}{ }^{d \prime}$ & .20 & .10 & .13 & .12 & -- & .13 & .10 & .12 & .14 & .10 & .12 & .19 \\
\hline v & .001 & .003 & .002 & .001 & $\cdots$ & .002 & .001 & .001 & .001 & .001 & .0014 & .0013 \\
\hline eu & $\because .001$ & .005 & .002 & $<.001$ & $-\cdot$ & .001 & .001 & .001 & .001 & $<.001$ & -- & +- \\
\hline Ag & .0003 & .0003 & .0001 & $<.00001$ & .001 & .0001 & .0003 & .0001 & $<.00001$ & .0001 & .0003 & .0001 \\
\hline As & .001 & .001 & .002 & .001 & & .005 & .002 & .006 & .001 & .002 & .00281 & .00288 \\
\hline B & .005 & $<.005$ & $<.005$ & $c .005$ & $<.005$ & $<.005$ & $<.005$ & $<.005$ & $<.005$ & .005 &. .005 & $=.005$ \\
\hline Ba & .003 & .01 & .01 & .003 & .003 & .003 & $<.0005$ & .003 & .003 & .003 & .003 & .003 \\
\hline $\mathrm{Cd}$ & $<.005$ & .01 & $<.005$ & $<.005$ & $<.005$ & $<.005$ & $<.005$ & $<.005$ & $<.005$ & $<.005$ & .005 & $<.005$ \\
\hline$c r$ & .03 & .1 & .1 & .1 & .03 & .1 & .003 & .03 & .03 & .03 & 03 & .03 \\
\hline $\mathrm{Cu}$ & .003 & .01 & .003 & .001 & .003 & .003 & .001 & .001 & .003 & .01 & .003 & .003 \\
\hline Ga & $<.001$ & $<.001$ & .001 & $<.001$ & $<.001$ & $<.001$ & $<.001$ & $<.001$ & $<.001$ & $<.001$ & $<.001$ & .001 \\
\hline $4 n$ & .01 & .01 & .01 & .003 & .003 & .003 & .01 & .01 & .01 & .01 & .01 & .01 \\
\hline Mo & $<.0005$ & .03 & $<.0005$ & .0005 & .001 & $<.0005$ & $<.0005$ & .001 & $<.0005$ & $<.0005$ & .0005 & .003 \\
\hline $\mathrm{Ni}$ & .03 & .03 & .01 & .003 & .003 & .01 & .003 & .01 & .003 & .003 & .01 & .01 \\
\hline $\mathrm{Pb}_{b}$ & $<.001$ & .001 & $<.001$ & $<.001$ & .001 & $<.001$ & .001 & .003 & $<.001$ & $<.001$ & $<.001$ & $<.001$ \\
\hline so & $<.0001$ & .001 & .0001 & $<.0001$ & & .0005 & $<.0001$ & .0001 & .0001 & $<.0001$ & .00039 & .00020 \\
\hline Sc & $<.0005$ & $<.0005$ & $<.0005$ & $<.0005$ & $<.0005$ & $<.0005$ & .0005 & $<.0005$ & $<.0005$ & $<.0005$ & $<.0005$ & $<.0005$ \\
\hline Se & .001 & .002 & .001 & .0007 & . & .0003 & .0005 & .0003 & .002 & .0003 & .00221 & .002 \\
\hline sr. & .01 & .01 & .03 & .03 & .01 & .01 & .003 & .01 & .01 & .01 & .01 & .01 \\
\hline$v$ & -.001 & .03 & .003 & $<.001$ & $\because .001$ & $=.001$ & $<.001$ & .001 &. .001 & $<.001$ & .003 & .003 \\
\hline$r$ & .001 & .01 & .01 & .003 & .001 & .001 & $<.001$ & $<.001$ & .001 & .001 & .003 & .003 \\
\hline$Y_{b}$ & .0001 & .0003 & .0003 & .0001 & .00001 &. .00001 & .00001 & .00001 & $<.00001$ & $<.00001$ & $<.00001$ & $<.00001$ \\
\hline In & .03 & .03 & $<.008$ & $<.008$ & .03 & .01 & .03 & .03 & .008 & .01 & $<.008$ & .008 \\
\hline $2 r$ & .001 & .003 & .001 & $\cdot .0008$ & .0008 & .0008 & .0008 & $<.0008$ & .0008 & $<.0008$ & $<.0008$ & $<.0008$ \\
\hline
\end{tabular}




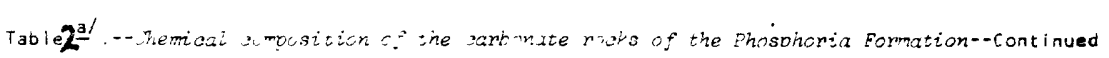

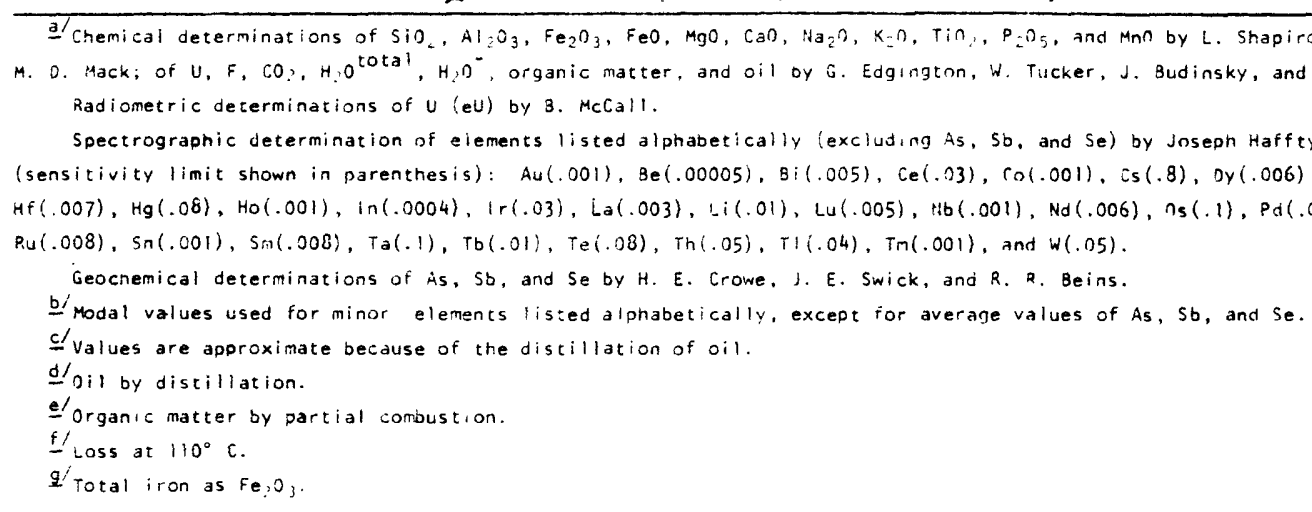

1. Sample No. RLP-193, Lab. No. 140538. Fetort Member, Selly Gulch, Beaverhead County. Montana (Cressman and Swanson, 1954. D. 459).

2. Sample NO. OAB-205, Lab. No. '40542, Retort Member, Kelly Gulch. Beaverhead County, Montana (Cressman and Swanson, 1964, 0.438 ).

3. Sample No. JAP 5430, Lab. No. 053316, Retort Member. North 8ig Hole Canyon, Madison County, Montana (Cressman and Swanson. 1064, p. 537).

4. Sample No. JAP 5438, Lab. No. 053308, Retort Member. North Big Hole Canyon, Macison County, Montana (Cressiman and Swanson, 1964, P. 537).

5. Sample No. JAP 5423, Lab. No. 150106, Retort Member, Canvon Creek 3. Beaverhear rounty, Mantana (Cressman and Swanson, 1964, D. 541).

7. Sample No. RFG 5492, Lab. No. 140480, Retort Member, Indian Creek, Beaverhead County. Montana (Cressman and Swanson, 1964, 0.545 ).

8. Sample No. RFG 5496, Lab. No. 140554, Retort tember, Indian Creek. Beaverhead County, Montana (Cressman and Swanson, 1964, p. 544).

9. Sample No. WRL -49. Lab. No. 9604, Retort Member, Hidden Pasture Creek, Beaverhead County, Montana (Swansmn and others, 1953, $p$. 27)

10. Sample No. WRL 53, Lab. No. 9608, Retort Member, Hidden Pasture Creek, Beavernead County, Montana (Swanson and others, 1953, $p$. 27).

11. Sample No. WRL 74, Lab. No. 9629, Retort Member. Hidden Pasture Creek, Beaverhead Count\%, Montana (Swanson and others, 1953, p. 26).

12. Sample No. WRL 78, Lab. No. 9633, Retort Member. Hidden Pasture Creek, Beaverhead County, Montana (5wanson and nthers, 1953, g. 26).

13. Sample No. WRL 94, Lab. No. 9649. Retort Member. Hidden Pasture Creek, Beaverhead County. Montana (Swanson and others, 1953, $p$. 26).

14. Sample 1o. ERC 349, Lab. No. 25455, Ketort Member, Little Sheep Creek, Beaverhead County, Montana (Cressman and Swanson, 1964, o. 477)
15. Sample No. ERC 683, Lab. No. 25706, Retort Member, Sliderock Mountain, Madison County, Montana (Cressman and Swanson, 1964, p. 498).

15. Sample No. ERC 683, Lab. No. 25706. Retort Member, Sliderock Mountain, Madison County, Montana (Cressman and Swanson, 1964, $p .4987$ ).
16 . Sample Ho. CWT 637, Lab. No. 25691, Retort Merber, sliderock. Mountain, Madi son County. Inntana (Cressman and Swanson, 1964, $p$. 497)

17. Sample Ho. CWT 673, Lab. No. 25722, Retort :tember, West Fork of Blacktail Creek, Beaverhend Caunty. Montana (Cressman and others, 1953, p. II).

13. Sample No. ERC 669, Lab. No. 25744, Retort Member, liest fork of Blacktail Creek, Beavernead County, Montana (Cressman and others, 1953. p. 11).

19. Sample !o. MAW 4882, Lab. Mo. 045450 , Retort terber, Crystal Creek, Teton County, Hyomina (Sheldon, 1963, p. 187).

20. Sample No. RGW 4888, Lab. No. 045444, Retort Member, Crystal Creek, Teton County, Jyoming (Sheldon, 1963, p. 187).

21. Average carbonate of Retort Member of Phosphoria Format: in.

22. Sample No. WOM 2137. Lab. No. 20181, Meade Peak Member, Fort Hail, Bingham County, Idaho (nintalley and others, 1353, p. 6).

23. Sample No. WOM 2274, Lab. No. 150105. Meade Peak Member. Fort Hali, Bingham County, Idaho (n'Malley and others, 1953, 0.6$)$.

24. Sample No. RPS 5314, Lab. No. 77845, Meade Peak Member. Fall Creek, Onnneville County, Idahn (Smart and others, 1954, P. 6).

26. Sample No. RAS 4846, Lab. No. D45420, Meade Peak Member, Hoback, Teton County, Wyominn (Sheldon, 1963, p. 207).

27. Sample No. RGW 4862, Lab. No. 045404, Meade Peak Member, Hoback, Teton County, lyoming. (sheldon, 1963, p. 207).

28. Sample No. RGW 4925, Lab. No. 046052, Meade Peak Member, Buck Creek, Teton County, Wyóming (5heldon, 1963, p. 211).

29. Sample No. RGW 4935, Lab. No. 046042, Meade Peak Member, Buck Creek, Tetnn Councy, Wyoming (Sheldon, 1963, p. 211).

30. Sample No. RGW 6466, Lab. No. 052305, Meade Peak Menbar, Steer Creek, Lincoln Councy. Wyoming (5heldon, 1963, p. 217).

32. Sample No. WOM 2942, Lab. No. 18782, Meade Peak Hember, Swan Lake Guich, Caribou County, Idano (Mckelvey and others, ig53, p. 44).

33. Sample No. RGW 3006, Lab. No. 19748, Meade Peak Member, Swan Lake Gulch, Caribou County, idann (Itckelvey and others, 1953, 0.42 ).

33. Sample No. RAS 4372, Lab. No. 26650, Meade Peak Merber, Rocky Canynn, Rannock County, Idaho (Davidson and nthers, l953, 0 . Ii).

35. Sample No. RAS 4400 , Lab. No. 26ń62. 'leade Peak Member, Rockv Canyon, Bannock Councy, Idaho (navidson and others, 1953, D. 11).

36. Samole No. FJA 3084, Lab. No. 24981, leade Peak Meriber. Cakeville, Lincoln County, Wyoming (5heldon and others, ig53, p. 45).

37. Sample No. RGW 3653, Lab. No. 25027 , Meade Paak Member, Cokevilie, Lincoln County, Wyoming (Sheldon and others, 1953, p. 43).

39. Sample No. RPS 7033, Lab. No. 88710, Meade Peak Member, Middle Piney Lakes, Sublette County, 14yoming (Sheldon, 1963, p. 240).

40. Sample No. RAS 7095, Lab. No. 38612, Meace Peak Member, Fontenelle Creek, Lincoln County, Wyomina (Sheldon, 1963, p. 250).

41. Sample No. ERC 7105, Lab. No. 88622, Meade Peak Member, Fontenelle Creek, Lincoln County, iyomina (Sheldon, 1963, p. 250).

42. Sample No. VEM 141, Lab. No. 7/43, Meade Peak Mermer, Brazer Canyon, Rich County, Utah (Smith and others, 1952, $p .10)$.

43. Sample No. VEM 120, Lab. No. 7122, Meade Peak Member, Brazer Canyon, Rich County, Utah (Smith and others, 1952, $p .9$ ).

44. Sample Ho. RGW 3953, Lab. No. 25295, Meade Peak Member, North Crawford, Rich County, Utan (Cheney and nthers, 1953, p. 9).

45. Sample No. RGW 3976, Lab. No. 25318. "leade Peak Member, North Crawford, Rich County, Utah (Cheney and others, 1953, 0. 8).

46. Sample No. RAS 3899, Lab. No. 25235. Meade Peak Member, Nortin Crawford, Rich County, Utah (Cheney and others, 1953, p. 8)

47. Sample No. RAS 3895, Lab. No. 25231, Meade Peak Member, North Crawford, Rich County, Utah (Cheney and others, 1953, 0.8$)$.

48. Sample No. MAW 6096, Lab. No. 051083, Meade Peak Member. Devils Slide, Morqan County, Utah (Cheney and others, 1953. p. 14).

49. Sample No. MaW 6082, Lab. No. 051097. Meade Peak Member, Devils S1ide, Morgan County, Utah (Cheney and others, 1953. p. 15).

50. Sample No. RAS 6160. Lab. No. 051019, Meade Peak Member, Devils Slide, Morgan County, Utah (Cheney and others, 1953, p. 12).

51. Sample No. TMC 6148, Lab. No. 051031, Meade Peak Member, Devils S1ide, Marqan County, Utah (Cheney and nthers, 1953, 0.12 ).

52. Sample No. RGW 6195, Lab. No. 051322, Meade Peak Member, Cumberland, Lincoln County, Mynming (Sheldon, 1963, p. 267).

53. Sample No. TMC 62\%1, Lab. No. D51306, Meade Peak Member, Cumberland, Lincoln County, Wyoming (Sheldon, 1963, p. 267).

54. Sample No. RSS 2241, Lab. No. 20358. Meade Peak Member, Right Fork of Hobble Creek, Iltan County, Utah (Smith and athers, 1952. p. 40).

55. Sample No. GFH 2313, hab. No. 20264, Meade Peak Member, Rignt Fork of Hobble Creek. Utah County. Utan (Smith and others, 1952, p. 37).

56. Sample No. JWH 2057. Lab. No. 150107, Meade Peak Member, Dry Canyon, Ouchesne County, Utah (Smith and others, 1952, P. 30).

57. Sample No. JWH 2063, Lab. Mo. 5117. Meade Peak Member, Ory Canyon, Duchesne County, Utah (Smith and others, 1952. p. 30).

58. Sample No. JWH 2005. Lab. No. 5326, Meade Peak Member, Wolf Creet., Wasatch County, Utah (Smith and nthers, 1952, p. 26).

59. Sample No. JWH 2008, Lab. No. 5329, Meade Peak Memoer, Wolf Creek, Wasatch County, Utah (Smith and others, 1952, p. 26).

61. Sample No. JWH 2109, Lab. No. 6790, Meade Peak Member, Lake Fork, Duchesne County, Utah (Smith and others, 1952, p. 32). 


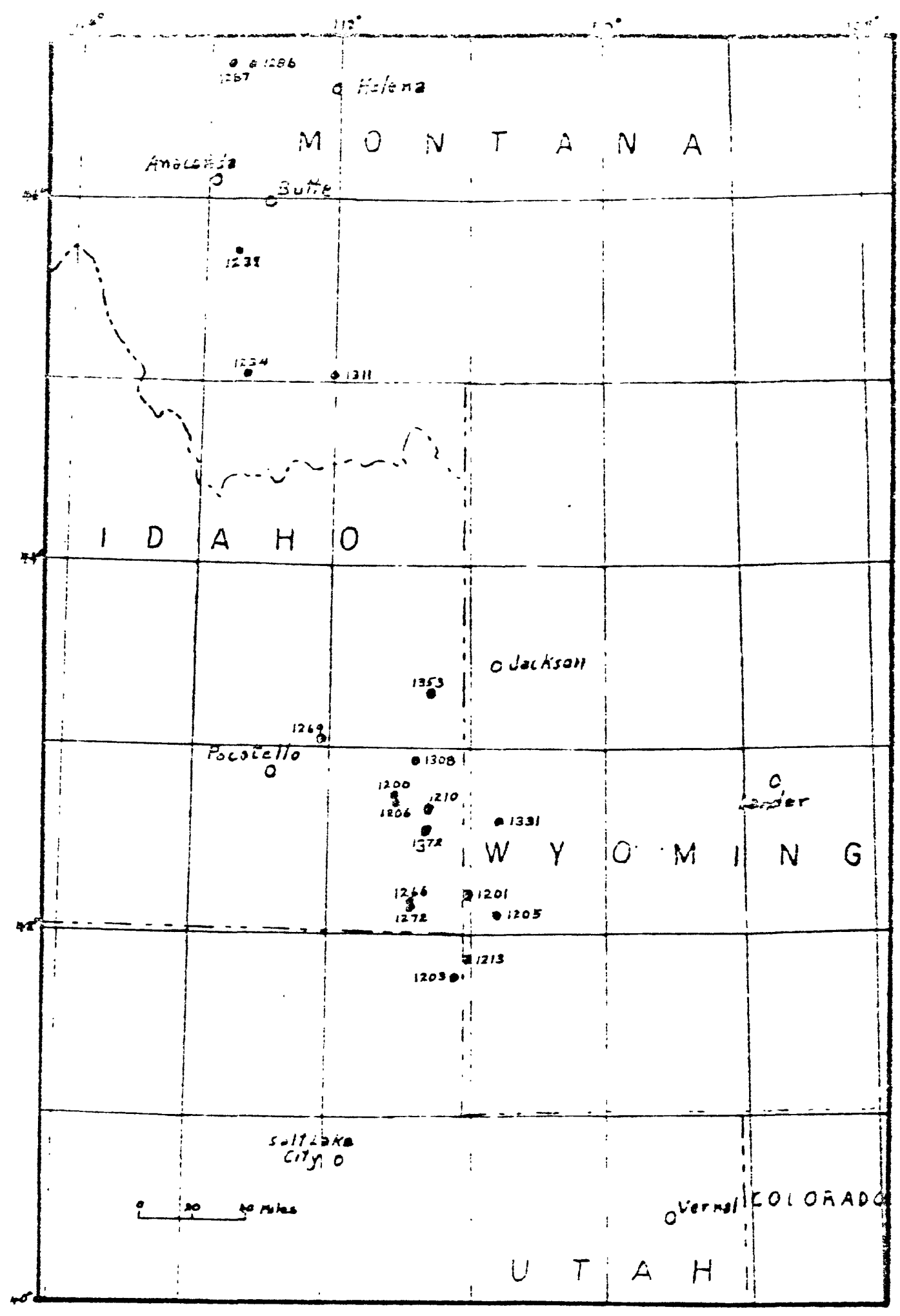

Figure 3.--Location of analyzed samples of tables 3 to 10 . Numbers are the lot numbers of the localities. 
Table 3. Mercury, thallium, and total nitrogen in samples of the Meade Peak Member of the Phosphoria Formation at Conda (lot 1200), Idahol

[Analyses by E. Campbe11]

\begin{tabular}{|c|c|c|c|c|c|}
\hline Bed no. & Sample no. & Rock type ${ }^{2}$ & $\begin{array}{r}\mathrm{Hg} \\
\text { ppm }\end{array}$ & $\begin{array}{l}\mathrm{T} 1 \\
\mathrm{ppm}\end{array}$ & $\begin{array}{l}\mathrm{N}(\text { total }) \\
\text { percent }\end{array}$ \\
\hline $\mathrm{P}-138$ & FCA-144 & $\mathbf{M}$ & 0.42 & 0.9 & 0.40 \\
\hline$P-134$ & FCA-135 & $M$ & .62 & 9.2 & .62 \\
\hline$P-131$ & FCA-132 & $\mathrm{P}$ & .49 & 2.3 & .46 \\
\hline $\mathrm{P}-125$ & $\mathrm{FCA}-126$ & $\mathrm{P}$ & .28 & 2.2 & .30 \\
\hline $\mathrm{P}-117$ & FCA-118 & M & .62 & 9.1 & .89 \\
\hline $\mathrm{P}-112$ & FCA-111 & $\mathrm{C}$ & .52 & 4.1 & .63 \\
\hline P-92 & FCA-102 & $\mathbf{M}$ & .31 & .7 & .54 \\
\hline$P-78$ & LES-232 & $\mathbf{M}$ & .34 & .9 & .40 \\
\hline $\mathrm{P}-72$ & LES-226 & $\mathrm{P}$ & .42 & .4 & .49 \\
\hline$P-65$ & LES-219 & $\mathbf{M}$ & .28 & .8 & .61 \\
\hline$P-54$ & FCA-88 & $\mathrm{C}$ & .27 & .7 & .61 \\
\hline$P-46$ & LES-212 & $M$ & .48 & .6 & .96 \\
\hline$P-42$ & LES-208 & $\mathbf{M}$ & .56 & .8 & .78 \\
\hline$P-20$ & $\mathrm{FCA}-74$ & $P$ & .44 & 3.4 & .31 \\
\hline$P-10$ & LES-200 & $P$ & .41 & 9.1 & .26 \\
\hline$P-4$ & LES-194 & M & .48 & 9.7 & .19 \\
\hline
\end{tabular}

${ }^{1}$ Stratigraphic section and other analyses in McKelvey, V. E., Davidson, D. F., O'Malley, F. W., and Smith, L. E., 1953, Stratigraphic Sections of the Phosphoria Formation in Idaho, 1947-48, Part 1: U.S. Geol. Survey Circ. 208, p. 25-33.

$$
{ }^{2} \mathrm{P}=\text { phosphate rock, } \mathrm{M}=\text { mudstone, and } \mathrm{C}=\text { carbonate rock. }
$$


Table 4. Mercury, thallium, and total nitrogen in samples of the Mead Peak Member of the Phosphoria Formation at Trail Canyon (1ot 1206), Idahol

[Analyses by E. Campbe11]

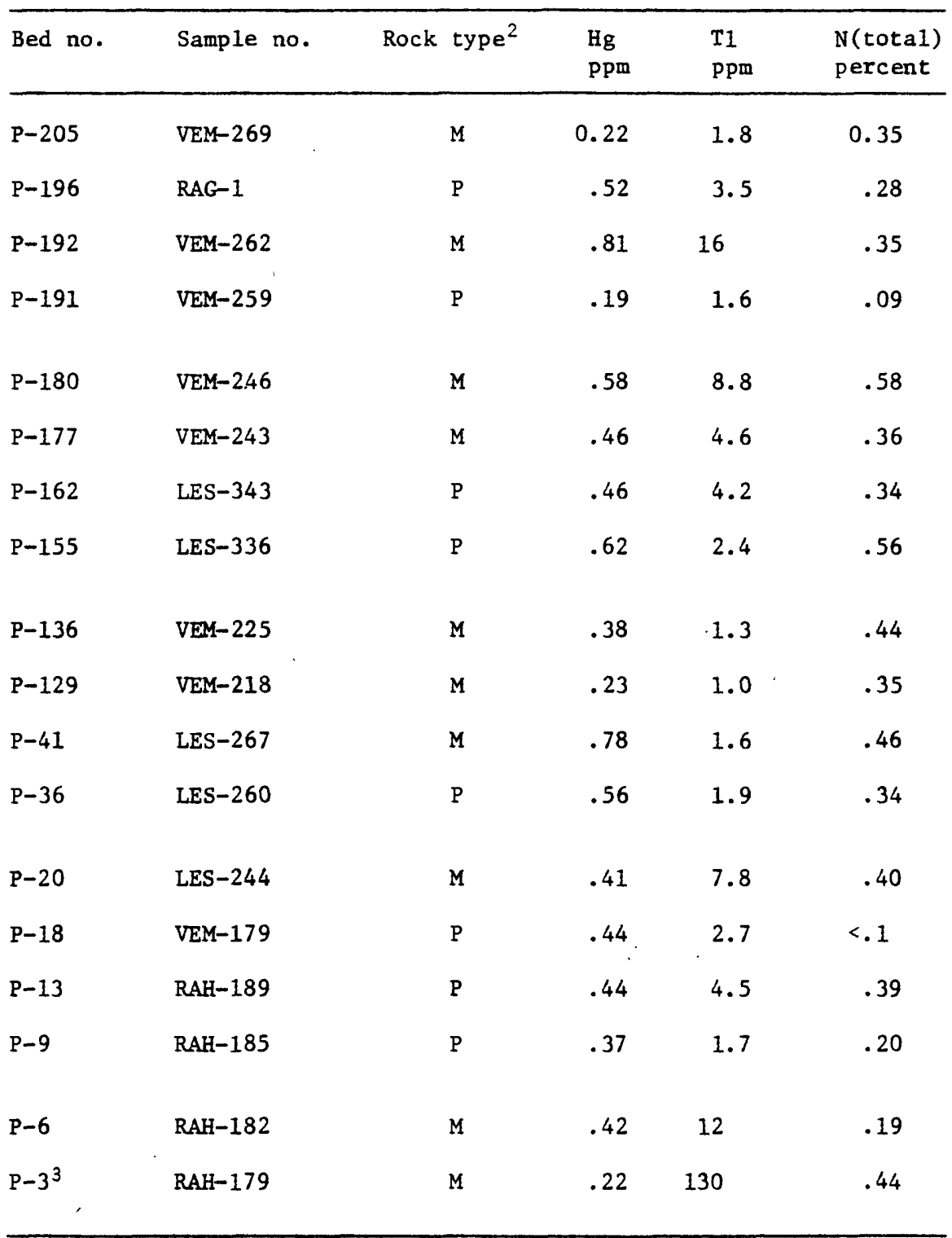

${ }^{1}$ Stratigraphic section and other analyses in McKelvey, V. E., Armstrong, F. C., Gulbrandsen, R. A., and Campbell, R. M., 1953, Stratigraphic Sections of the Phosphoria Formation in Idaho, 1947-48, Part 2: U.S. Geol. Survey Circ. 301, p. 21-31.

${ }^{2} \mathrm{P}=$ phosphate rock and $\mathrm{M}=$ mudstone.

${ }^{3}$ Sample also contains $4.9 \mathrm{ppm} \mathrm{Ag}, 210 \mathrm{ppm} \mathrm{B,} 850 \mathrm{ppm} \mathrm{Ba}, 3.7 \mathrm{ppm}$ Be, 590 ppm Cd, 150 ppm Co, 370 ppm Cr, 350 ppm Cu, 10 ppm Ga, 9 ppm Ge, 88 ppm La, 16,000 ppm Mn, 130 ppm Mo, >1,000 ppm N1, 16 ppm $\mathrm{Pb}, 14 \mathrm{ppm} \mathrm{Sc}, 480 \mathrm{ppm} \mathrm{Sr}, 1,000 \mathrm{ppm} \mathrm{V}, 99 \mathrm{ppm} \mathrm{Y,} \mathrm{13,000} \mathrm{ppm} \mathrm{Zn,} \mathrm{and}$ $340 \mathrm{ppm} \mathrm{Zr}$ (analyses by J. Fletcher). In addition, $3.5 \mathrm{ppm}$ Se was determined by J. S. Wahlberg, J. O. Johnson, and R. J. Young; and 280 ppm As was determined by E. J. Fennelly. 
Table 5. Chemical composition of samples from the vanadferous zone, Bloomington Canyon (10t 1272). Idaho'

[Emission spectrographic analysis by J. D. Fletcher for all constituents except those indicated here. Tl,

Hg, and N(total) analyzed by E. Campbell. Se analyzed by J. S. Wahlberg, J. O. Johnson, and R. J. Young.

As analyzed by R. J. Fennelly]

\begin{tabular}{|c|c|c|c|c|c|c|c|c|c|c|}
\hline Sample No. & RMC-827 & RMC-828 & RMC-829 & RMC-830 & RLP-832 & $R L P-833$ & RLP-835 & $\mathrm{DAB}-837$ & $\mathrm{DAB}-840$ & DAB-841 \\
\hline Lab. No. & 8986 & 8987 & 8988 & 8989 & 8991 & 8992 & 8994 & 8996 & 8999 & 9000 \\
\hline Element & $z$ & 7 & 7 & 7 & $\%$ & 7 & $\%$ & $\%$ & $\%$ & $\%$ \\
\hline S1 & 30 & 33 & $>34$ & 38 & 35 & 28 & 12 & 12 & 12 & 9.6 \\
\hline $\mathbf{A l}$ & 3.6 & 3.9 & 4.4 & 4.1 & 3.8 & 3.3 & 1.8 & 2.6 & 1.8 & 1.7 \\
\hline $\mathrm{Fe}$ & 1.3 & 2.1 & 1.4 & 1.5 & 2.5 & 2.4 & .68 & 2.4 & .61 & 1.2 \\
\hline Mg & .51 & .35 & .35 & .37 & .25 & .24 & .14 & .21 & .24 & .17 \\
\hline $\mathrm{Ca}$ & 3.5 & 3.2 & 1.2 & 1.0 & 1.1 & 4.3 & 5.6 & 19.5 & 7.9 & 6.5 \\
\hline Ma & .08 & .09 & .08 & .07 & $>.32$ & $>.32$ & .09 & $>.32$ & $>.32$ & .14 \\
\hline $\mathbf{K}$ & 1.4 & 1.4 & 1.4 & 1.7 & 1.4 & 1.2 & .39 & .60 & .55 & .49 \\
\hline T1 & .24 & .30 & .28 & .28 & .26 & .22 & .08 & .13 & .11 & .09 \\
\hline $\mathbf{P}$ & 2.1 & 2.9 & 1.6 & 1.2 & 1.4 & 4.0 & 6.2 & $>6.8$ & $>6.8$ & 7.0 \\
\hline \multirow[t]{2}{*}{$N($ total $)$} & .70 & 1.5 & 1.2 & 1.1 & 1.3 & 1.0 & 1.0 & .41 & 2.1 & 2.4 \\
\hline & ppm & ppr & pipn & ppm & ppm & ppm & ppm & $\mathrm{ppm}$ & $\mathrm{ppm}$ & ppm \\
\hline Ag & 16 & 16 & 14 & 14 & 12 & 14 & 16 & 14 & 22 & 22 \\
\hline As & 12 & 25 & 20 & 20 & 50 & 50 & 25 & 40 & 20 & 50 \\
\hline $\mathrm{Au}$ & $<10$ & $<10$ & $<10$ & $<10$ & $<10$ & $<10$ & $<10$ & $<10$ & $<10$ & $<10$ \\
\hline B & 190 & 200 & 190 & 190 & 140 & 130 & 25 & 68 & 140 & 49 \\
\hline $\mathrm{Ba}$ & 350 & 360 & 380 & 340 & 300 & 340 & 180 & 240 & 270 & 130 \\
\hline $\mathrm{Be}$ & 2.9 & 3.3 & 2.7 & 2.1 & 2.3 & 3.7 & 2.4 & 3.3 & 2.9 & 1.9 \\
\hline Bi & $<4.6$ & $<4.6$ & $<4.6$ & $<4.6$ & $<4.6$ & $<4.6$ & $<4.6$ & $<4.6$ & $<4.6$ & $<4.6$ \\
\hline Cd & 180 & 170 & 180 & 170 & 980 & 970 & 380 & 420 & 760 & 460 \\
\hline $\mathrm{Ce}$ & 130 & $<63$ & 69 & 73 & 110 & 130 & 99 & $<63$ & $<63$ & 150 \\
\hline Co & 1.4 & 2.1 & 3.5 & 2.6 & 10 & 9.5 & 1.0 & 2.2 & 2.3 & 2.8 \\
\hline Cr & 1,600 & 1,200 & 980 & 1,200 & 940 & 850 & 860 & 1,400 & 1,500 & 1,300 \\
\hline $\mathbf{C u}$ & 260 & 170 & 250 & 180 & 110 & 180 & 120 & 99 & 420 & 260 \\
\hline Dy & 7.8 & 14 & 8.7 & $<6.8$ & 17 & 24 & 9.8 & 19 & 14 & 14 \\
\hline $\mathbf{E r}$ & $<4.6$ & 8.7 & 6.6 & $<4.6$ & 11 & 27 & 7.9 & 10 & 9.3 & 12 \\
\hline Eu & 2.4 & 2.5 & 1.6 & 1.9 & 3.5 & 3.3 & 1.9 & 1.8 & 2.5 & 1.9 \\
\hline $\mathrm{Ga}$ & 7.9 & 8.2 & 9.1 & 9.2 & 8.9 & 7.2 & 3.8 & 5.6 & 9.8 & 5.1 \\
\hline Gd & $<15$ & 16 & 23 & 19 & 26 & 18 & $<4.6$ & $<15$ & 23 & $<4.6$ \\
\hline $\mathrm{Ge}$ & $<3.2$ & $<3.2$ & $<3.2$ & $<3.2$ & $<3.2$ & $<3.2$ & $<3.2$ & $<3.2$ & $<3.2$ & $<3.2$ \\
\hline Hf & $<22$ & $<22$ & $<22$ & $<22$ & $<22$ & $<22$ & $<22$ & $<22$ & $<22$ & $<22$ \\
\hline $\mathrm{Hg}$ & 1.1 & 1.6 & .80 & .86 & .78 & .80 & 1.3 & .60 & .94 & .91 \\
\hline
\end{tabular}


Table 5. Chemical composition of samples from the vanadiferous zone, Bloomington Canyon. (10t 1272), Idaho'--Cont.

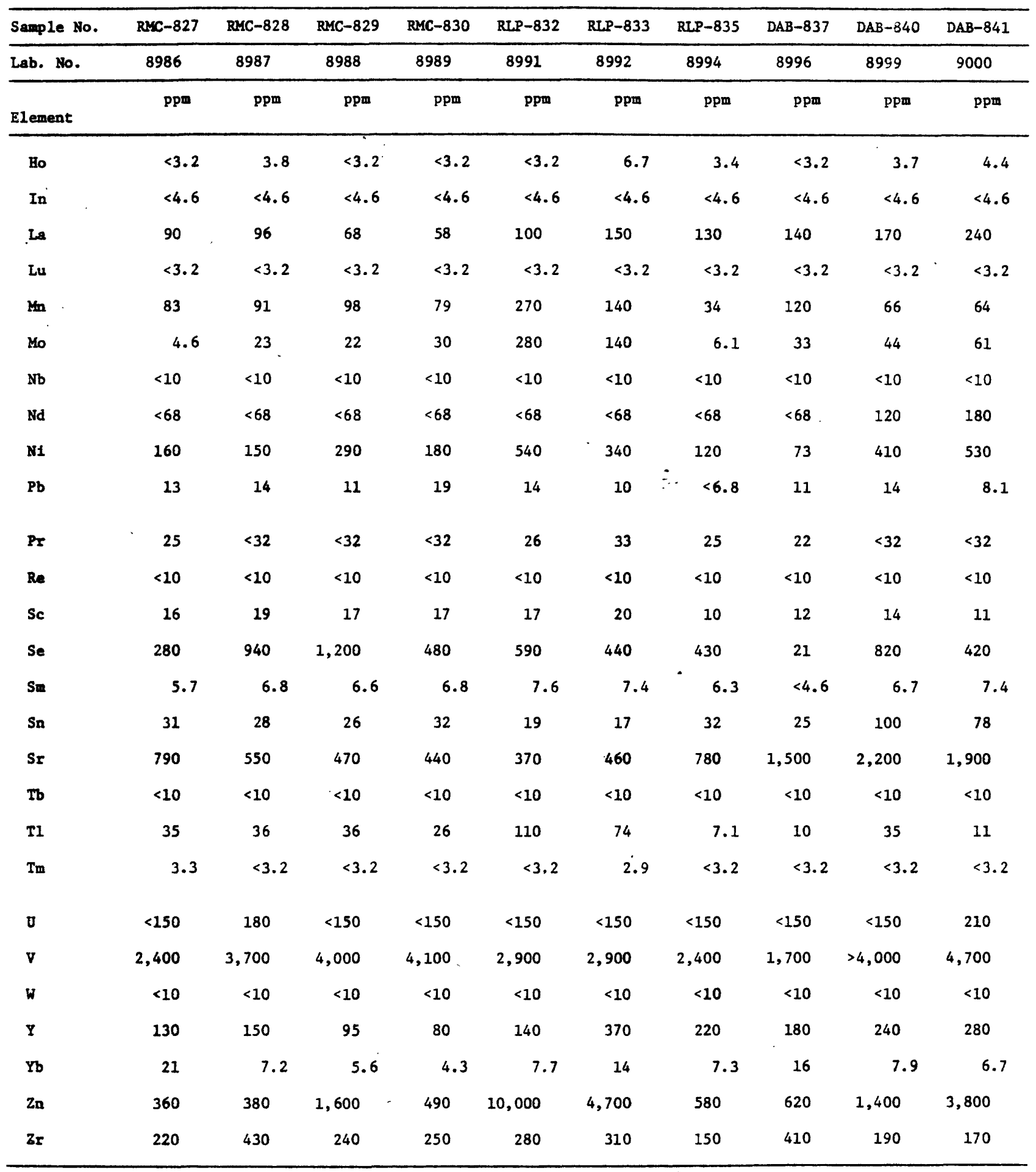

${ }^{1}$ Stratigraphic section in O'Malley, F. W., Davidson, D. F., Hoppin, R. A., and Sheldon, R. P., Stratigraphic Sections of the Phosphoria Formation in Idaho, 1947-48, Part 3: U.S. Geol. Survey C1rc. 262, p. 38-43. 
Table 6 . Analyses of two samples of the vanadiferous zone at Coa1 Canyon (lot 1201), Wyoming

[Semiquantitative spectrographic analyses by Harry Bastron]

(ppm)

\begin{tabular}{|c|c|c|}
\hline Bed no. & $P-73$ & $P-72$ \\
\hline Sample no. & VEM-42 & VEM -41 \\
\hline \multicolumn{3}{|l|}{ Element } \\
\hline $\mathrm{Ag}$ & 10 & 7 \\
\hline B & 100 & 70 \\
\hline $\mathrm{Ba}$ & 300 & 300 \\
\hline $\mathrm{Be}$ & 1 & -- \\
\hline Co & 7 & 7 \\
\hline $\mathrm{Cr}$ & 1,000 & 500 \\
\hline $\mathrm{Cu}$ & 100 & 70 \\
\hline $\mathrm{Ga}$ & 15 & 10 \\
\hline Mn & 150 & 200 \\
\hline Mo & 300 & 150 \\
\hline $\mathrm{Ni}$ & 700 & 200 \\
\hline $\mathrm{Pb}$ & 20 & 20. \\
\hline Sc & 15 & 15 \\
\hline Sr & 150 & 150 \\
\hline $\mathrm{v}$ & 7,000 & 5,000 \\
\hline $\mathrm{Y}$ & 30 & 50 \\
\hline $\mathrm{Yb}$ & 20 & 7 \\
\hline $\mathrm{Zn}$ & 15,000 & 700 \\
\hline Zr & 300 & 300 \\
\hline
\end{tabular}

${ }^{1}$ Stratigraphic section and other analyses in Gulbrandsen, 1960, Petrology of the Meade Peak Phosphat1c Shale Member of the Phosphoria Formation at Coal Canyon, Wyoming: U.S. Geol. Survey Bu11. 1111-C, 146 p. 
Table 7 . Vanadium analyses of the Phosphoria Formation at Bear Creek (lot 1353), Idaho 1

[Analyses by C. L. Waring and Katherine Vallentine] (percent by weight)

\begin{tabular}{|c|c|c|c|c|}
\hline Bed no. & Sample no. & & Rock type ${ }^{2}$ & v \\
\hline U-1 & MAW-657I & & $\mathrm{p}$ & 0.05 \\
\hline $\mathrm{P}-61$. & MAW-6567 & & $\mathrm{P}$ & 0.04 \\
\hline$P-60$ & MAW-6566 & & $\mathrm{P}$ & 0.008 \\
\hline$P-59$ & MAW-6565 & & $P$ & 0.05 \\
\hline $\mathrm{P}-58$ & MAW- 6564 & & P & 0.006 \\
\hline$P-57$ & MAW-6563 & & $\mathrm{P}$ & 0.004 \\
\hline$P-56$ & MAW-6562 & . & $\mathrm{P}$ & 0.006 \\
\hline $\mathrm{P}-55$ & MAW-656I & & $\mathrm{P}$ & 0.004 \\
\hline $\mathrm{P}-54$ & MAW- 6560 & & $\mathrm{P}$ & 0.004 \\
\hline $\mathrm{P}-53$ & MAW- 6559 & & $\mathrm{P}$ & 0.02 \\
\hline $\mathrm{P}-52$ & MAW-6558 & & $\mathrm{P}$ & 0.006 \\
\hline$P-5 I$ & MAW- 6557 & & $\mathbf{M}$ & 0.05 \\
\hline$P-50$ & MAW-6556 & & $\mathbf{M}$ & 0.06 \\
\hline$P-49$ & MAW-6555 & & $P \& M$ & 0.06 \\
\hline$P-48$ & MATW-6554 & & $\mathbf{M}$ & 0.09 \\
\hline$P-47$ & MAW-6553 & & $\mathbf{P}$ & 0.08 \\
\hline$P-46$ & MAW-6552 & & $\mathbf{M}$ & 0.35 \\
\hline$p-45$ & MAW-655I & & $\mathbf{M}$ & 0.40 \\
\hline$P-44$ & MAW-6550 & & $\mathbf{M}$ & 0.08 \\
\hline$P-43$ & RGW-6549 & & $\mathbf{M}$ & 0.04 \\
\hline$P-42$ & RGW-6548 & & $\mathbf{M}$ & 0.05 \\
\hline $\mathrm{P}-41$ & RGW-6547 & & $\mathbf{M}$ & 0.05 \\
\hline $\mathrm{p}-40$ & TMC -6546 & & $\mathbf{M}$ & 0.20 \\
\hline$P-39$ & TMC- 6545 & & $\mathbf{M}$ & 0.08 \\
\hline $\mathrm{P}-38$ & TMC -6544 & . & $\mathbf{M}$ & 0.08 \\
\hline $\mathrm{P}-37$ & TMC- 6543 & & $M$ & 0.06 \\
\hline $\mathrm{P}-36$ & TMC-6542 & & $\mathrm{M}$ & 0.09 \\
\hline $\mathrm{P}-35$ & TMC- 6541 & & M & 0.06 \\
\hline$P-34$ & TMC- 6540 & & M & 0.20 \\
\hline $\mathrm{P}-33$ & TMC-6539 & & $\mathbf{M}$ & 0.15 \\
\hline $\mathrm{p}-32$ & TMC- 6538 & & P\&M & 0.30 \\
\hline$P-31$ & TMC- 6537 & & $M$ & 0.20 \\
\hline$P-30$ & TMC- 6536 & & M\&P & 0.30 \\
\hline$P-29$ & TMC-6535 & & $\mathbf{P}$ & 0.35 \\
\hline $\mathrm{P}-28$ & TMC- 6534 & & $\mathbf{P}$ & 0.35 \\
\hline $\mathrm{P}-27$ & TMC -6533 & & $M$ & 0.25 \\
\hline $\mathrm{P}-26$ & TMC -6532 & & $\mathbf{M}$ & 0.20 \\
\hline $\mathrm{P}-25$ & TMC-6531 & & $M$ & 0.20 \\
\hline$P-24$ & TMC -6530 & & $\mathbf{M}$ & 0.09 \\
\hline $\mathrm{P}-23$ & TMC-6529 & & $\mathbf{M}$ & 0.20 \\
\hline
\end{tabular}


Table 7 . Vanadium analyses of the Phosphoria Formation at Bear Creek (1ot 1353), Idahol--Continued

\begin{tabular}{|c|c|c|c|}
\hline Bed no. & Sample no. & Rock type ${ }^{2}$ & $\mathrm{~V}$ \\
\hline $\mathrm{P}-22$ & TMC -6528 & M & 0.40 \\
\hline $\mathrm{P}-21$ & TMC -6527 & $M$ & 0.25 \\
\hline $\mathrm{P}-20$ & TMC- 6526 & $\mathrm{P}$ & 0.10 \\
\hline P-19 & TMC -6525 & $P$ & 0.20 \\
\hline $\mathrm{P}-18$ & RPS -6524 & $\mathbf{M}$ & 0.35 \\
\hline$P-17$ & RPS -6523 & $\mathrm{P}$ & 0.10 \\
\hline$P-16$ & RPS -6522 & $\mathrm{P}$ & 0.10 \\
\hline$P-15$ & RPS -6521 & $P$ & 0.07 \\
\hline$P-14$ & RPS -6520 & $P$ & 0.25 \\
\hline$P-13$ & RPS -6519 & $P$ & 0.25 \\
\hline$P-12$ & RPS -6518 & $P$ & 0.08 \\
\hline$P-11$ & RPS -6517 & $P$ & 0.06 \\
\hline$P-10$ & RPS -6516 & $\mathbf{M}$ & 0.25 \\
\hline$P-9$ & RPS- 6515 & $\mathbf{P}$ & 0.07 \\
\hline$P-8$ & RPS -6514 & $\mathbf{P}$ & 0.04 \\
\hline $\mathrm{p}-7$ & RPS -6513 & $\mathrm{P}$ & 0.08 \\
\hline$P-6$ & RPS-6512 & $P$ & 0.06 \\
\hline $\mathrm{P}-5$ & RPS- 6511 & $\mathbf{M}$ & 0.09 \\
\hline $\mathrm{P}-4$ & RPS -6510 & $\mathbf{P}$ & 0.05 \\
\hline$P-3$ & RPS -6509 & $\mathbf{M}$ & 0.20 \\
\hline $\mathrm{P}-2$ & RPS -6508 & $P$ & 0.04 \\
\hline$P-1$ & RPS -6570 & $\mathrm{C}$ & 0.04 \\
\hline$C w-23$ & TMC-6569 & Ch\&P & 0.04 \\
\hline$C w-17$ & TMC- 6568 & $\mathbf{M}$ & 0.03 \\
\hline
\end{tabular}

${ }^{1}$ Stratigraphic section and other analyses in Smart, R. A., Waring, R. G., Cheney, T. M., and Sheldon, R. P., 1954, Stratigraphic Sections of the Phosphoria Formation in Idaho, 1950-51: U.S. Geol. Survey Circ. 327 , p. 11-13.

${ }^{2} \mathrm{P}=$ phosphate rock, $\mathrm{M}=$ mudstone, $\mathrm{C}=$ carbonate rock, and $\mathrm{Ch}=$ chert. 
Table 8 . Vanadium analyses of the Phosphoria Formation at Gravel Creek Divide (lot 1308), Idaho ${ }^{1}$

[Analyses by C. L. Waring and Katherine Vallentine] (percent by weight)

\begin{tabular}{|c|c|c|c|}
\hline Bed no. & Sample no. & Rock type ${ }^{2}$ & $\mathrm{v}$ \\
\hline$P-74$ & RGW-5849 & M & 0.06 \\
\hline$P-73$ & RGW-5848 & M & 0.03 \\
\hline$P-72$ & RGW-5847 & $\mathbf{M}$ & 0.05 \\
\hline$P-71$ & RGW-5846 & M & 0.04 \\
\hline$P-70$ & RGW-5844 & M & 0.03 \\
\hline P-69 & RGW-5843 & $\mathbf{M}$ & 0.03 \\
\hline$P-68$ & RGW-5842 & M & 0.03 \\
\hline P-67 & RGW-5841 & M & 0.01 \\
\hline$P-66$ & RGW-5840 & $M$ & 0.07 \\
\hline $\mathrm{P}-65$ & RGW-5839 & $\mathrm{M} \& \mathrm{Ch}$ & 0.03 \\
\hline$P-64$ & RGW-5838 & Ch & 0.06 \\
\hline$P-63$ & RGW-5837 & $\mathbf{P}$ & 0.02 \\
\hline$P-62$ & HWP-5821 & M & 0.04 \\
\hline P-61 & HWP-5820 & M & 0.05 \\
\hline$P-60$ & HWP-5819 & M & 0.05 \\
\hline P-59 & HWP-5818 & $\mathbf{P}$ & 0.04 \\
\hline$P-58$ & HWP -5817 & $\mathbf{P}$ & 0.04 \\
\hline P-57 & HWP-5816 & $\mathbf{P}$ & 0.03 \\
\hline$P-56$ & HWP-5815 & $\mathbf{P}$ & 0.04 \\
\hline $\mathrm{P}-55$ & HWP-5814 & $\mathbf{P}$ & 0.03 \\
\hline$P-54$ & HWP-5813 & $\mathbf{P}$ & 0.09 \\
\hline P-53 & HWP-5812 & $\dot{\mathrm{P}}$ & 0.05 \\
\hline$P-52$ & HWP -5811 & M & 0.06 \\
\hline$P-51$ & HWP- 5810 & $\mathrm{c}$ & 0.02 \\
\hline$P-50$ & RAS -5870 & $\mathbf{P}$ & 0.02 \\
\hline$P-49$ & RAS-5869 & $\mathbf{P}$ & 0.06 \\
\hline$P-48$ & RAS-5868 & M & 0.04 \\
\hline$P-47$ & RAS-5867 & M & 0.04 \\
\hline$P-46$ & RAS-5866 & $\mathbf{P}$ & 0.02 \\
\hline$P-45$ & RAS-5865 & C & 0.005 \\
\hline$P-44$ & RAS-5864 & M & 0.02 \\
\hline$P-43$ & RAS-5863 & M & 0.04 \\
\hline$P-42$ & RAS-5862 & M & 0.02 \\
\hline$P-41$ & RAS-5861 & M & 0.05 \\
\hline$P-40$ & RAS -5860 & c & 0.01 \\
\hline P-39 & RAS-5859 & M & 0.04 \\
\hline $\mathrm{P}-38$ & RAS- 5858 & $P$ & 0.04 \\
\hline P-37 & RAS -5857 & M & 0.009 \\
\hline
\end{tabular}


Table 8 . Vanadium analyses of the Phosphoria Formation at Gravel Creek Divide (1ot (1308), Idaho--Continued

\begin{tabular}{|c|c|c|c|}
\hline Bed no. & Sample no. & Rock type ${ }^{2}$ & $\mathrm{v}$ \\
\hline $\mathrm{P}-36$ & RAS-5856 & $P$ & 0.02 \\
\hline $\mathrm{P}-35$ & RAS-5855 & $\mathbf{P}$ & 0.03 \\
\hline $\mathrm{P}-34$ & RAS-5854 & $\mathbf{M}$ & 0.09 \\
\hline $\mathrm{P}-33$ & RAS-5853 & $\mathbf{P}$ & 0.04 \\
\hline $\mathrm{P}-32$ & RAS-5852 & $M \& P$ & 0.07 \\
\hline$P-31$ & RAS-5851 & C & 0.04 \\
\hline $\mathrm{P}-30$ & HWP-5809 & $\mathbf{P}$ & 0.03 \\
\hline P-29 & HWP-5808 & $\mathbf{P}$ & 0.03 \\
\hline $\mathrm{P}-28$ & HWP-5807 & C & 0.005 \\
\hline $\mathrm{P}-27$ & HWP-5806 & C & 0.004 \\
\hline $\mathrm{P}-26$ & HWP-5805 & M & 0.05 \\
\hline $\mathrm{P}-25$ & HWP -5804 & P & 0.03 \\
\hline$P-24$ & HWP-5803 & M & 0.03 \\
\hline $\mathrm{P}-23$ & HWP-5802 & $\mathrm{P}$ & 0.04 \\
\hline $\mathrm{P}-22$ & HWP-5801 & $\mathrm{P}$ & 0.25 \\
\hline $\mathrm{P}-21$ & RGW-5836 & $\mathbf{P}$ & 0.09 \\
\hline $\mathrm{P}-20$ & RGW-5835 & P & 0.05 \\
\hline $\mathrm{P}-19$ & RGW-5834 & $P$ & 0.07 \\
\hline P-18 & RGW-5833 & $\mathbf{P}$ & 0.09 \\
\hline$P-17$ & RGW-5832 & $\mathrm{P}$ & 0.01 \\
\hline$P-16$ & RGW-5831 & $P$ & 0.05 \\
\hline $\mathrm{P}-15$ & RGW-5829 & M & 0.06 \\
\hline$P-14$ & RGW-5828 & $\mathbf{P}$ & 0.05 \\
\hline$P-13$ & RGW-5827 & $\mathrm{P}$ & 0.20 \\
\hline $\mathrm{P}-12$ & RGW-5826 & c & 0.05 \\
\hline $\mathrm{P}-11$ & HWP-5889 & $\mathbf{P}$ & 0.1 \\
\hline $\mathrm{P}-10$ & HWP-5888 & M & 0.1 \\
\hline P-9 & HWP-5887 & C & 0.06 \\
\hline$P-8$ & HWP-5886 & $P$ & 0.2 \\
\hline $\mathrm{P}-7$ & HWP-5885 & $P$ & 0.2 \\
\hline$P-6$ & MAW-5881 & $\mathbf{P}$ & 0.07 \\
\hline$P-5$ & MAW-5880 & $\mathbf{P}$ & 0.2 \\
\hline$P-4$ & MAW-5879 & $\mathbf{P}$ & 0.2 \\
\hline $\mathrm{P}-3$ & MAW-5878 & $\mathbf{P}$ & 0.2 \\
\hline $\mathrm{P}-2$ & MAW-5877 & C & 0.05 \\
\hline $\mathrm{P}-1$ & MAW-5876 & $\mathbf{P}$ & 0.02 \\
\hline
\end{tabular}

${ }^{1}$ Stratigraphic section and other analyses in Davidson, D. F., Smart, R. A., Peirce, H. W., and Weiser, J. D., 1953, Stratigraphic sections of the Phosphoria Formation in Idaho, 1949, Part 2: U.S. Geo1. Survey Circ. 305, p. 16-18.

${ }^{2} \mathrm{P}=$ phosphate rock, $\mathrm{M}=$ mudstone, $\mathrm{C}=$ carbonate rock, and $\mathrm{Ch}=$ chert. 
Table 9. Selenium analyses of parts of the Phosphoria Formation at 13 localities

[Analyses by J. H. McCarthy, J. L. Sierly, and E. J. Hackney] (ppm)

\begin{tabular}{|c|c|c|c|c|}
\hline Locality & Bed no. & Sample no. & Rock type & $\mathrm{Se}$ \\
\hline \multirow{16}{*}{$\begin{array}{l}\text { Conda, ID. } \\
\text { (1ot } 1200)\end{array}$} & $\mathrm{P}-133$ & FCA-134 & $P$ & 20 \\
\hline & $\mathrm{P}-132$ & $\mathrm{FCA}-133$ & $\mathbf{P}$ & 10 \\
\hline & $P-131$ & FCA-132 & $\mathbf{P}$ & 50 \\
\hline & $P-130$ & FCA-131 & $\mathrm{P}$ & $<10$ \\
\hline & $\mathrm{P}-129$ & FCA-130 & $\mathrm{P}$ & 20 \\
\hline & $\mathrm{P}-128$ & FCA-129 & $P$ & 30 \\
\hline & $\mathrm{P}-127$ & FCA-128 & $P$ & 20 \\
\hline & $P-126$ & FCA -127 & $\mathbf{P}$ & 40 \\
\hline & $\mathrm{P}-125$ & FCA-126 & $\mathrm{P}$ & 30 \\
\hline & $\mathrm{P}-15$ & LES-205 & $P$ & 30 \\
\hline & $\mathrm{P}-14$ & LES-204 & $\mathbf{P}$ & 30 \\
\hline & $\mathrm{P}-13$ & LES-203 & $\mathrm{p}$ & 20 \\
\hline & $\mathrm{P}-12$ & LES-202 & $P$ & 70 \\
\hline & $\mathrm{P}-11$ & LES-201 & $M$ & 100 \\
\hline & $\mathrm{P}-10$ & LES-200 & $\mathrm{P}$ & 50 \\
\hline & P-9 & LES-199 & $\mathbf{P}$ & 80 \\
\hline \multirow{6}{*}{$\begin{array}{l}\text { Mabie Canyon, ID. } 2 \\
\quad(\text { lot 1210) }\end{array}$} & $\mathrm{P}-11$ & RAW-37 & $\mathbf{P}$ & 70 \\
\hline & $P-10$ & RAW-36 & $P$ & 80 \\
\hline & P-9 & RAW-35 & $P$ & 200 \\
\hline & $\mathrm{P}-8$ & RAW-34 & $P$ & 100 \\
\hline & $\mathrm{P}-7$ & RAW-33 & $P$ & 100 \\
\hline & $P-6$ & RAW-32 & $P$ & 150 \\
\hline \multirow{14}{*}{$\begin{array}{l}\text { Paris Canyon, ID. }{ }^{3} \\
\quad(\text { lot 1266) }\end{array}$} & $\mathrm{P}-84$ & WOM-3142 & $\mathbf{M}$ & 20 \\
\hline & $P-83$ & พOM-3141 & M & 7 \\
\hline & $\mathrm{P}-82$ & WOM-3140 & $P$ & 15 \\
\hline & $P-81$ & WOM-3139 & $\mathbf{M}$ & 10 \\
\hline & $\mathrm{P}-80$ & WOM-3138 & $M$ & 15 \\
\hline & $\mathrm{P}-79$ & พOM-3137 & $P$ & 8 \\
\hline & $P-78$ & WOM-3136 & $M$ & 30 \\
\hline & $\mathrm{P}-76$ & พOM-3134 & $\mathbf{M}$ & 20 \\
\hline & $\mathrm{P}-75$ & WOM-3133 & M & 10 \\
\hline & $P-74$ & WOM-3132 & $M$ & 8 \\
\hline & $\mathrm{P}-73$ & WOM-3131 & $\mathbf{M}$ & 10 \\
\hline & $\mathrm{P}-72$ & WOM-3130 & $M$ & 9 \\
\hline & $\mathrm{P}-71$ & พOM-3129 & $\mathrm{p}$ & 10 \\
\hline & $P-70$ & WOM-3128 & $\mathbf{M}$ & 30 \\
\hline
\end{tabular}


Table 9. Selenium analyses of parts of the Phosphoria Formation at 13 localities--Continued

\begin{tabular}{|c|c|c|c|c|}
\hline Locality & Bed no. & Sample no. & Rock type ${ }^{l}$ & $\mathrm{Se}$ \\
\hline Fort Ha11, $\mathrm{ID}^{3}$ & $P-16$ & WOM-2148 & P & 7 \\
\hline \multirow[t]{8}{*}{ (lot 1269) } & $P-15$ & WOM-2147 & $P$ & 50 \\
\hline & $P-14$ & WOM-2146 & P & 50 \\
\hline & $P-13$ & WOM-2145 & $\mathbf{P}$ & 8 \\
\hline & $\mathrm{P}-12$ & WOM-2144 & $P$ & 8 \\
\hline & $P-7$ & WOM-2655 & $P$ & $<10$ \\
\hline & $P-6$ & WOM-2654 & $P$ & $<10$ \\
\hline & $P-4$ & WOM-2279 & $P$ & $<10$ \\
\hline & $\mathrm{P}-3$ & WOM-2278 & $\mathbf{P}$ & 4 \\
\hline Leefe, $\mathrm{WY}^{4}$ & $\mathrm{P}-16$ & พOM -3319 & $P \& M$ & 10 \\
\hline \multirow[t]{7}{*}{ (1ot 1213) } & $P-15$ & พOM-3318 & $P$ & 8 \\
\hline & $P-14$ & $\mathrm{RH}-3317$ & $\mathbf{P}$ & 8 \\
\hline & $P-13$ & $\mathrm{RH}-3316$ & $P$ & 5 \\
\hline & $\mathrm{P}-12$ & $\mathrm{RH}-3315$ & $P$ & 3 \\
\hline & $\mathrm{P}-11$ & WOM-3314 & $\mathbf{P}$ & 7 \\
\hline & $P-10$ & WOM-3313 & $\mathbf{P}$ & 20 \\
\hline & $P-9$ & WOM-3312 & $\mathrm{P}$ & 7 \\
\hline \multirow{8}{*}{$\begin{array}{l}\text { Middle Fork, } \\
\text { Pine Creek, } \mathrm{WY}^{4} \\
\text { (1ot 1205) }\end{array}$} & $P-58$ & LES-43 & M & 15 \\
\hline & $P-57$ & LES-44 & $\mathbf{P}$ & 10 \\
\hline & $P-56$ & LES-45 & M & 40 \\
\hline & $P-45$ & LES-68 & M & 60 \\
\hline & $P-44$ & FCA-17 & $\mathbf{M}$ & 80 \\
\hline & $P-43$ & FCA-18 & $\mathbf{M}$ & 80 \\
\hline & $P-42$ & FCA-19 & M & 10 \\
\hline & $P-41$ & FCA-20 & $\mathbf{M}$ & 60 \\
\hline \multirow{12}{*}{$\begin{array}{l}\text { Poison Creek, } \mathrm{WY}^{5} \\
\quad(\text { lot 1331) }\end{array}$} & $P-48$ & MAW-5130 & M & 15 \\
\hline & $P-47$ & MAW-5129 & M\&P & 80 \\
\hline & $P-46$ & MAW-5128 & $P \& M$ & 50 \\
\hline & $P-45$ & MAW-5127 & P & 50 \\
\hline & $P-44$ & MAW-5126 & $\mathbf{M}$ & 80 \\
\hline & $P-43$ & MAW-5125 & M & 100 \\
\hline & $P-42$ & MAW-5124 & M & 60 \\
\hline & $P-41$ & MAW-5123 & $\mathbf{M}$ & 50 \\
\hline & $P-40$ & MAW-5122 & M & 30 \\
\hline & $P-39$ & MAW-5121 & M & 40 \\
\hline & $P-38$ & MAW-5120 & M & 30 \\
\hline & $P-37$ & MAW-5119 & $\mathbf{M}$ & 30 \\
\hline \multirow{4}{*}{$\begin{array}{l}\text { Brazer Canyon, UT } \\
\quad(10 t \text { 1203) }\end{array}$} & $P-124$ & RAH-111 & $P$ & $<10$ \\
\hline & $\mathrm{P}-123$ & RAH-110 & $P$ & 10 \\
\hline & $\mathrm{P}-122$ & RAH-109 & $\mathbf{P}$ & 320 \\
\hline & $P-121$ & RAH-108 & P & $<5$ \\
\hline
\end{tabular}


Table 9. Selenium analyses of parts of the Phosphoria Formation at 13 localities--Continued

\begin{tabular}{|c|c|c|c|c|}
\hline Locality & Bed no. & Sample no. & Rock type $e^{1}$ & $\mathrm{Se}$ \\
\hline Sheep Creek, $\mathrm{MT}^{7}$ & $D-39$ & OAP-10 & $\mathbf{M}$ & 50 \\
\hline \multirow[t]{7}{*}{ (1ot 1234) } & $D-27$ & LAT- 22 & $M$ & 100 \\
\hline & $D-26$ & LAT -23 & $\mathbf{M}$ & 60 \\
\hline & $\mathrm{D}-25$ & LAT- 24 & $\mathbf{M}$ & 150 \\
\hline & $D-24$ & ERC-25 & M & 80 \\
\hline & $D-23$ & ERC-26 & $\mathbf{M}$ & 150 \\
\hline & $D-15$ & $D A B-34$ & $P \& M$ & 80 \\
\hline & $D-12$ & ERC-37 & $P \& M$ & 150 \\
\hline Canyon Camp, $\mathrm{MT}^{8}$ & $D-13$ & CWT-712 & $\mathbf{P}$ & 80 \\
\hline (lot 1311) & $D-6$ & CWT-705 & $P$ & 50 \\
\hline Melrose, $\mathrm{MT}^{9}$ & $D-10$ & MRK-293 & $P$ & 40 \\
\hline \multirow[t]{9}{*}{ (1ot 1239) } & $D-9$ & MRK-792 & $P \& M$ & 25 \\
\hline & $\mathrm{D}-8$ & MRK-291 & $\mathbf{P}$ & 20 \\
\hline & $D-7$ & MRK-290 & M & 100 \\
\hline & $D-6$ & MRK-289 & $\mathbf{P}$ & 8 \\
\hline & $D-5$ & MRK-288 & $\mathbf{M}$ & 80 \\
\hline & $D-4$ & MRK-287 & $\mathbf{P}$ & 40 \\
\hline & $D-3$ & MRK-286 & M\&P & 30 \\
\hline & $\mathrm{D}-2$ & MRK-285 & $\mathrm{P}$ & 20 \\
\hline & $D-1$ & MRK-284 & $P$ & 100 \\
\hline \multirow{6}{*}{$\begin{array}{l}\text { Anderson mine, } \mathrm{MT}^{9} \\
\quad(\text { ot 1287) }\end{array}$} & $D-7$ & MRK-313 & $P$ & 15 \\
\hline & $D-6$ & MRK-312 & $P$ & 8 \\
\hline & $D-5$ & MRK-311 & $\mathbf{P}$ & 5 \\
\hline & $D-4$ & MRK-310 & $P$ & 10 \\
\hline & $D-3$ & MRK-309 & $P$ & 8 \\
\hline & $D-2$ & MRK-308 & $\mathbf{P}$ & 25 \\
\hline \multirow{3}{*}{$\begin{array}{l}\text { Graveley mine, } \mathrm{MT}^{9} \\
\quad(\text { lot 1286) }\end{array}$} & $D-4$ & MRK-299 & $P$ & $<2$ \\
\hline & $D-3$ & MRK-298 & $P$ & 4 \\
\hline & $D-2$ & MRK-297 & $\mathbf{P}$ & 15 \\
\hline
\end{tabular}

${ }^{1} \mathrm{P}=$ Phosphate rock and $M=$ Mudstone.

${ }^{2}$ Stratigraphic section and other analyses in McKelvey, V. E., Davidson, D. F., O'Ma1ley, F. W., and Smith, L. E., 1953, Stratigraphic Sections of the Phosphoria Formation in Idaho, 1947-48, Part I: U.S. Geo1. Survey Circ. 208.

${ }^{3}$ Stratigraphic section and other analyses in $0^{\prime} \mathrm{Malley}$, F. W., Davidson, D. F., Hoppin, R. A., and Sheldon, R. P., 1953, Stratigraphic Sections of the Phosphoria Formation in Idaho, 1947-48, Part 3: U.S. Geo1. Survey Circ. 262. 
Table 9. Selenium analyses of parts of the Phosphoria Formation at 13 localities--Continued

${ }^{4}$ Stratigraphic section and other analyses in McKelvey, V. E., Smith, L. E., Hoppin, R. A., and Armstrong, F. C., 1953, Stratigraphic Sections of the Phosphoria Formation in Wyoming, 1947-48: U.S. Geol. Survey Circ. 210.

${ }^{5}$ Stratigraphic section and other analyses in Sheldon, R. P., Waring, R. G., Warner, M. A., and Smart, R. A., 1953, Stratigraphic Sections of the Phosphoria Formation in Wyoming, 1949-50: U.S. Geol. Survey Circ. 307.

${ }^{6}$ Stratigraphic section and other analyses in Smith, L. E., Hosford, G. F., Sears, R. S., Sprouse, D. P., and Stewart, M. D., 1952, Stratigraphic Sections of the Phosphoria Formation in Utah, 1947-48:

U.S. Geol. Survey Circ. 211.

${ }^{7}$ Stratigraphic section and other analyses in Swanson, R. W., Lowe11, W. R., Cressman, E. R., and Bostwick, D. A., 1953, Stratigraphic Sections of the Phosphorfa Formation in Montana, 1947-48: U.S. Geol. Survey Circ. 209.

${ }^{8}$ Stratigraphic section and other analyses in Swanson, R. W., Cressman, E. R., Jones, R. S., and Replogle, B. K., 1953, Stratigraphic Sections of the Phosphoria Formation in Montana, Part 2, 1949-50:

U.S. Geol. Survey Circ. 303.

${ }^{9}$ Stratigraphic section and other analyses in Klepper, M. R., Honkala, F. S., Payne, O. A., and Ruppel, E. T.; 1953, Stratigraphic Sections of the Phosphoria Formation in Montana, 1948: U.S. Geol. Survey Circ. 260. 


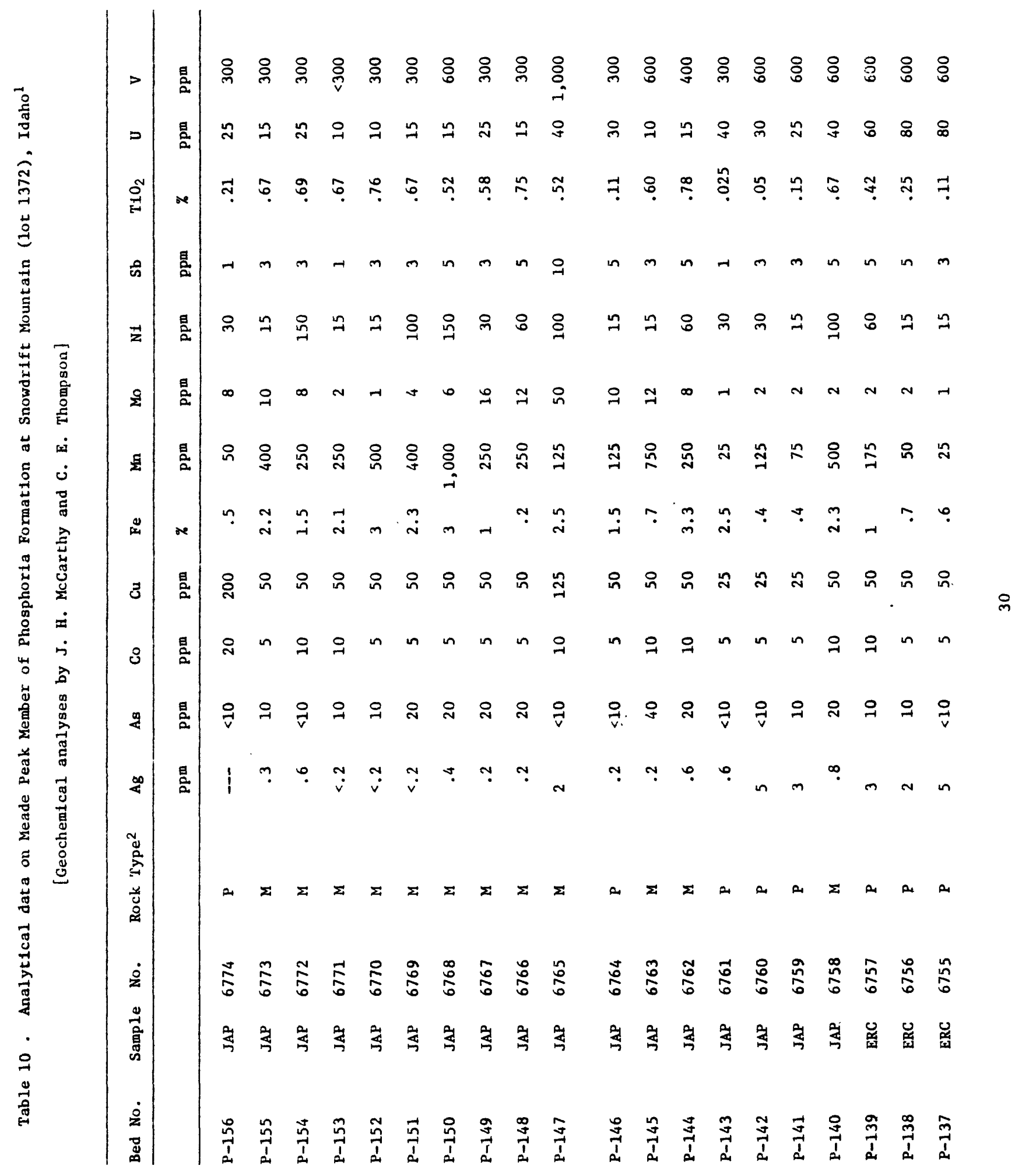




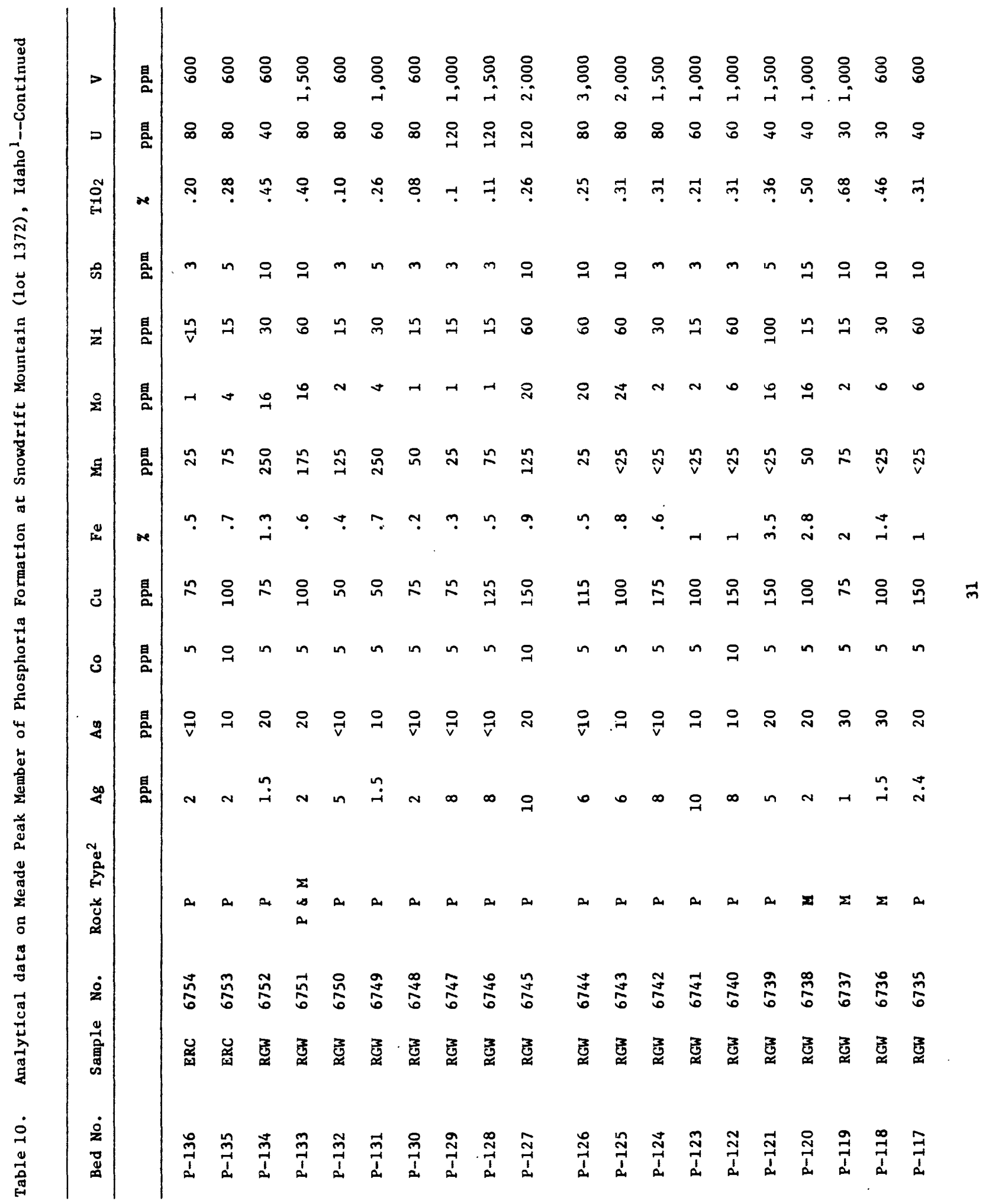




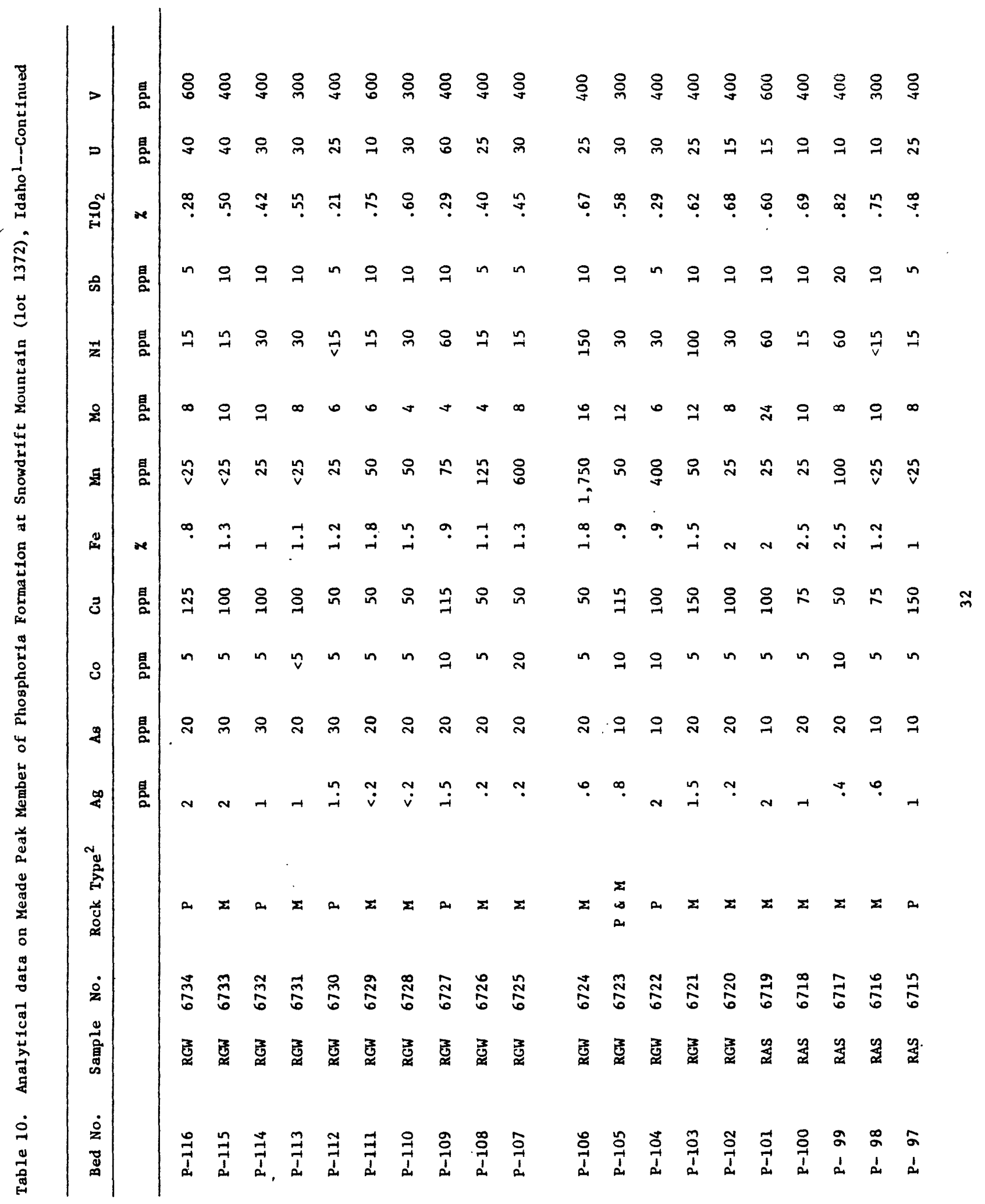




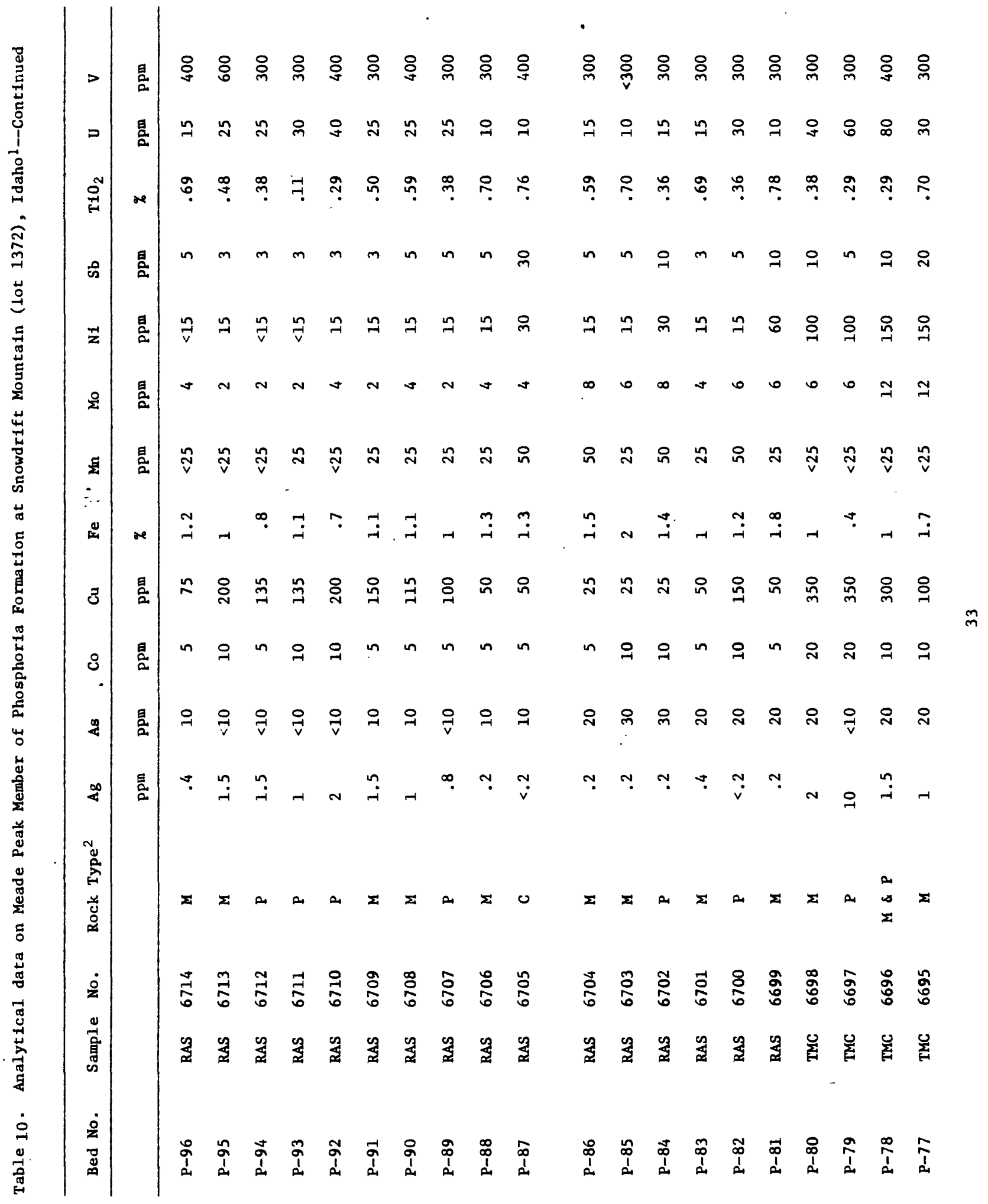




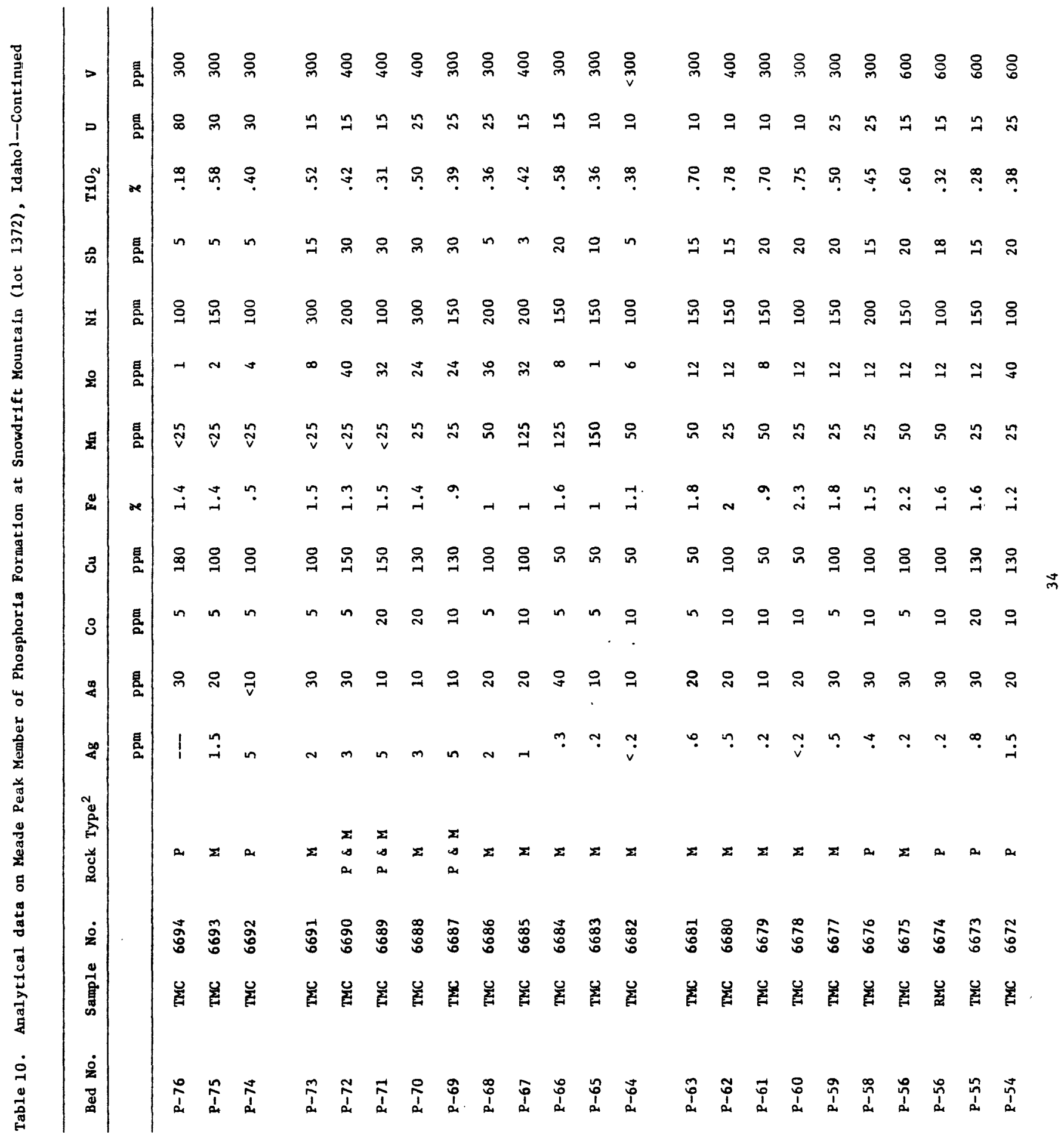




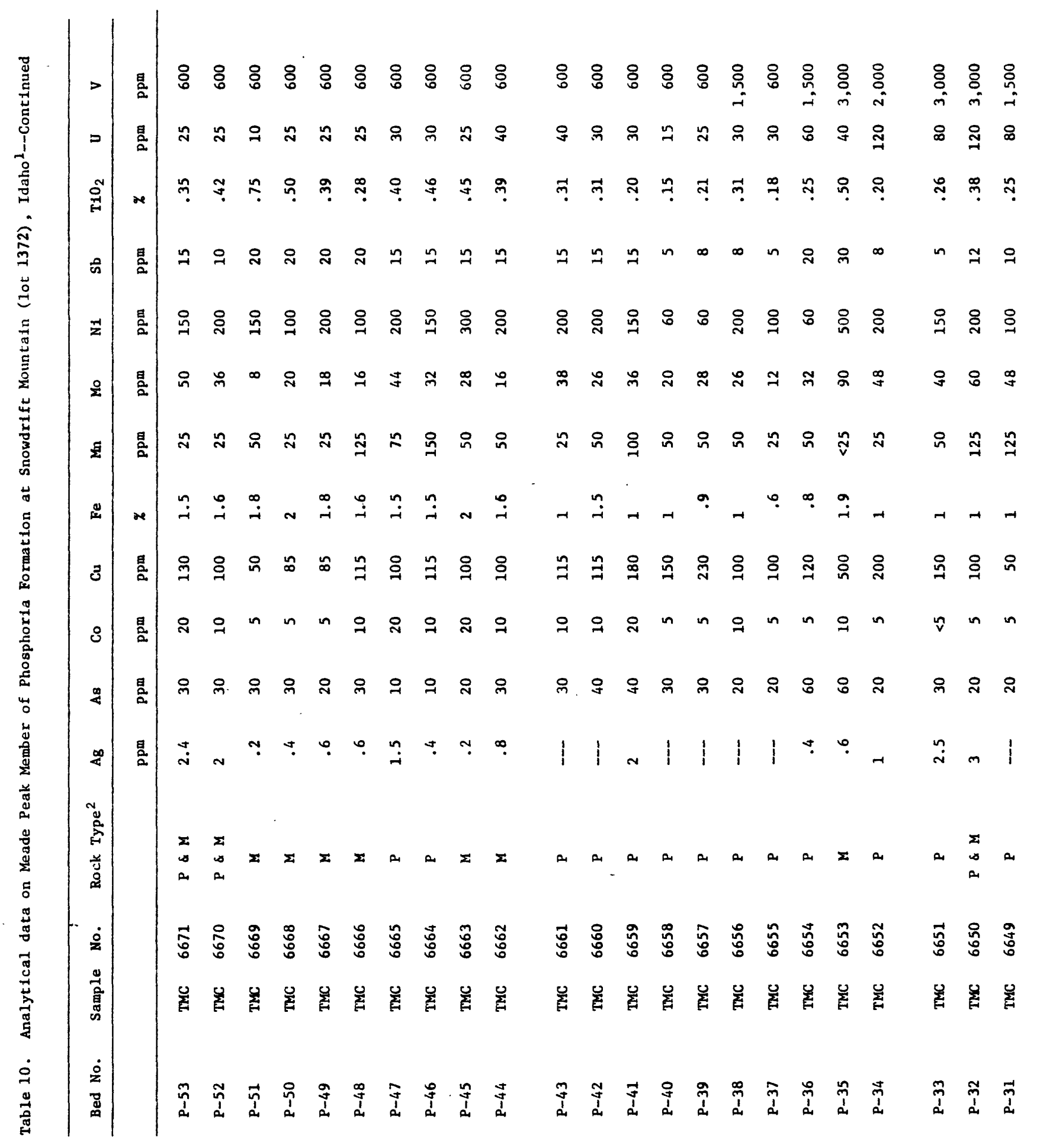




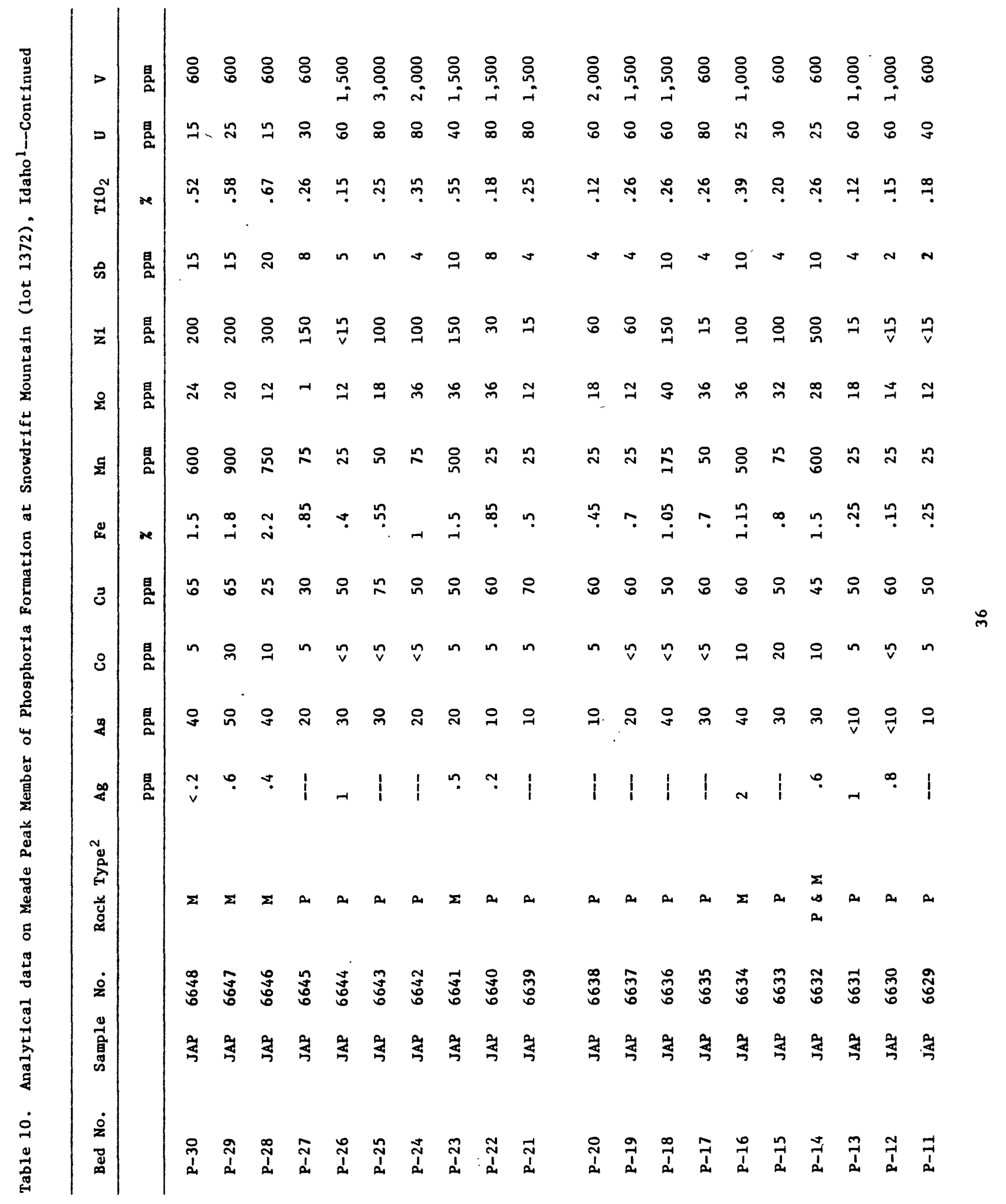




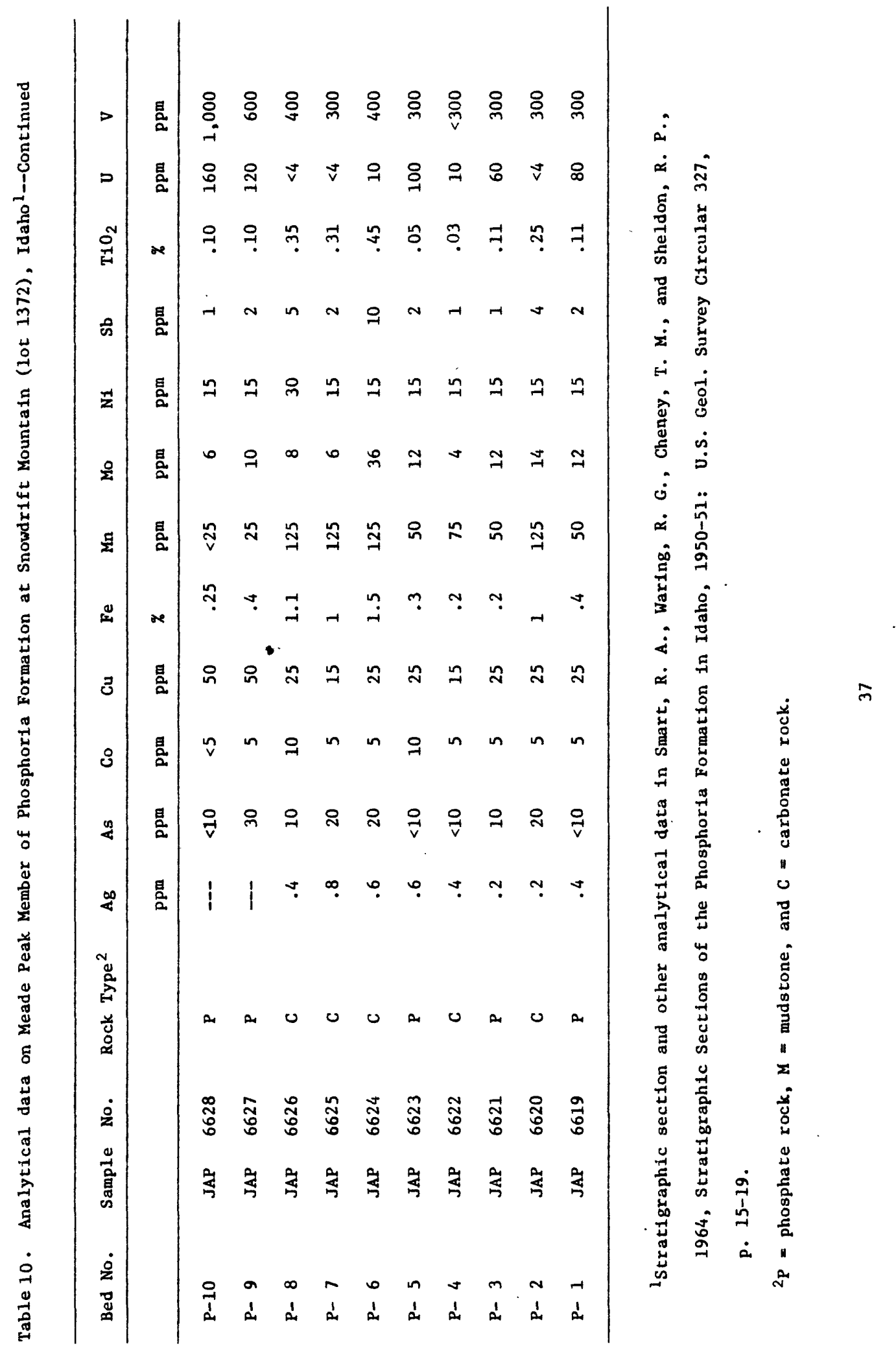




\section{References}

\section{U.S. Geological Survey Professional Papers}

Altschuler, Z. S., Cuttitta, F., and Berman, S., 1967, Rare earths in phosphorites - geochemistry and economic potential: U.S. Geol. Survey Prof. Paper 575-B, P. B1-B9.

Cressman, E. R., and Swanson, R. W., 1964, Stratigraphy and petrology of the Permian rocks of southwestern Montana: U.S. Geol. Survey Prof. Paper 313-C, p. C275-C569

Davidson, D. F., and Lakin, H. W., 1961, Metal content of some black shales of the western United States: U.S. Geol. Survey Prof. Paper 424-C, p. C329-C331

Gulbrandsen, R. A., 1970, Relation of carbon dioxide content of apatite of the Phosphoria Formation to regional facies: U.S. Geol. Survey Prof. Paper 700-B, p. B9-B13.

Gulbrandsen, R. A., and Reeser', D. W., 1969, An occurrence of Permian manganese nodules near Dillon, Montana: U.S. Geol. Survey Prof. Paper 650-C, p. C49-C57.

Love, J. D., 1961, Vanadium and associated elements in the Phosphoria Formation in the Afton area, western Wyoming: U.S. Geol. Survey Prof. Paper 424-C, p. C279-C282.

McKelvey, V. E., Williams, J. Steele, Sheldon, R. P., Cressman, E. R., Cheney, T. M., and Swanson, R. S., 1959, The Phosphoria, Park City, and Shedhorn Formations in the western phosphate field: U.S. Geol. Survey Prof. Paper 313-A, p. Al-A47. 
Murata, K. J., Friedman, Irving, and Gulbrandsen, R. A., 1972, Geochemistry of carbonate rocks in Phosphoria and related formations of the western phosphate field: U.S. Geol. Survey Prof. Paper 800-D, p. D103-D110. Sheldon, R. P., 1963, Physical stratigraphy and mineral resources of Permian rocks in western Wyoming: U.S. Geol. Survey Prof. Paper $313-B$, P. B49-B273.

Swanson, R. W., 1970, Mineral resources in Permian rocks of southwest Montana: U.S. Geo1. Survey Prof. Paper 313-E, p. E661-E777. Swanson, R. W., 1973, Geology and phosphate deposits of the Permian rocks in central western Montana: U.S. Geol. Survey Prof. Paper 313-F, p. F779-F833.

\section{U.S. Geological Survey Bulletins}

Gulbrandsen, R. A., 1960, Petrology of the Meade Peak Phosphatic Shale Member of the Phosphoria Formation at Coal Canyon, Wyoming: U.S. Geo1. Survey Bul1. 1111-C, p. C71-C146

Lotspeich, F. B., and Markward, E. L., 1963, Minor elements in bedrock, soil, and vegetation at an outcrop of the Phosphoria Formation on Snowdrift Mountain, southeastern Idaho: U.S. Geol. Survey Bull. 1181-F, p. F1-F42.

Montgomery, K. M., and Cheney, T. M., 1967, Geology of the Stewart Flat quadrangle, Caribou County, Idaho: U.S. Geol. Survey Bull. 1217, p. 1-63.

Thompson, M. E., 1953, Distribution of uranium in rich phosphate beds of the Phosphoria Formation: U.S. Geol. Survey Bu11. 988-D, p. D45-D67. Thompson, M. E., 1954, Further studies of the distribution of uranium in rich phosphate beds of the Phosphoria Formation: U.S. Geol. Survey Bu11. 1009-D, p. D107-D123. 
Vine, J. D., 1969, Element distribution in some Paleozoic black shales and associated rocks: U.S. Geol. Survey Bull. 1214-G, P. GI-G32.

U.S. Geological Survey Journal of Research

Gulbrandsen, R. A., 1974, Buddingtonite, ammonium feldspar, in the

Phosphoria Formation, southeastern Idaho: U.S. Geol. Survey Jour. Research, v. 2, no. 6, p. 693-697.

U.S. Geological Survey Circulars

Cheney, T. M., Sheldon, R. P., Waring, R. G., and Warner, M. A., 1954,

Stratigraphic sections of the Phosphoria Formation in Wyoming, 1951:

U.S. Geol. Survey Circ. 324, 22 p.

Cheney, T. M., Smart, R. A., Waring, R. G., and Warner, M. A., 1953,

Stratigraphic sections of the Phosphoria Formation in Utah,

1949-51: U.S. Geol. Survey Circ. 306, 40 p.

Cressman, E. R., Wilson, W. H., Tandy, C. W., and Garmoe, W. J., 1953,

Stratigraphic sections of the Phosphoria Formation in Montana,

1949-50, pt. 1, U.S. Geo1. Survey Circ. 302, 23 p.

Davidson, D. F., Smart, R. A., Peirce, H. W., and Weiser, J. D., 1953,

Stratigraphic sections of the Phosphorla Formation in Idaho, 1949,

pt. 2: U.S. Geol. Survey Circ. 305, 28 p.

Klepper, M. R., Honkala, F. S., Payne, 0. A., and Ruppel, E. T., 1953,

Stratigraphic sections of the Phosphoria Formation in Montana,

1948: U.S. Geol. Survey Circ. 260, 39 p.

McKelvey, V. E., and others, 1953, Stratigraphic sections of the

Phosphoria Formation in Wyoming, 1947-48: U.S. Geol. Survey Circ. $210,35 \mathrm{p}$. 
McKelvey, V. E., Armstrong, F. C., Gulbrandsen, R. A., and Campbell, R. M., 1953, Stratigraphic sections of the Phosphoria Formation in Idaho, 1947-48, pt. 2: U.S. Geol. Survey Circ. 301, 58 p. McKelvey, V. E., Davidson, D. F., O'Malley, F. W., and Smith, L. E., 1953, Stratigraphic sections of the Phosphoria Formation in Idaho, 1947-48, pt. 1: U.S. Geol. Survey Circ. 208, 49 p. 0'Malley, F. W., Davidson, D. F., Hoppin, R. A., and Sheldon, R. P., 1953, Stratigraphic sections of the Phosphoria Formation in Idaho, 1947-48, pt. 3: U.S. Geol. Survey Circ. 262, 43 p.

Peterson, J. A., Gosman, R. F., and Swanson, R. W., 1954, Stratigraphic sections of the Phosphoria Formation in Montana, 1951: U.S. Geol. Survey Circ. 326, 27 p.

Sche11, E. M., and Moore, K. P., 1970, Stratigraphic sections and chemical analyses of phosphatic rocks of Permian and Mississippian age in Weber County, Utah: U.S. Geo1. Survey Circ. 635, p. 1-11.

Sheldon, R. P., Cressman, E. R., Carswell, L. D., and Smart, R. A., 1954, Stratigraphic sections of the Phosphoria Formation in Wyoming, 1952: U.S. Geol. Survey Circ. 325, 24 p.

Sheldon, R. P., Waring, R. G., Warner, M. A., and Smart, R. A., 1953, Stratigraphic sections of the Phosphoria Formation in Wyoming, 1949-50: U.S. Geo1. Survey Circ. 307, 45 p.

Sheldon, R. P., Warner, M. A., Thompson, M. E., and Peirce, H. W., 1953, Stratigraphic sections of the Phosphoria Formation in Idaho, 1949, pt. 1: U.S. Geol. Survey Circ. 304,30 p. 
Smart, R. A., Waring, R. G., Cheney, T. M., and Sheldon, R. P., 1954, Stratigraphic sections of the Phosphoria Formation in Idaho, 1950-51: U.S. Geol. Survey Circ. 327, 22 p.

Smith, L. E., Hosford, G. F., Sears, R. S., Sprouse, D. P., and Stewart, M. D., 1952, Stratigraphic sections of the Phosphoria Formation in Utah, 1947-48: U.S. Geol. Survey Circ. 211, 48 p.

Swanson, R. W., Carswell, L. D., Sheldon, R. P., and Cheney, T. M., 1956, Stratigraphic sections of the Phosphoria Formation, 1953: U.S. Geo1. Survey Circ. 375, 30 p. Swanson, R. W., Cressman, E. R., Jones, R. S., and Replogle, B. K., 1953, Stratigraphic sections of the Phosphoria Formation in Montana, 1949-50, pt. 2: U.S. Geol. Survey Circ. 303, 21 p.

Swanson, R. W., Lowell, W. R., Cressman, E. R., and Bostwick, D. A., 1953, Stratigraphic sections of the Phosphoria Formation in Montana, 1947-48: U.S. Geol. Survey Circ. 209, 31 p.

U.S. Geological Survey Trace Element Investigation Reports (contains uranium analyses in addition to data included in the above Circulars)

Cheney, T. M., Sheldon, R. P., Waring, R. G., and Warner, M. A., November 1953, Stratigraphic sections of the Phosphoria Formation in Wyoming, 1951: U.S. Geo1. Survey TEI 377.

Cheney, T. M., Smart, R. A., Waring, R. G., and Warner, M. A., July 1953, Stratigraphic sections of the Phosphoria Formation in Utah, 1949-51: U.S. Geol. Survey TEI 360 .

Cressman, E. R., Wilson, W. H., Tandy, C. W., and Garmoe, W. J., July 1953, Stratigraphic sections of the Phosphoria Formation in Montana, 1949-50, pt. 1: U.S. Geol. Survey TEI 361. 